\title{
Eigenvalues of elliptic operators and geometric applications
}

\author{
Alexander Grigor'yan, Yuri Netrusov, and Shing-Tung Yau
}

\section{Contents}

1. Introduction

2. Energy forms on measure spaces

3. Decomposition of a pseudometric space by annuli 169

4. Estimating the counting function of an energy form 180

5. Eigenvalues on Riemannian manifolds 190

6. Eigenvalues of the Jacobi operator 200

$\begin{array}{ll}\text { References } & 214\end{array}$

\section{Introduction}

Eigenvalues and capacitors. Let $X$ be a Riemannian manifold and $\Delta$ be the Laplace operator on $X$. It is well-known that if $X$ is compact then the spectrum of $-\Delta$ is discrete and consists of an increasing sequence $\left\{\lambda_{k}\right\}_{k=1}^{\infty}$ of the eigenvalues (counted with the multiplicities) where $\lambda_{1}=0$ and $\lambda_{k} \rightarrow \infty$ as $k \rightarrow \infty$. Moreover, if $n=\operatorname{dim} X$ then Weyl's asymptotic formula says that

$$
\lambda_{k} \sim c_{n}\left(\frac{k}{\mu(X)}\right)^{2 / n}, \quad k \rightarrow \infty,
$$

where $\mu$ is the Riemannian measure on $X$ and $c_{n}>0$ is a constant depending only on $n$.

In this paper we develop a method of obtaining upper bounds for eigenvalues via capacities. A capacitor on $X$ is a couple $(F, G)$ of Borel sets in $X$ such that $F \subset G$. The capacity of the capacitor $(F, G)$ is defined by

$$
\operatorname{cap}(F, G)=\inf _{\varphi \in \mathcal{T}} \int_{X}|\nabla \varphi|^{2} d \mu
$$

where $\mathcal{T}=\mathcal{T}(F, G)$ is the class of test function, which consists of all functions $\varphi \in C_{0}^{\infty}(X)$ such that $\operatorname{supp} \varphi \subset \stackrel{o}{G}$ and $\varphi \equiv 1$ in a neighborhood of $F$.

The second author was supported by an EPSRC Advanced Research Fellowship AF/97/1657.

The third author was supported in part by NSF Grant No. DMS 95-04834. 
Assume that there exist $k$ disjoint capacitors $\left(F_{i}, G_{i}\right)$ in $X$ (that is, the sets $G_{i}$ are disjoint) satisfying the following properties, for all $i=1,2, \ldots, k$, and for some positive constants $v$ and $\varkappa$ :

(a) $\mu\left(F_{i}\right) \geq v$;

(b) $\operatorname{cap}\left(F_{i}, G_{i}\right) \leq \varkappa$.

Then, for any $\varepsilon>0$, there exists a test function $\varphi_{i}$ of the capacitor $\left(F_{i}, G_{i}\right)$ such that

$$
\int_{X}\left|\nabla \varphi_{i}\right|^{2} d \mu \leq \varkappa+\varepsilon
$$

The condition $(a)$ implies

$$
\int_{X} \varphi_{i}^{2} d \mu \geq \mu\left(F_{i}\right) \geq v
$$

whence

$$
\int_{X}\left|\nabla \varphi_{i}\right|^{2} d \mu \leq \Lambda \int_{X} \varphi_{i}^{2} d \mu
$$

where $\Lambda=\frac{\varkappa+\varepsilon}{v}$. Since the supports of the functions $\varphi_{i}$ are disjoint, it follows that

$$
\int_{X}|\nabla \varphi|^{2} d \mu \leq \Lambda \int_{X} \varphi^{2} d \mu
$$

for any $\varphi \in \mathcal{V}:=\operatorname{span}\left(\varphi_{1}, \ldots, \varphi_{k}\right)$. Note that $\mathcal{V}$ is a $k$-dimensional subspace of $C_{0}^{\infty}(X)$. The fact that any function $\varphi \in \mathcal{V}$ satisfies (1.3) implies that $\lambda_{k} \leq \Lambda$. Since this is true for any $\varepsilon>0$, we obtain that the hypotheses $(a)$ and $(b)$ imply that

$$
\lambda_{k} \leq \frac{\varkappa}{v}
$$

Clearly, $(a)$ implies $v \leq \frac{\mu(X)}{k}$. Under certain conditions, one can hope to get $k$ disjoint capacitors satisfying $(a)$ and $(b)$ with $v=c \frac{\mu(X)}{k}$, where $c$ is a small enough positive constant. In this case one obtains the following upper bound for $\lambda_{k}$ :

$$
\lambda_{k} \leq \frac{\varkappa}{c} \frac{k}{\mu(X)} .
$$

Comparison with (1.1) shows that if $\varkappa$ is independent of $k$ and the dimension $n$ is equal to 2 then the estimate (1.4) is sharp up to a constant factor.

This approach was successfully used by Korevaar [37] in the proof of the following result. Let $X=\left(\Sigma_{\gamma}, g\right)$ where $\Sigma_{\gamma}$ is the oriented compact Riemann surface of genus $\gamma$ and $g$ is an arbitrary Riemannian metric on $\Sigma_{\gamma}$. Then the eigenvalues of the Laplace operator on $X$ admit for all $k \geq 1$ the estimate ${ }^{1}$

$$
\lambda_{k} \leq C(\gamma+1) \frac{k}{\mu(X)},
$$

where $C$ is an absolute constant. Note that the metric $g$ is involved in the estimate (1.5) only through the total volume $\mu(X)$.

${ }^{1}$ Since $\lambda_{1}=0$, by changing the constant $C$ in (1.5) one obtains

$$
\lambda_{k} \leq C(\gamma+1) \frac{k-1}{\mu(X)} .
$$

The same applies to estimates (1.14 and (1.16) below. However, the distinction between $k$ and $k-1$ is marginal for our purpose. 
For $\lambda_{2}$, Yang and the third author [56] proved earlier a sharper estimate ${ }^{2}$

$$
\lambda_{2} \leq \frac{8 \pi(\gamma+1)}{\mu(X)} .
$$

The estimate (1.5) was stated in [57] as a conjecture, which was eventually settled by Korevaar.

Both works [37] and [56] have used the fact that there is a conformal mapping $T: X \rightarrow \mathbb{S}^{2}$ of degree at most $\gamma+1$. Let $\mu^{*}$ be the measure on $\mathbb{S}^{2}$, which is the $T$-pullback of the measure $\mu$ on $X$, and let cap* be the capacity on $\mathbb{S}^{2}$ associated with the standard Riemannian metric of $\mathbb{S}^{2}$. Assume that there exist $k$ disjoint capacitors $\left(F_{i}^{*}, G_{i}^{*}\right)$ on $\mathbb{S}^{2}$ satisfying the following conditions, for all $i=1,2, \ldots, k$ :

$$
\left(a^{\prime}\right) \mu^{*}\left(F_{i}^{*}\right) \geq v:=c \frac{\mu^{*}\left(\mathbb{S}^{2}\right)}{k} ;
$$

$\left(b^{\prime}\right) \operatorname{cap}^{*}\left(F_{i}^{*}, G_{i}^{*}\right) \leq C$,

where $c$ and $C$ are positive absolute constants. Taking then $F_{i}=T^{-1}\left(F_{i}^{*}\right)$ and $G_{i}=T^{-1}\left(G_{i}^{*}\right)$, we obtain $k$ disjoint capacitors $\left(F_{i}, G_{i}\right)$ on $X$ satisfying $(a)$ with $v=c \frac{\mu(X)}{k}$. In fact, they also satisfy $(b)$ with $\varkappa=C(\gamma+1)$, which follows from the fact that the Dirichlet integral is locally preserved by the mapping $T$, and the degree of $T$ is at most $\gamma+1$. Substituting these values of $v$ and $\varkappa$ into (1.4), we obtain (1.5).

Since the measure $\mu^{*}$ on $\mathbb{S}^{2}$ is a pull-back of a measure on $X$, one may not have enough control over how $\mu^{*}$ is distributed on $\mathbb{S}^{2}$, contrary to the capacity cap*, which is related to the standard metric on $\mathbb{S}^{2}$. Nevertheless, suppose for a moment, that one can find $k$ geodesic balls $B_{i}$ on $\mathbb{S}^{2}$ such that

$$
\mu^{*}\left(B_{i}\right) \geq v
$$

and the balls $2 B_{i}$ are disjoint (here $2 B$ denotes the ball with the same center as $B$ and with the radius equal to twice the radius of $B$ ). It is easy to show that the capacity cap* $(B, 2 B)$ admits an upper bound by an absolute constant $C$ (see inequality (5.7) in the proof of Theorem 5.3). Hence, in this case, both conditions $\left(a^{\prime}\right)$ and $\left(b^{\prime}\right)$ are satisfied for the capacitors $\left(B_{i}, 2 B_{i}\right)$.

However, in general one may not find $k$ disjoint balls on $\mathbb{S}^{2}$ with the property (1.7). Korevaar introduced a very ingenious and extremely elaborate method for choosing more complicated sets to be used for the capacitors in questions. His argument was designed to spot the places of concentration of measure $\mu^{*}$ on $\mathbb{S}^{2}$, while still having control over the corresponding capacities. This method was further developed by two of the authors [27] in the setting of abstract measure metric spaces. ${ }^{3}$

Decomposition of a metric measure space by annuli. In this paper we present a new, significantly simpler method of constructing the capacitors satisfying the above properties $(a)$ and $(b)$. Let $(X, d)$ be a metric space. By an annulus in $X$ we mean any set $A \subset X$ of the following form

$$
A=\{x \in X: r \leq d(x, a)<R\},
$$

\footnotetext{
${ }^{2}$ The constant $8 \pi$ in (1.6) is sharp for the case $\gamma=0$, that is for a sphere, but not in general. The sharp value for a torus was obtained by Nadirashvili [46]. For non-oriented surfaces, the sharp estimate for $\lambda_{2}$ of a projective plane was obtained by $\mathrm{Li}$ and Yau [40], and for a Klein bottle - by Jakobson, Nadirashvili, and Polterovich [34].

${ }^{3}$ For another general method of obtaining upper bounds for higher eigenvalues see [8], [9]. For lower bounds of eigenvalues via capacities see [23], [42, Theorem 2.3.2/1].
} 
where $a \in X$ and $0 \leq r<R<\infty$ (in particular, if $r=0$ then $A$ is the ball $B(x, R))$. Also, denote by $2 A$ the following annulus:

$$
2 A=\left\{x \in X: \frac{1}{2} r \leq d(x, a)<2 R\right\} .
$$

The following theorem is a key result that underpins the above approach for constructing disjoint capacitors and estimating of the eigenvalues.

Theorem 1.1. (=Corollary 3.12) Let $(X, d)$ be a metric space satisfying the following covering property: there exists a constant $N$ such that any metric ball of radius $r$ in $X$ can be covered by at most $N$ balls of radii $r / 2$. Let all metric balls in $X$ be precompact sets, and let $\nu$ be a non-atomic Radon measure on $X$. Then, for any positive integer $k$, there exists a sequence $\left\{A_{i}\right\}_{i=1}^{k}$ of $k$ annuli in $X$ such that, for any $i=1,2, \ldots, k$,

$$
\nu\left(A_{i}\right) \geq c \frac{\nu(X)}{k},
$$

and the annuli $2 A_{i}$ are disjoint. Here $c$ is a positive constant depending only on $N$.

If in addition one has a properly defined capacity cap on $X$ such that $\operatorname{cap}(B, 2 B) \leq$ $Q$ for any ball $B$ in $X$ then one can show that $\operatorname{cap}(A, 2 A) \leq 4 Q$ for any annulus $A$ in $X$. Thus, applying Theorem 1.1 to $X=\mathbb{S}^{2}$ with the measure $\nu=\mu^{*}$ we obtain $k$ capacitors $\left(A_{i}, 2 A_{i}\right)$ satisfying $\left(a^{\prime}\right)$ and $\left(b^{\prime}\right)$, thus giving a new proof of the theorem of Korevaar (see Corollary 4.11 and Theorem 5.4 for more details).

A modification of the above method can be used to estimate the eigenvalues of a Schrödinger type operator. Let $X$ be as above a Riemannian manifold, and fix a function $q \in L_{l o c}^{1}(X, \mu)$. Consider the operator

$$
L=-\Delta-q
$$

and the associated energy form

$$
\mathcal{E}_{q}[f]:=\int_{X} f L f d \mu=\int_{X}\left(|\nabla f|^{2}-q f^{2}\right) d \mu,
$$

defined for all $f \in \mathcal{F}:=C_{0}^{\infty}(X)$. For any real $\lambda$, define the counting function $\mathcal{N}_{\lambda}(L)$ as the supremum of the dimensions of all vector spaces $\mathcal{V} \subset \mathcal{F}$ such that

$$
\mathcal{E}_{q}[f]<\lambda\|f\|_{L^{2}(X, \mu)}^{2} \quad \text { for all } f \in \mathcal{V}, f \not \equiv 0 .
$$

If the operator $L$ with the domain $\mathcal{F}$ admits the Friedrichs extension to a self-adjoint operator in $L^{2}(X, \mu)$ (also denoted by $L$ ), then

$$
\mathcal{N}_{\lambda}(L)=\operatorname{dim} \operatorname{Im} \mathbf{1}_{(-\infty, \lambda)}(L) .
$$

In particular, if the spectrum of $L$ below $\lambda$ is discrete then $\mathcal{N}_{\lambda}(L)$ is just the number of the eigenvalues of $L$, which are smaller than $\lambda$, counted with the multiplicity. In the case $\lambda=0$ we will also use the notation

$$
\operatorname{Neg}(L)=\mathcal{N}_{0}(L) .
$$

Denote by $B(x, r)$ the geodesic ball on $X$ of radius $r$ centered at $x \in X$. The following result is a particular case of Theorem 5.3.

Theorem 1.2. Let $X$ be a complete Riemannian manifold. Assume that, for some constants $N$ and $M$, the following is true:

(i) any ball $B(x, r)$ in $X$ can be covered by at most $N$ balls of radii $r / 2$; 
(ii) for all $x \in X$ and $r>0$,

$$
\mu(B(x, r)) \leq M r^{2} .
$$

Then, for any function $q \in L_{l o c}^{1}(X, \mu)$,

$$
N e g(-\Delta-q) \geq c \int_{X} q_{\delta} d \mu
$$

where

$$
q_{\delta}:=\delta q_{+}-q_{-},
$$

the constant $\delta \in(0,1)$ depends only on $N$, and the constant $c>0$ depends on $N$ and $M$.

The right hand side of (1.10) is undefined if $\int_{X} q_{+} d \mu=\int_{X} q_{-} d \mu=\infty$. In this case, let us set it to be $-\infty$ so that the statement is still trivially valid.

The constant $\delta$ comes from the proof for technical reasons. We conjecture that in fact one can take $\delta=1$ so that $q_{\delta}=q$ and

$$
N e g(-\Delta-q) \geq c \int_{X} q d \mu \text {. }
$$

If $q \geq 0$ then certainly (1.11) follows from (1.10) just by renaming $c \delta$ by $c$. It would be interesting to obtain (1.11) also for a signed function $q$.

Note also that the hypothesis (1.9) is essential for the result: without it the estimate (1.11) may fail even for positive $q$ (see Example 4.20).

Let us mention for comparison a theorem of Cwickel-Lieb-Rozenblum saying that, for any non-negative function $q$ in $\mathbb{R}^{n}, n>2$,

$$
N e g(-\Delta-q) \leq C \int_{\mathbb{R}^{n}} q^{n / 2} d \mu
$$

where $C$ depends only on $n$ (see, for example, $[\mathbf{2 8}],[\mathbf{3 8}],[\mathbf{5 1}]$ ). It is known that (1.12) is not true in $\mathbb{R}^{2}$ whereas, by Theorem 1.2 , the opposite inequality (1.11) holds in $\mathbb{R}^{2}$.

Applying Theorem 1.2 to the potential $q+\lambda$ instead of $q$ and using elementary estimates for $(q+\lambda)_{\delta}$ we obtain that, for any $\lambda \in \mathbb{R}$,

$$
\mathcal{N}_{\lambda}(L) \geq c \int_{X} q_{\delta^{2}} d \mu+c^{\prime} \lambda \mu(X),
$$

where $c^{\prime}=c \delta$. If the spectrum of $L$ is discrete and hence consists of an increasing sequence $\left\{\lambda_{k}(L)\right\}_{k=1}^{\infty}$ of eigenvalues counted with the multiplicities, then (1.13) implies

$$
\lambda_{k}(L) \leq \frac{C k-\int_{X}\left(\delta q_{+}-\delta^{-1} q_{-}\right) d \mu}{\mu(X)},
$$

where $C$ depends on $N$ and $M$. Again, in would be interesting to prove (1.14) with $\delta=1$, that is

$$
\lambda_{k}(L) \leq \frac{C k-\int_{X} q d \mu}{\mu(X)} .
$$

In the case when the operator $L$ is positive definite, we have proved that

$$
\lambda_{k}(L) \leq \frac{C k-\int_{X} q d \mu}{\varepsilon \mu(X)},
$$


where $\varepsilon \in(0,1)$ depends on $N$ (see Theorem 5.15). Note that the function $q$ here may be signed, and the positivity of $L$ implies $\int_{X} q d \mu \leq 0$.

We deduce Theorem 5.3 (and hence Theorem 1.2) from more general Theorems $4.1,4.17$, which estimate the counting function of an abstract energy form on a (pseudo)metric space. The idea of the proof is as follows. Assume for simplicity that $q \geq 0$. In order to estimate from below $N e g(-\Delta-q)$ we will construct $k$ disjoint capacitors $\left(F_{i}, G_{i}\right)$ on $X$ such that $\operatorname{cap}\left(F_{i}, G_{i}\right)$ is controlled from above while $\nu\left(F_{i}\right)$ is controlled from below, where the measure $\nu$ is defined by $d \nu=q d \mu$. Indeed, applying Theorem 1.1 to the space $X$ with this measure $\nu$, we obtain $k$ annuli $A_{i}$ such that

$$
\nu\left(A_{i}\right) \geq c \frac{\nu(X)}{k}
$$

and $2 A_{i}$ are disjoint. The hypothesis (1.9) implies that

$$
\operatorname{cap}\left(A_{i}, 2 A_{i}\right) \leq C M \text {. }
$$

Assuming that $k$ is taken so that

$$
C M<c \frac{\nu(X)}{k},
$$

we choose nearly optimal test functions for the capacitors $\left(A_{i}, 2 A_{i}\right)$ and consider the linear space $\mathcal{V}$ spanned by them. Then $\mathcal{V}$ is a $k$-dimensional subspace of $C_{0}^{\infty}(X)$ such that for any $f \in \mathcal{V} \backslash\{0\}$

$$
\int_{X}|\nabla f|^{2} d \mu<\int_{X} f^{2} d \nu
$$

that is $\mathcal{E}_{q}[f]<0$, whence it follows that $\operatorname{Neg}(-\Delta-q) \geq k$. Taking the largest $k$ satisfying (1.15), we obtain

$$
N e g(-\Delta-q) \geq\left\lfloor\frac{c}{C M} \nu(X)\right\rfloor=\left\lfloor c^{\prime} \int_{X} q d \mu\right\rfloor .
$$

An additional argument allows to get rid of the floor function here and to obtain (1.11).

If as above $X=\left(\Sigma_{\gamma}, g\right)$ then, using the conformal mapping $T$ between $X$ and $\mathbb{S}^{2}$, one obtains from Theorem 1.2 the following extension of the Korevaar estimate (1.5): for any function $q \in L_{l o c}^{1}(X)$ and for all $k \geq 1$,

$$
\lambda_{k}(-\Delta-q) \leq \frac{C(\gamma+1) k-\int_{X}\left(\delta q_{+}-\delta^{-1} q_{-}\right) d \mu}{\mu(X)},
$$

where $C>0$ and $\delta>0$ are absolute constants (see Theorem 5.4).

Stability index of minimal surfaces. Another application of Theorem 1.2 occurs for minimal surfaces ${ }^{4}$ in $\mathbb{R}^{3}$. Let $X$ be a complete oriented immersed minimal surface in $\mathbb{R}^{3}$. The Jacobi operator (or the stability operator) of $X$ is the operator $L=-\Delta-2 K$, where $\Delta$ is the Laplace operator on $X$ associated with the induced Riemannian metric, and $K$ is the Gauss curvature of $X$. By definition, the stability index ind $(X)$ of the minimal surface $X$ is $N e g(L)$. It is well known that if $\operatorname{ind}(X)<\infty$ then the total curvature

$$
K_{\text {total }}(X):=\int_{X}|K| d \mu
$$

\footnotetext{
${ }^{4}$ For a detailed account of minimal surfaces see the surveys $[\mathbf{1 3}]$ and $[\mathbf{4 3}]$ in the same volume.
} 
is finite, and $X$ has a finite number of ends and a finite genus. Theorem 1.2 allows to prove the following result (see also Theorem 6.6).

Theorem 1.3. Let $X$ be a connected complete oriented minimal surface immersed in $\mathbb{R}^{3}$ and let ind $(X)<\infty$. If $X$ has $k$ ends and all the ends are embedded then

$$
\operatorname{ind}(X) \geq \frac{c}{k} K_{\text {total }}(X),
$$

where $c$ is an absolute positive constant.

The factor $\frac{1}{k}$ in (1.17) comes from an estimate of the constant $M$ in (1.9). We conjecture that in fact

$$
\operatorname{ind}(X) \geq c K_{\text {total }}(X)
$$

Note that the inequality in the opposite direction is true for any minimal surface (not necessarily complete) - see [28] and the discussion in Section 6.2 below. As a step towards (1.18) we prove the following result.

Theorem 1.4. (=Corollary 6.8) Let X be a connected complete oriented minimal surface embedded in $\mathbb{R}^{3}$. Then

$$
\operatorname{ind}(X) \geq c \sqrt{K_{\text {total }}(X)}
$$

where $c$ is an absolute positive constant.

The inequality (1.19) is obtained from (1.17) and the following estimate:

$$
\text { ind }(X) \geq k-1 \text {, }
$$

where $k$ is the number of ends of $X$ (it suffices to assume that ind $(X)<\infty$ ). The latter estimate is proved in Theorem 6.7 using the techniques specific to minimal surfaces.

Manifolds of higher dimension. Let $(X, g)$ be a compact Riemannian manifold of dimension $n \geq 2, \mu$ be its Riemannian measure, and $q \geq 0$ be a $L_{l o c}^{1}$-function on $X$. We claim that

$$
N e g(-\Delta-q) \geq \frac{c}{\mu(X)^{n / 2-1}}\left(\int_{X} q d \mu\right)^{n / 2},
$$

where $c$ is a positive constant depending only on the conformal class of the Riemannian metric $g$ (see Theorem 5.9 and Example 5.12).

Assuming for simplicity that $q$ is positive and continuous, we obtain from (1.20) that, for all $k=1,2, \ldots$,

$$
\lambda_{k}\left(\frac{-\Delta}{q}\right) \leq C \frac{\mu(X)}{\int_{X} q d \mu}\left(\frac{k}{\mu(X)}\right)^{2 / n},
$$

where $C=c^{-2 / n}$. In the case $q \equiv 1$ this estimate was proved by Korevaar [37]. 
Eigenvalues of a boundary surface. Let us mention another amusing application of the estimate (1.5). Let $M$ be a 3-dimensional Cartan-Hadamard manifold, and let $\Omega \subset M$ be a bounded open set with a smooth boundary $\Gamma$ that is diffeomorphic to $\Sigma_{\gamma}$. Let $\lambda_{k}(\Omega)$ denote the $k$-th smallest eigenvalue of the Laplace operator in $\Omega$ with the Dirichlet boundary condition, and $\lambda_{k}(\Gamma)$ be the $k$-th smallest eigenvalue of the Laplace operator on $\Gamma$ (in both cases, $k$ starts with 1 ). Then, for any $k=1,2, \ldots$, we have

$$
\lambda_{k}(\Omega) \geq \frac{c}{\gamma+1} \frac{\lambda_{k+1}(\Gamma)}{k^{1 / 3}}
$$

where $c$ is an absolute positive constant (see Theorem 5.14).

Higher order operators. Let $m$ be a positive integer, and consider in $\mathbb{R}^{n}$ the operator

$$
L=(-\Delta)^{m}-q
$$

where $q \in L_{l o c}^{1}$. One defines the counting functions $\mathcal{N}_{\lambda}(L)$ and $N e g(L)$ in the same way as above using the associated energy form. We claim that if $n=2 m$ and $q \geq 0$ then

$$
N e g\left((-\Delta)^{m}-q\right) \geq c \int_{\mathbb{R}^{n}} q d \mu
$$

where $c=c(n)>0$ (see Example 4.19).

Fractals sets. As we have already mentioned, the main estimates of counting functions in Theorems 4.1, 4.17 are obtained in the general setting of energy forms on metric spaces. This makes it possible to apply the present results to fractal sets. Without going into details of the theory of fractals ${ }^{5}$, let us just say that a fractal set can typically be regarded as a metric space $(X, d)$ endowed with a Radon measure $\mu$ and an energy functional $\mathcal{E}$. The properties of these spaces resemble many properties of $\mathbb{R}^{n}$ but with fractional dimensions.

Let $B(x, r)$ denote a ball of the metric $d$. Then, normally, there exists a positive exponent $\alpha$ such that

$$
\mu(B(x, r)) \simeq r^{\alpha}
$$

for all $x \in X$ and $r>0$ (or for a bounded range of $r$ if $X$ is bounded). With the energy functional $\mathcal{E}$ one associates the capacity $\operatorname{cap}_{\mathcal{E}}$ defined similarly to (1.2) (see Section 2.2 for details), which normally admits the following estimate:

$$
\operatorname{cap}_{\mathcal{E}}(B(x, r), B(x, 2 r)) \simeq r^{\alpha-\beta},
$$

where $\beta>0$. The next result (which is a particular case of Corollary 4.14) is obtained using the techniques based on Theorem 1.1.

Theorem 1.5. Let $(X, d)$ be a metric space, $\mu$ be a non-atomic Radon measure on $X$ such that $0<\mu(X)<\infty$, and $\mathcal{E}$ be a local, positive definite, closable energy form on $(X, \mu)$, whose generator $H$ has a discrete spectrum. Assume that, for some positive constants $N, C_{1}, C_{2}$,

(i) any ball $B(x, r)$ in $X$ can be covered by at most $N$ balls of radii $r / 2$;

(ii) for some $\alpha>0$ and $\beta \geq \alpha$ and for any ball $B(x, r)$ in $X$, the following estimates hold:

$$
\mu(B(x, r)) \leq C_{1} r^{\alpha} \quad \text { and } \quad \operatorname{cap}_{\mathcal{E}}(B(x, r), B(x, 2 r)) \leq C_{2} r^{\alpha-\beta} .
$$

\footnotetext{
${ }^{5}$ For a detailed account of fractals, we refer the reader to lecture notes $[\mathbf{2}]$ by Barlow as well as to his article [4] in this volume. See also [25] for function theory on fractal spaces.
} 
Then, for all $k=1,2, \ldots$,

$$
\lambda_{k}(H) \leq C\left(\frac{k}{\mu(X)}\right)^{\beta / a},
$$

where the constant $C$ depends on $N, C_{1}, C_{2}, \alpha, \beta$.

The structure of the paper. In Section 2 we introduce the abstract notion of an energy form and the associated capacity, prove their general properties, and give examples.

In Section 3 we present the proof of Theorem 1.1.

In Section 4 we prove general estimates for the counting function of an abstract energy form, using decomposition of a metric space by capacitors.

In Section 5 we estimate the counting function and the eigenvalues of Schrödinger type operators on Riemannian manifolds and Riemann surfaces (most of these results were surveyed above).

In Section 6 we apply these estimates to the Jacobi operator and deduce lower bounds of the stability index of minimal surfaces in $\mathbb{R}^{3}$.

Acknowledgment. The first author is thankful to David Hoffman for the useful discussions about minimal surfaces.

\section{Energy forms on measure spaces}

2.1. Energy form. Let $X$ be a topological space and let $C_{0}(X)$ be the space of all continuous functions on $X$ with compact supports, endowed with the supnorm.

Definition 2.1. A $C_{0}$-energy form $(\mathcal{E}, \mathcal{F})$ on a topological space $X$ is a symmetric bilinear form $\mathcal{E}(f, g)$, defined on a dense subspace $\mathcal{F} \subset C_{0}(X)$.

Below, we will introduce also $L^{2}$-energy forms. By default, by an energy form we will mean a $C_{0}$-energy form.

An energy form $(\mathcal{E}, \mathcal{F})$ is called positive definite if $\mathcal{E}[f] \geq 0$ for all $f \in \mathcal{F}$. An energy form $(\mathcal{E}, \mathcal{F})$ is called local if $\mathcal{E}(f, g)=0$ whenever function $f, g \in \mathcal{F}$ have disjoint supports. The form $(\mathcal{E}, \mathcal{F})$ is called strongly local if, for all $f, g \in \mathcal{F}$,

$$
f \equiv \text { const in a neighborhood of } \operatorname{supp} g \Longrightarrow \mathcal{E}(f, g)=0 \text {. }
$$

Clearly, a strongly local energy form is local.

A measure $\mu$ on $X$ is called a Radon measure if $\mu$ is defined on all Borel sets of $X$ and $\mu$ is finite on all compact sets. A couple $(X, \mu)$ is called a measure space if $X$ is a topological space and $\mu$ is a Radon measure on $X$. In the presence of measure, we can consider more general energy forms.

Definition 2.2. An $L^{2}$-energy form $(\mathcal{E}, \mathcal{F})$ in a measure space $(X, \mu)$ is a symmetric bilinear form $\mathcal{E}(f, g)$, defined on a dense subspace $\mathcal{F} \subset L^{2}(X, \mu)$.

Clearly, any $C_{0}$-energy form is also an $L^{2}$-energy form. Note also that any signed Radon measure $\sigma$ can be considered as an energy form with domain $C_{0}(X)$, as follows:

$$
\sigma(f, g):=\int_{X} f g d \sigma
$$

Moreover, (2.1) is defined for all $f, g \in L^{2}(X,|\sigma|)$ (where $|\sigma|$ is the total variation of $\sigma$ ) so that $(2.1)$ defines an $L^{2}$-energy form in $L^{2}(X,|\sigma|)$. 
An $L^{2}$-energy form $(\mathcal{E}, \mathcal{F})$ is called semi-bounded below if there exists a constant $K \geq 0$ such that for all $f \in \mathcal{F}$

$$
\mathcal{E}[f]:=\mathcal{E}(f, f) \geq-K\|f\|_{2},
$$

where $\|f\|_{2}$ is the $L^{2}(X, \mu)$-norm of $f$. In particular, a positive definite form is semi-bounded below with the constant $K=0$.

An $L^{2}$-energy form $(\mathcal{E}, \mathcal{F})$ is called closed if it is semi-bounded below and the domain $\mathcal{F}$ is a Hilbert space with respect to the inner product

$$
(f, g)_{\mathcal{E}}:=\mathcal{E}(f, g)+(K+1) \mu(f, g),
$$

where $K$ is the constant from (2.2).

An $L^{2}$-energy form $(\mathcal{E}, \mathcal{F})$ is called closable if it is semi-bounded below and, for any sequence $\left\{f_{n}\right\} \subset \mathcal{F}$,

$$
\left\|f_{n}\right\|_{2} \rightarrow 0 \quad \text { and } \quad \mathcal{E}\left[f_{n}-f_{m}\right] \rightarrow 0 \quad \Longrightarrow \quad \mathcal{E}\left[f_{n}\right] \rightarrow 0 .
$$

It is well-known that a closable $L^{2}$-form $(\mathcal{E}, \mathcal{F})$ has a unique extension to a subspace $\widetilde{\mathcal{F}}$ of $L^{2}(X, \mu)$ so that $(\mathcal{E}, \widetilde{\mathcal{F}})$ is closed and $\mathcal{F}$ is dense in $\widetilde{\mathcal{F}}$ with respect to the inner product $(2.3)$. The extension of $\mathcal{E}$ to $\widetilde{\mathcal{F}}$ is also denoted by $\mathcal{E}$, and the form $(\mathcal{E}, \widetilde{\mathcal{F}})$ is called the closure of $(\mathcal{E}, \mathcal{F})$.

2.2. Capacity. Let $X$ be a topological space. For any Borel set $G \subset X$ denote by $C_{0}(G)$ the set of all continuous functions $f$ on $X$ such that supp $f$ is compact and is contained in the interior of $G$. Any couple $(F, G)$ of Borel subsets of $X$ such that $F \subset G$, will be referred to as a capacitor.

Let $(\mathcal{E}, \mathcal{F})$ be a positive definite energy form on $X$. For any capacitor $(F, G)$, define the class $\mathcal{T}(F, G)$ of test functions as follows:

$$
\mathcal{T}(F, G):=\left\{f \in \mathcal{F} \cap C_{0}(G): 0 \leq f \leq 1, f=1 \text { in a neighborhood of } \bar{F}\right\},
$$

and define the capacity $\operatorname{cap}_{\mathcal{E}}(F, G)$ by

$$
\operatorname{cap}_{\mathcal{E}}(F, G):=\inf _{f \in \mathcal{T}(F, G)} \mathcal{E}[f] .
$$

If $\mathcal{T}(F, G)$ is empty then $\operatorname{cap}_{\mathcal{E}}(F, G)=+\infty$.

For a general theory of capacities see $[\mathbf{2 0}]$ or $[\mathbf{4 2}]$. Here we will need only two elementary facts.

Lemma 2.3. Let $(\mathcal{E}, \mathcal{F})$ be a positive definite energy form. If $F \subset G \subset F^{\prime} \subset G^{\prime}$ are Borel sets then

$$
\operatorname{cap}_{\mathcal{E}}\left(F^{\prime} \backslash G, G^{\prime} \backslash F\right)^{1 / 2} \leq \operatorname{cap}_{\mathcal{E}}(F, G)^{1 / 2}+\operatorname{cap}_{\mathcal{E}}\left(F^{\prime}, G^{\prime}\right)^{1 / 2}
$$

Proof. If $\mathcal{T}(F, G)$ or $\mathcal{T}\left(F^{\prime}, G^{\prime}\right)$ is empty then (2.7) trivially holds. Otherwise, observe that if $f \in \mathcal{T}(F, G)$ and $g \in \mathcal{T}\left(F^{\prime}, G^{\prime}\right)$ then the function $g-f$ is in $\mathcal{T}\left(F^{\prime} \backslash G, G^{\prime} \backslash F\right)$ (see Fig. 1).

Since the form $\mathcal{E}$ is positive definite, it satisfies the Cauchy-Schwarz inequality inequality. Hence, we obtain

$$
\begin{aligned}
\operatorname{cap}_{\mathcal{E}}\left(F^{\prime} \backslash G, G^{\prime} \backslash F\right) & \leq \mathcal{E}[g-f] \\
& =\mathcal{E}[f]+\mathcal{E}[g]-2 \mathcal{E}(f, g) \\
& \leq \mathcal{E}[f]+\mathcal{E}[g]+2 \sqrt{\mathcal{E}[f] \mathcal{E}[g]} \\
& =(\sqrt{\mathcal{E}[f]}+\sqrt{\mathcal{E}[g]})^{2} .
\end{aligned}
$$




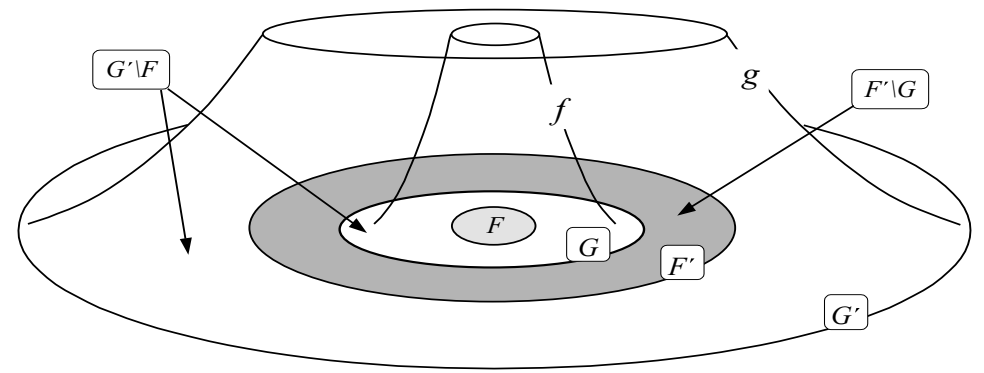

Figure 1. Capacitor $\left(F^{\prime} \backslash G, G^{\prime} \backslash F\right)$

Taking infimum over $f$ and $g$, we obtain (2.6).

Remark 2.4. If the form $(\mathcal{E}, \mathcal{F})$ is in addition strongly local then (2.6) improves as follows:

$$
\operatorname{cap}_{\mathcal{E}}\left(F^{\prime} \backslash G, G^{\prime} \backslash F\right) \leq \operatorname{cap}_{\mathcal{E}}(F, G)+\operatorname{cap}_{\mathcal{E}}\left(F^{\prime}, G^{\prime}\right) .
$$

Indeed, in the above notation, $f \in C_{0}(G)$ and $\left.g\right|_{F^{\prime}} \equiv 1$ imply that $g \equiv 1$ in a neighborhood of $\operatorname{supp} f$, whence $\mathcal{E}(f, g)=0$. Hence, we obtain

$$
\operatorname{cap}_{\mathcal{E}}\left(F^{\prime} \backslash G, G^{\prime} \backslash F\right) \leq \mathcal{E}[g-f]=\mathcal{E}[f]+\mathcal{E}[g],
$$

whence (2.7) follows.

Lemma 2.5. Let $(\mathcal{E}, \mathcal{F})$ be a strongly local positive definite energy form. If $E \subset F \subset G$ are Borel sets then

$$
\operatorname{cap}_{\mathcal{E}}(E, F)^{-1}+\operatorname{cap}_{\mathcal{E}}(F, G)^{-1} \leq \operatorname{cap}_{\mathcal{E}}(E, G)^{-1} .
$$

ProOF. Note that by the monotonicity property of the capacity,

$$
\operatorname{cap}_{\mathcal{E}}(E, F) \geq \operatorname{cap}_{\mathcal{E}}(E, G) \text { and } \operatorname{cap}_{\mathcal{E}}(F, G) \geq \operatorname{cap}_{\mathcal{E}}(E, G) .
$$

Therefore, if one of the capacities $\operatorname{cap}_{\mathcal{E}}(E, F), \operatorname{cap}_{\mathcal{E}}(F, G)$ is equal to $\infty$ then $(2.8)$ follows from (2.9). Otherwise the classes $\mathcal{T}(E, F)$ and $\mathcal{T}(F, G)$ are non-empty. Observe that, for any test functions $f \in \mathcal{T}(E, F)$ and $g \in \mathcal{T}(F, G)$, the function $h=t f+(1-t) g$ belongs to $\mathcal{T}(E, G)$, for any $t \in[0,1]$ (see Fig. 2).

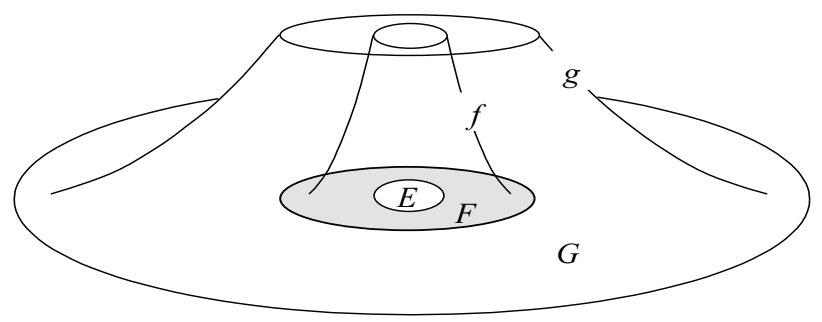

Figure 2. Capacitors $(E, F)$ and $(F, G)$

By the strong locality, $f \in C_{0}(F)$ and $\left.g\right|_{F} \equiv 1$ imply $\mathcal{E}(f, g)=0$, whence

$$
\operatorname{cap}_{\mathcal{E}}(E, G) \leq \mathcal{E}[h]=t^{2} \mathcal{E}[f]+(1-t)^{2} \mathcal{E}[g] .
$$


If $\mathcal{E}[f]=\mathcal{E}[g]=0$ then $(2.10)$ yields $\operatorname{cap}_{\mathcal{E}}(E, G)=0$, and $(2.8)$ is trivially satisfied. Otherwise, taking in $(2.10) t=\frac{\mathcal{E}[g]}{\mathcal{E}[f]+\mathcal{E}[g]}$, we obtain

$$
\operatorname{cap}_{\mathcal{E}}(E, G) \leq \frac{\mathcal{E}[f] \mathcal{E}[g]}{\mathcal{E}[f]+\mathcal{E}[g]}
$$

whence (2.8) follows.

2.3. Generator. Let $(X, \mu)$ be a measure space and $(\mathcal{E}, \mathcal{F})$ be a $L^{2}$-energy form on $X$.

Definition 2.6. A densely defined linear operator $H$ in $L^{2}(X, \mu)$ is called a generator of the form $(\mathcal{E}, \mathcal{F})$ if $\operatorname{dom}(H) \subset \mathcal{F}$ and

$$
\mathcal{E}(f, g)=\mu(H f, g) \quad \text { for all } f \in \operatorname{dom}(H) \text { and } g \in \mathcal{F} .
$$

It follows from (2.11) that a generator is a symmetric operator, in the sense that

$$
\mu(H f, g)=\mu(f, H g) \quad \text { for all } f, g \in \operatorname{dom}(H) .
$$

Conversely, any densely defined symmetric operator $H$ in $L^{2}(X, \mu)$ determines the $L^{2}$-energy form $\left(\mathcal{E}_{H}, \operatorname{dom}(H)\right)$ by

$$
\mathcal{E}_{H}(f, g):=\mu(H f, g) .
$$

Clearly, $H$ is a generator of $\mathcal{E}_{H}$.

If the form $(\mathcal{E}, \mathcal{F})$ is closable then its closure $(\mathcal{E}, \widetilde{\mathcal{F}})$ has a unique generator $H$, which is a self-adjoint operator on $L^{2}(X, \mu)$ (see for example [16, Theorem 4.4.2]). We will refer to $H$ as the self-adjoint generator of $(\mathcal{E}, \widetilde{\mathcal{F}})$, and also of $(\mathcal{E}, \mathcal{F})$ (although $H$ may be not a generator of $(\mathcal{E}, \mathcal{F})$ ). If in addition the operator $H$ is non-negative definite then $H^{1 / 2}$ is defined. In it known that $\operatorname{dom}\left(H^{1 / 2}\right)=\widetilde{\mathcal{F}}$ and

$$
\mathcal{E}(f, g)=\mu\left(H^{1 / 2} f, H^{1 / 2} g\right) \quad \text { for all } f, g \in \widetilde{\mathcal{F}}
$$

(see [15]). If $H$ is a self-adjoint, semi-bounded below operator in $L^{2}(X, \mu)$ then the form $\mathcal{E}_{H}$ defined by (2.12) is closable, and its self-adjoint generator is $H$.

2.4. Counting function. Let $(\mathcal{E}, \mathcal{F})$ be an $L^{2}$-energy form on a measure space $(X, \mu)$. Define the counting function $\mathcal{N}_{\lambda}(\mathcal{E}, \mu)$ as the supremum of the dimensions of all linear spaces $\mathcal{V} \subset \mathcal{F}$ such that

$$
\mathcal{E}[f]<\lambda \mu[f] \text { for any } f \in \mathcal{V} \backslash\{0\},
$$

where $\lambda$ is a real parameter; that is,

$$
\mathcal{N}_{\lambda}(\mathcal{E}, \mu):=\sup \{\operatorname{dim} \mathcal{V}: \mathcal{V} \prec \mathcal{F} \text { and } \mathcal{E}[f]<\lambda \mu[f] \forall f \in \mathcal{V} \backslash\{0\}\},
$$

where the relation $\mathcal{V} \prec \mathcal{F}$ means that $\mathcal{V}$ is a linear subspace of $\mathcal{F}$. If the family of spaces $\mathcal{V}$ in (2.15) is empty then we assign to the sup the value 0 .

If $(\mathcal{E}, \mathcal{F})$ is a $C_{0}$-energy form on $X$ then $\mathcal{N}_{0}(\mathcal{E}, \mu)$ does not depend on $\mu$ so we will use in this case notation $N e g(\mathcal{E})$; that is

$$
N e g(\mathcal{E}):=\sup \{\operatorname{dim} \mathcal{V}: \mathcal{V} \prec \mathcal{F} \text { and } \mathcal{E}[f]<0 \forall f \in \mathcal{V} \backslash\{0\}\} \text {. }
$$

For a closable $L^{2}$-energy form $(\mathcal{E}, \mathcal{F})$, consider also the modified counting function

$$
\mathcal{N}_{\lambda}^{*}(\mathcal{E}, \mu)=\sup \{\operatorname{dim} \mathcal{V}: \mathcal{V} \prec \widetilde{\mathcal{F}} \text { and } \mathcal{E}[f] \leq \lambda \mu[f] \forall f \in \mathcal{V}\},
$$


where $\widetilde{\mathcal{F}}$ is the domain of the closure of $(\mathcal{E}, \mathcal{F})$. Note that there are two differences between $\mathcal{N}_{\lambda}^{*}(\mathcal{E}, \mu)$ and $\mathcal{N}_{\lambda}(\mathcal{E}, \mu)$ : using $\widetilde{\mathcal{F}}$ instead of $\mathcal{F}$ and using a non-strict inequality.

Lemma 2.7. Let $(\mathcal{E}, \mathcal{F})$ be a closable $L^{2}$-energy form on a measure space $(X, \mu)$ and let $H$ be its self-adjoint generator. Then, for any real $\lambda$, we have

$$
\mathcal{N}_{\lambda}(\mathcal{E}, \mu)=\operatorname{dim} \operatorname{Im} \mathbf{1}_{(-\infty, \lambda)}(H)
$$

and

$$
\mathcal{N}_{\lambda}^{*}(\mathcal{E}, \mu)=\operatorname{dim} \operatorname{Im} \mathbf{1}_{(-\infty, \lambda]}(H) .
$$

Here $\mathbf{1}_{A}$ is the indicator function of the set $A \subset \mathbb{R}$, and the operators $\mathbf{1}_{(-\infty, \lambda)}(H)$ are $\mathbf{1}_{(-\infty, \lambda]}(H)$ are understood in the sense of spectral theory. In particular, if the spectrum of $H$ is discrete then $\operatorname{dim} \operatorname{Im} \mathbf{1}_{(-\infty, \lambda)}(H)$ is the number of the eigenvalues of $H$ below $\lambda$, counted with the multiplicities, and $\operatorname{dim} \operatorname{Im} \mathbf{1}_{(-\infty, \lambda]}(H)$ is the number of the eigenvalues of $H$, which are at most $\lambda$, also counted with the multiplicities.

The two quantities $\operatorname{dim} \operatorname{Im} \mathbf{1}_{(-\infty, \lambda)}(H)$ and $\operatorname{dim} \operatorname{Im} \mathbf{1}_{(-\infty, \lambda]}(H)$ are normally referred to as the counting functions of the operator $H$. Here we prefer to give a more general definition of the counting functions as follows. For any densely defined symmetric operator $H$ in $L^{2}(X, \mu)$ let us define the counting function $\mathcal{N}_{\lambda}(H)$ by

$$
\mathcal{N}_{\lambda}(H):=\mathcal{N}_{\lambda}\left(\mathcal{E}_{H}, \mu\right)
$$

where the form $\mathcal{E}_{H}$ is defined by (2.12). Also, set $N e g(H)=\mathcal{N}_{0}(H)$.

If the operator $H$ is self-adjoint and semi-bounded below then the form $\mathcal{E}_{H}$ is closable and its self-adjoint generator is $H$. Therefore, by (2.18) we obtain that

$$
\mathcal{N}_{\lambda}(H)=\operatorname{dim} \operatorname{Im} \mathbf{1}_{(-\infty, \lambda)}(H) .
$$

For such an operator $H$, define also the modified counting function by

$$
\mathcal{N}_{\lambda}^{*}(H):=\mathcal{N}_{\lambda}^{*}\left(\mathcal{E}_{H}, \mu\right) .
$$

Then by (2.19) we obtain that

$$
\mathcal{N}_{\lambda}^{*}(H)=\operatorname{dim} \operatorname{Im} \mathbf{1}_{(-\infty, \lambda]}(H) .
$$

Hence, the identities (2.20) and (2.22) justify the above definitions of $\mathcal{N}_{\lambda}(H)$ and $\mathcal{N}_{\lambda}^{*}(H)$.

Proof of Lemma 2.7. Without loss of generality, we can assume that $\mathcal{E}$ is positive definite, and hence, the generator $H$ is also positive definite. For any $\lambda<0$ all terms in (2.18) and (2.19) vanish, so we can assume in the sequel $\lambda \geq 0$. that is

Let $\left\{E_{t}\right\}_{t \in \mathbb{R}}$ be the spectral resolution of the operator $H$ in $L^{2}=L^{2}(X, \mu)$,

$$
E_{t}=\mathbf{1}_{(-\infty, t)}(H)=\mathbf{1}_{[0, t)}(H) .
$$

By spectral theory, for any $f \in L^{2}(X, \mu)$,

$$
\|f\|^{2}=\int_{[0,+\infty)} d\left\|E_{t} f\right\|^{2}
$$

(where $\|\cdot\|$ is the norm in $L^{2}$ ), and, for any $f \in \widetilde{\mathcal{F}}=\operatorname{dom}\left(H^{1 / 2}\right)$,

$$
\mathcal{E}[f]=\left\|H^{1 / 2} f\right\|^{2}=\int_{[0,+\infty)} t d\left\|E_{t} f\right\|^{2} .
$$


Let us first prove (2.19). To prove the inequality

$$
\mathcal{N}_{\lambda}^{*}(\mathcal{E}, \mu) \leq \operatorname{dim} \operatorname{Im} \mathbf{1}_{[0, \lambda]}(H),
$$

it suffices to show that, for any finitely dimensional subspace $\mathcal{V} \subset \widetilde{\mathcal{F}}$ satisfying (2.17), we have

$$
\operatorname{dim} \mathcal{V} \leq \operatorname{dim} \operatorname{Im} \mathbf{1}_{[0, \lambda]}(H) .
$$

Assuming the contrary, we find a function $v \in \mathcal{V} \backslash\{0\}$ such that $v$ is orthogonal to $\operatorname{Im} \mathbf{1}_{[0, \lambda]}(H)$. In particular, $E_{t} v=0$ for all $t \leq \lambda$ so that from (2.23) and (2.24) imply

$$
\|v\|^{2}=\int_{[\lambda,+\infty)} d\left\|E_{t} v\right\|^{2} \quad \text { and } \quad \mathcal{E}[v]=\int_{[\lambda,+\infty)} t d\left\|E_{t} v\right\|^{2}
$$

whence $\mathcal{E}[v] \geq \lambda\|v\|^{2}$. By $v \in \mathcal{V}$ the opposite inequality also holds, that is $\mathcal{E}[v]=$ $\lambda\|v\|^{2}$. In the view of (2.26) it is only possible when, for any $\varepsilon>0$,

$$
\int_{[\lambda+\varepsilon,+\infty)} d\left\|E_{t} v\right\|^{2}=0
$$

which implies that $v \in \operatorname{Im} \mathbf{1}_{\{\lambda\}}(H)$, while $v$ is orthogonal to this space.

To prove the opposite inequality, that is

$$
\mathcal{N}_{\lambda}^{*}(\mathcal{E}, \mu) \geq \operatorname{dim} \operatorname{Im} \mathbf{1}_{[0, \lambda]}(H),
$$

let us observe that, for any $w \in \operatorname{Im} \mathbf{1}_{[0, \lambda]}(H)$,

$$
\|w\|^{2}=\int_{[0, \lambda]} d\left\|E_{t} v\right\|^{2} \text { and } \mathcal{E}[v]=\int_{[0, \lambda]} t d\left\|E_{t} v\right\|^{2},
$$

whence $\mathcal{E}[w] \leq \lambda\|w\|^{2}$. Therefore, the space $\mathcal{V}:=\operatorname{Im}_{(-\infty, \lambda]}(H) \subset \widetilde{\mathcal{F}}$ satisfies (2.17), which implies (2.27).

Let us now prove (2.18), that is

$$
\mathcal{N}_{\lambda}(\mathcal{E}, \mu)=\operatorname{dim} \operatorname{Im} E_{\lambda} .
$$

The inequality

$$
\mathcal{N}_{\lambda}(\mathcal{E}, \mu) \leq \operatorname{dim} \operatorname{Im} E_{\lambda}
$$

is proved similarly to (2.25). Assume from the contrary that there exists a finitely dimensional subspace $\mathcal{V} \subset \mathcal{F}$ satisfying $(2.14)$ and such that $\operatorname{dim} \mathcal{V}>\operatorname{dim} \operatorname{Im} E_{\lambda}$. Then there exists a vector $v \in \mathcal{V} \backslash\{0\}$ such that $v$ is orthogonal to $\operatorname{Im} E_{\lambda}$. Therefore, $E_{t} v=0$ for all $t \leq \lambda$, which implies (2.26) and hence $\mathcal{E}[v] \geq \lambda\|v\|^{2}$, which contradicts $v \in \mathcal{V}$.

Before we prove the opposite inequality, that is

$$
\operatorname{dim} \operatorname{Im} E_{\lambda} \leq \mathcal{N}_{\lambda}(\mathcal{E}, \mu)
$$

let us verify that

$$
\mathcal{E}[w]<\lambda\|w\|^{2} \text { for any } w \in \operatorname{Im} E_{\lambda} \backslash\{0\} .
$$

Indeed, for all $w \in \operatorname{Im} E_{\lambda}$ we have (2.28), whence $\mathcal{E}[w] \leq \lambda\|w\|^{2}$. In the view of (2.28) the equality is possible only if $w \in \operatorname{Im} \mathbf{1}_{\{\lambda\}}(H)$ which is impossible because the spaces $\operatorname{Im} \mathbf{1}_{\{\lambda\}}(H)$ and $\operatorname{Im} E_{\lambda}$ are orthogonal.

Let us now prove (2.30). If $\operatorname{Im} E_{\lambda} \subset \mathcal{F}$ then taking $\mathcal{V}=\operatorname{Im} E_{\lambda}$ we conclude the proof. However, in general we can only ensure that $\operatorname{Im} E_{\lambda} \subset \widetilde{\mathcal{F}}$. In order to prove 
(2.30) in full generality, we will show that, for any positive integer $n \leq \operatorname{dim} \operatorname{Im} E_{\lambda}$, there exists a subspace $\mathcal{V} \subset \mathcal{F}$ of the dimension $n$ satisfying (2.14).

Consider the following inner product in $\widetilde{\mathcal{F}}$

$$
(f, g)_{\mathcal{E}}:=(f, g)_{L^{2}}+\mathcal{E}(f, g) .
$$

Since $\operatorname{Im} E_{\lambda} \subset \widetilde{\mathcal{F}}$, there exists a sequence $\left\{w_{k}\right\}_{k=1}^{n}$ in $\operatorname{Im} E_{\lambda}$, which is orthonormal with respect to the inner product $(\cdot, \cdot)_{\mathcal{E}}$. Consider the following subspace of $\widetilde{\mathcal{F}}$

$$
\mathcal{W}:=\operatorname{span}\left\{w_{1}, w_{2}, \ldots w_{n}\right\}
$$

and show that there exists $\beta<\lambda$ such that

$$
\mathcal{E}[w] \leq \beta\|w\|^{2} \quad \text { for any } w \in \mathcal{W} .
$$

Indeed, set

$$
S:=\{f \in \mathcal{W}:\|f\|=1\} \quad \text { and } \quad \beta:=\sup _{f \in S} \mathcal{E}[f],
$$

so that (2.32) holds by linearity. Let us verify that $\beta<\lambda$. The sphere $S$ is a compact subset of the finite dimensional space $\mathcal{W}$, and $\mathcal{E}[f]$ is a continuous functional on $\mathcal{W}$. Hence, there is a point $f \in S$ such that $\mathcal{E}[f]=\beta$. On the other hand, by $(2.31)$ we have $\mathcal{E}[f]<\lambda$ for any $f \in S$, whence $\beta<\lambda$.

Since $\mathcal{F}$ is dense in $\widetilde{\mathcal{F}}$ in the norm

$$
\|f\|_{\mathcal{E}}:=(f, f)_{\mathcal{E}}^{1 / 2}=\left(\|f\|^{2}+\mathcal{E}[f]\right)^{1 / 2}
$$

for any $\varepsilon>0$ there exists a sequence $\left\{v_{k}\right\}_{k=1}^{n}$ in $\mathcal{F}$ such that

$$
\left\|v_{k}-w_{k}\right\|_{\mathcal{E}}<\varepsilon \quad \text { for all } k=1,2, \ldots, n \text {. }
$$

Set

$$
\mathcal{V}=\operatorname{span}\left(v_{1}, v_{2}, \ldots, v_{n}\right)
$$

and observe that $\mathcal{V} \subset \mathcal{F}$ and $\operatorname{dim} \mathcal{V}=n$ provided $\varepsilon$ is small enough. Furthermore, let us show that $\varepsilon$ can be chosen so small that

$$
\mathcal{E}[v]<\lambda\|v\|^{2} \quad \text { for any } v \in \mathcal{V} \backslash\{0\},
$$

which would finish the proof of (2.30). For any $v \in \mathcal{V} \backslash\{0\}$ there exists a vector $\xi=\left(\xi_{1}, \xi_{2}, \ldots, \xi_{n}\right) \in \mathbb{R}^{n} \backslash\{0\}$ such that

$$
v=\sum_{k} \xi_{k} v_{k} .
$$

Set

$$
w=\sum_{k} \xi_{k} w_{k}
$$

and observe that $w \in \mathcal{W}$ and

$$
\|w\|_{\mathcal{E}}=\left(\sum_{k} \xi_{k}^{2}\right)^{1 / 2}=|\xi| .
$$


Then we have

$$
\begin{aligned}
\|v-w\|_{\mathcal{E}} & =\left\|\sum_{k} \xi_{k}\left(v_{k}-w_{k}\right)\right\|_{\mathcal{E}} \\
& \leq \sum_{k}\left|\xi_{k}\right|\left\|v_{k}-w_{k}\right\|_{\mathcal{E}} \\
& \leq\left(\sum_{k}\left|\xi_{k}\right|^{2}\right)^{1 / 2}\left(\sum_{k}\left\|v_{k}-w_{k}\right\|_{\mathcal{E}}^{2}\right)^{1 / 2} \\
& \leq|\xi| \sqrt{n} \varepsilon
\end{aligned}
$$

whence

$$
\|v-w\|_{\mathcal{E}} \leq \sqrt{n} \varepsilon\|w\|_{\mathcal{E}} .
$$

In particular, we obtain from $(2.35)$

$$
\|v\| \geq\|w\|-\sqrt{n} \varepsilon\|w\|_{\mathcal{E}}
$$

and

$$
\mathcal{E}[v]^{1 / 2} \leq \mathcal{E}[w]^{1 / 2}+\sqrt{n} \varepsilon\|w\|_{\mathcal{E}}
$$

Setting

and using

$$
R[w]:=\frac{\mathcal{E}[w]^{1 / 2}}{\|w\|} \leq \sqrt{\beta}
$$

$$
\|w\|_{\mathcal{E}} \leq\|w\|+\mathcal{E}[w]^{1 / 2}=(1+R[w])\|w\| \leq(1+\sqrt{\beta})\|w\|=: c\|w\|,
$$

we obtain

$$
\frac{\mathcal{E}[v]^{1 / 2}}{\|v\|} \leq \frac{\mathcal{E}[w]^{1 / 2}+\sqrt{n} \varepsilon c\|w\|}{\|w\|-\sqrt{n} \varepsilon c\|w\|}=\frac{R[w]+\sqrt{n} \varepsilon c}{1-\sqrt{n} \varepsilon c} \leq \frac{\sqrt{\beta}+\sqrt{n} \varepsilon c}{1-\sqrt{n} \varepsilon c} .
$$

Since $\beta<\lambda$, the right hand side here is smaller than $\sqrt{\lambda}$ provided $\varepsilon$ is small enough, whence (2.34) follows.

For the rest of this section we fix an $L^{2}$-energy form $(\mathcal{E}, \mathcal{F})$ on $(X, \mu)$ and use the short notation $\mathcal{N}_{\lambda}=\mathcal{N}_{\lambda}(\mathcal{E}, \mu)$. The function $\lambda \mapsto \mathcal{N}_{\lambda}$ is monotone increasing and takes only values $0,1,2, \ldots,+\infty$. It is useful to observe that this function is left continuous, that is

$$
\mathcal{N}_{\lambda}=\lim _{t \rightarrow \lambda-} \mathcal{N}_{t}
$$

Indeed, let $\mathcal{V}$ be a finite dimensional space satisfying (2.14). As it follows from the compactness argument (see the above proof), there exists $t<\lambda$ such that

$$
\mathcal{E}[v]<t\|v\|^{2} \quad \text { for any } v \in \mathcal{V} \backslash\{0\},
$$

which implies $\mathcal{N}_{t} \geq \operatorname{dim} \mathcal{V}$ and hence (2.38).

It is natural to interpret the jumps of the function $\mathcal{N}_{\lambda}$ as the eigenvalues of the form $\mathcal{E}$. Namely, for any positive integer $k=1,2,3, \ldots$, set

$$
\lambda_{k}=\lambda_{k}(\mathcal{E}, \mu):=\inf \left\{\lambda \in \mathbb{R}: \mathcal{N}_{\lambda} \geq k\right\} .
$$

Here we allow $\lambda_{k}$ to take also values $\pm \infty$. It is straightforward to see that, for any real $\lambda$,

$$
\mathcal{N}_{\lambda}=\sup \left\{k: \lambda_{k}<\lambda\right\}
$$


where the supremum is taken over all positive integers $k$ (see Fig. 3).

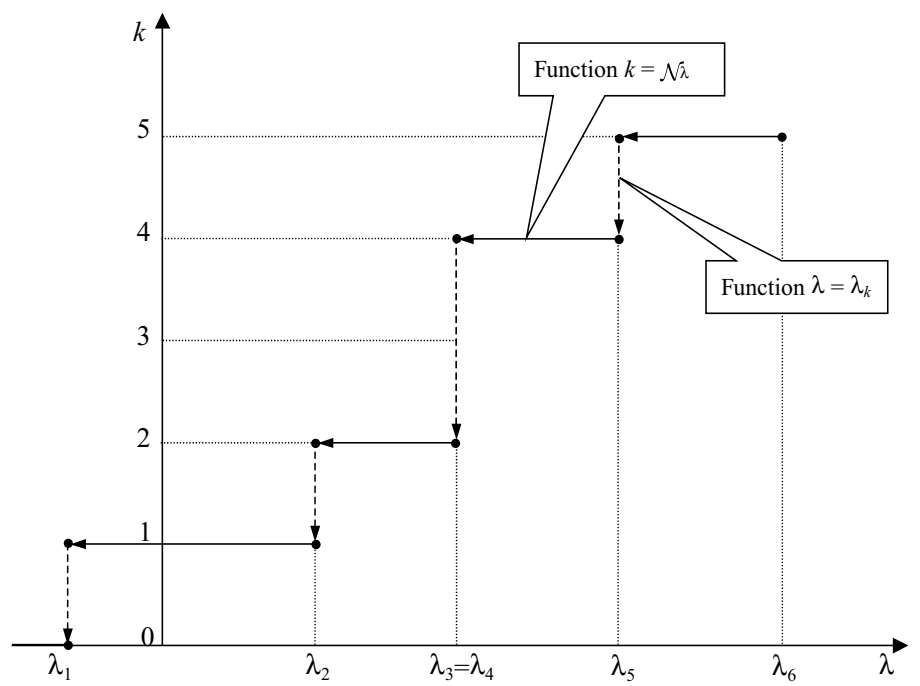

FiguRE 3. Sample graphs of functions $\lambda \mapsto \mathcal{N}_{\lambda}$ (horizontal) and $k \mapsto \lambda_{k}$ (vertical)

The following statement will allow us to switch between the estimates of $\mathcal{N}_{\lambda}$ and those of $\lambda_{k}$.

Lemma 2.8. Let $a, b$ be reals, and $a>0$.

(a) If, for all $\lambda>b$,

$$
\mathcal{N}_{\lambda} \geq\left\lfloor\frac{\lambda-b}{a}\right\rfloor
$$

then, for all $k=1,2, \ldots$

$$
\lambda_{k} \leq a k+b
$$

(b) If, for all $\lambda>b$,

$$
\mathcal{N}_{\lambda} \geq\left\lceil\frac{\lambda-b}{a}\right\rceil
$$

then for all $k=1,2, \ldots$

$$
\lambda_{k} \leq a(k-1)+b .
$$

Here $\lfloor\cdot\rfloor$ is the floor function, that is $\lfloor x\rfloor$ is the maximal integer, which is at most $x$, and $\lceil\cdot\rceil$ is the ceiling function, that is $\lceil x\rceil$ is the minimal integer, which is at least $x$.

Proof. (a) Set $\lambda=a k+b$ and observe that for this $\lambda$, we have $\mathcal{N}_{\lambda} \geq\lfloor k\rfloor=k$. Therefore, by (2.39) we obtain $\lambda_{k} \leq \lambda$, which was to be proved.

(b) Choose $\lambda=a(k-1)+b+\varepsilon$ for some $\varepsilon>0$. Then by the hypothesis

$$
\mathcal{N}_{\lambda} \geq\left\lceil k-1+\frac{\varepsilon}{a}\right\rceil=k,
$$

whence by (2.39) $\lambda_{k} \leq \lambda$. The claim follows by letting $\varepsilon \rightarrow 0$. 
Remark 2.9. Assume that the form $(\mathcal{E}, \mathcal{F})$ is closable and $H$ is its self-adjoint generator. The spectrum $\operatorname{spec}(H)$ is bounded below, and let $\lambda_{\text {ess }}$ be the bottom of the essential spectrum of $H$. In particular, the spectrum of $H$ below $\lambda_{\text {ess }}$ is discrete.

By (2.18), for any $\lambda<\lambda_{\text {ess }}, \mathcal{N}_{\lambda}$ is the sum of the multiplicities of all the eigenvalues of $H$ below $\lambda$, and, for any $\lambda>\lambda_{\text {ess }}, \mathcal{N}_{\lambda}=+\infty$. If $n:=\mathcal{N}_{\lambda_{\text {ess }}}<\infty$ then there are exactly $n$ eigenvalues of $H$ below $\lambda_{\text {ess }}$, and they coincide with $\lambda_{1}, \lambda_{2}, \ldots, \lambda_{n}$, while for all $k>n$ we have $\lambda_{k}=\lambda_{\text {ess }}$. If $n=\infty$ then all the eigenvalues of $H$ below $\lambda_{\text {ess }}$ are given by the sequence $\left\{\lambda_{k}\right\}_{k=1}^{\infty}$.

2.5. Perturbation of an energy form. As already was mentioned above, any signed Radon measure $\sigma$ on a topological space $X$ defines an energy form on $C_{0}(X)$ by

$$
\sigma(f, g):=\int_{X} f g d \sigma .
$$

For any $C_{0}$-energy form $(\mathcal{E}, \mathcal{F})$ on $X$, consider a new energy form $(\mathcal{E}-\sigma, \mathcal{F})$ defined by

$$
(\mathcal{E}-\sigma)(f, g):=\mathcal{E}(f, g)-\sigma(f, g)
$$

which is called the perturbation of the form $(\mathcal{E}, \mathcal{F})$ by the signed measure $\sigma$. If $\sigma$ is a measure then we obviously have the following identity:

$$
\mathcal{N}_{\lambda}(\mathcal{E}, \sigma)=N e g(\mathcal{E}-\lambda \sigma) .
$$

Lemma 2.10. Let an $L^{2}$-energy form $(\mathcal{E}, \mathcal{F})$ be non-negative definite and closable in $L^{2}(X, \mu)$, and let $\sigma$ be a signed Radon measure on $X$, absolutely continuous with respect to $\mu$. If there exist constants $0<c<1$ and $C>0$ such that, for all $f \in \mathcal{F}$,

$$
\sigma_{+}[f] \leq c \mathcal{E}[f]+C \mu[f],
$$

then the form $(\mathcal{E}-\sigma, \mathcal{F})$ is closable.

Proof. Consider first the case when $\sigma_{+}=0$. Then the form $\mathcal{E}-\sigma=\mathcal{E}+\sigma_{-}$ is non-negative definite, and if $\left\{f_{n}\right\}_{n=1}^{\infty}$ is a sequence in $\mathcal{F}$ such that

$$
\mu\left[f_{n}\right] \rightarrow 0 \text { and } \quad\left(\mathcal{E}+\sigma_{-}\right)\left[f_{n}-f_{m}\right] \rightarrow 0 \quad \text { as } n, m \rightarrow \infty,
$$

then also $\mathcal{E}\left[f_{n}-f_{m}\right] \rightarrow 0$. By the closability of the form $(\mathcal{E}, \mathcal{F})$ we conclude that $\mathcal{E}\left[f_{n}\right] \rightarrow 0$. By $(2.43)$ we have also $\sigma_{-}\left[f_{n}-f_{m}\right] \rightarrow 0$ whence it follows that the sequence $\left\{f_{n}\right\}$ converges in $L^{2}\left(X, \sigma_{-}\right)$. Since $\left\{f_{n}\right\}$ converges to 0 in $L^{2}(X, \mu)$ and $\sigma_{-}$is absolutely continuous with respect to $\mu$ then the limit of $\left\{f_{n}\right\}$ in $L^{2}\left(X, \sigma_{-}\right)$ is also 0 . Therefore, $\left(\mathcal{E}+\sigma_{-}\right)\left[f_{n}\right] \rightarrow 0$, which means that the form $\left(\mathcal{E}+\sigma_{-}, \mathcal{F}\right)$ is closable.

In general, note that $\mathcal{E}-\sigma=\left(\mathcal{E}+\sigma_{-}\right)-\sigma_{+}$. By the above argument, the form $\left(\mathcal{E}+\sigma_{-}, \mathcal{F}\right)$ is closable, so rename it to $\mathcal{E}$ and rename $\sigma_{+}$to $\sigma$. Hence, we are left to consider the particular case when $\sigma \geq 0$.

Rewrite (2.42) in the form

$$
(1-c) \mathcal{E}[f] \leq C \mu[f]+(\mathcal{E}-\sigma)[f] .
$$

In particular, it implies that $\mathcal{E}-\sigma$ is semi-bounded below. Let $\left\{f_{n}\right\}_{n=1}^{\infty}$ be a sequence in $\mathcal{F}$ such that

$$
\mu\left[f_{n}\right] \rightarrow 0 \quad \text { and } \quad(\mathcal{E}-\sigma)\left[f_{n}-f_{m}\right] \rightarrow 0 \quad \text { as } n, m \rightarrow \infty .
$$


Then (2.44) and (2.45) imply that $\mathcal{E}\left[f_{n}-f_{m}\right] \rightarrow 0$, and the closability of $(\mathcal{E}, \mathcal{F})$ yields that $\mathcal{E}\left[f_{n}\right] \rightarrow 0$. Using again $(2.45)$ we obtain $\sigma\left[f_{n}-f_{m}\right] \rightarrow 0$, and as in part $(a)$ we conclude that $(\mathcal{E}-\sigma)\left[f_{n}\right] \rightarrow 0$, which was to be proved.

Consider two topological spaces $X$ and $X^{\prime}$. A continuous mapping $T: X \rightarrow X^{\prime}$ is said to be proper if, for any compact set $K \subset X^{\prime}$, the preimage $T^{-1}(K)$ is also compact in $X$. If $T$ is a proper mapping then any signed Radon measure $\sigma$ on $X$ can be lifted to a signed Radon measure $\sigma^{\prime}$ on $X^{\prime}$ by

$$
\sigma^{\prime}(\cdot)=\sigma\left(T^{-1}(\cdot)\right) \text {. }
$$

Obviously, we have then

$$
f \in C_{0}\left(X^{\prime}\right) \Longrightarrow f \circ T \in C_{0}(X) \text { and } \sigma[f \circ T]=\sigma^{\prime}[f] .
$$

Definition 2.11. Let $(\mathcal{E}, \mathcal{F})$ and $\left(\mathcal{E}^{\prime}, \mathcal{F}^{\prime}\right)$ be positive definite $C_{0}$-energy forms on topological spaces $X$ and $X^{\prime}$, respectively. We say that a proper mapping $T: X \rightarrow X^{\prime}$ has the energy degree at most $D$, where $0<D<\infty$, if the form $\mathcal{E}$ is dominated by $\mathcal{E}^{\prime}$ in the following sense:

$$
f \in \mathcal{F}^{\prime} \quad \Longrightarrow \quad f \circ T \in \mathcal{F} \text { and } \mathcal{E}[f \circ T] \leq D \mathcal{E}^{\prime}[f] .
$$

Lemma 2.12. If a proper mapping $T: X \rightarrow X^{\prime}$ has the energy degree at most $D$ then

$$
\operatorname{Neg}(\mathcal{E}-\sigma) \geq N e g\left(D \mathcal{E}^{\prime}-\sigma^{\prime}\right) .
$$

Proof. Let $\mathcal{V}^{\prime}$ be a linear subspace of $\mathcal{F}^{\prime}$ such that

$$
D \mathcal{E}^{\prime}[f]-\sigma^{\prime}[f]<0 \text { for any } f \in \mathcal{V}^{\prime} \backslash\{0\} .
$$

Consider the space

$$
\mathcal{V}=\left\{f \circ T: f \in \mathcal{V}^{\prime}\right\}
$$

By (2.46), $\mathcal{V}$ is a linear subspace of $\mathcal{F}$ and, for any $\varphi=f \circ T \in \mathcal{V} \backslash\{0\}$, we have

$$
\mathcal{E}[\varphi]-\sigma[\varphi] \leq D \mathcal{E}^{\prime}[f]-\sigma^{\prime}[f]<0 .
$$

Finally, (2.47) follows from $\operatorname{dim} \mathcal{V} \geq \operatorname{dim} \mathcal{V}^{\prime}$.

2.6. Weighted Riemannian manifolds. Let $X$ be a Riemannian manifold and $\mu_{0}$ be the Riemannian measure on $X$. For any Radon measure $\mu$ on $X$ (which may be equal to $\mu_{0}$ or not), the couple $(X, \mu)$ is called a weighted manifold. On any weighted manifold $(X, \mu)$, there is a natural energy form $\mathcal{E}_{\mu}$ defined on $C_{0}^{1}(X)$ by

$$
\mathcal{E}_{\mu}(f, g)=\int_{X} \nabla f \cdot \nabla g d \mu
$$

where $\nabla$ is the Riemannian gradient. Clearly, $\mathcal{E}_{\mu}$ is a strongly local positive definite energy form on $X$.

Frequently it is more convenient to define the domain of $\mathcal{E}_{\mu}$ by $\mathcal{F}=\operatorname{Lip}_{0}(X)$ where $\operatorname{Lip}_{0}(X)$ is the space all locally Lipschitz functions on $X$ with compact support (note that for any locally Lipschitz function $f$ on $X$, the gradient $\nabla f$ exists $\mu_{0}$-almost everywhere). This will be our default choice of the domain of $\mathcal{E}_{\mu}$.

Let $\mu$ be absolutely continuous with respect to $\mu_{0}$, and the density $\psi=d \mu / d \mu_{0}$ be a smooth positive function. Then the form $\left(\mathcal{E}_{\mu}, \mathcal{F}\right)$ is closable in $L^{2}(X, \mu)$, and its generator is $-\Delta_{\mu}$, where $\Delta_{\mu}$ is the Laplace operator of $(X, \mu)$, defined by

$$
\Delta_{\mu} f=\psi^{-1} \operatorname{div}(\psi \nabla f),
$$


where div is the Riemannian divergence. If $\mu=\mu_{0}$ is the Riemannian measure then $\mathcal{E}_{\mu}$ is the Riemannian energy form and $\Delta_{\mu}=\Delta=\operatorname{div} \circ \nabla$ is the Laplace-Beltrami operator of $X$.

For any positive integer $m$, consider also the energy form of the order $m$ :

$$
\mathcal{E}_{\mu}^{(m)}(f, g)=\int_{X} \nabla^{m} f \cdot \nabla^{m} g d \mu,
$$

where $m$ is a positive integer and

$$
\nabla^{m}= \begin{cases}\Delta_{\mu}^{\frac{m}{2}}, & m \text { even, } \\ \nabla \Delta_{\mu}^{\frac{m-1}{2}}, & m \text { odd }\end{cases}
$$

(in the case of even $m$, the dot "." in (2.49) denotes the product of scalars, whereas for odd $m$ it is the inner product of vectors). The form $\mathcal{E}_{\mu}^{(m)}$ with the domain $C_{0}^{\infty}(X)$ is closable, and its generator is $\left(-\Delta_{\mu}\right)^{m}$.

Given a signed Radon measure $\sigma$ on a weighted manifold $(X, \mu)$, consider a perturbed form $\mathcal{E}_{\mu}-\sigma$ with the domain $\mathcal{F}$. Its generator is a Schrödinger type operator $-\Delta_{\mu}-\sigma$. In Section 5.2 we will need the following fact.

Lemma 2.13. Let $(X, \mu)$ be a weighted manifold, $\mathcal{E}=\mathcal{E}_{\mu}$ be the energy form of $(X, \mu)$, and $\sigma$ be a signed Radon measure on $X$. Let $K$ be a compact subset of $X$ with empty interior, such that $|\sigma|(K)=0$ and $\operatorname{cap}_{\mathcal{E}}(K, U)=0$ for any open set $U \subset X$ containing $K$. Set $X^{\prime}=X \backslash K, \mu^{\prime}=\left.\mu\right|_{X^{\prime}}, \sigma^{\prime}=\left.\sigma\right|_{X^{\prime}}$, and let $\mathcal{E}^{\prime}$ be the energy form of $\left(X^{\prime}, \mu^{\prime}\right)$. Then

$$
\operatorname{Neg}\left(\mathcal{E}^{\prime}-\sigma^{\prime}\right)=\operatorname{Neg}(\mathcal{E}-\sigma) .
$$

If in addition $\mu(K)=0$ then, for all real $\lambda$,

$$
\mathcal{N}_{\lambda}\left(\mathcal{E}^{\prime}-\sigma^{\prime}, \mu^{\prime}\right)=\mathcal{N}_{\lambda}(\mathcal{E}-\sigma, \mu) .
$$

Proof. We have $\mathcal{F}=\operatorname{Lip}_{0}(X)$ and $\mathcal{F}^{\prime}=\operatorname{Lip}_{0}\left(X^{\prime}\right)$. From $\mathcal{F}^{\prime} \subset \mathcal{F}$ it follows

$$
\mathcal{N}_{\lambda}\left(\mathcal{E}^{\prime}-\sigma^{\prime}, \mu\right) \leq \mathcal{N}_{\lambda}(\mathcal{E}-\sigma, \mu),
$$

so that we need to prove the opposite inequality. To that end, it suffices to show that, for any finite dimensional subspace $\mathcal{V}$ of $\mathcal{F}$ such that

$$
\mathcal{E}[f]<\sigma[f]+\lambda \mu[f] \text { for all } f \in \mathcal{V} \backslash\{0\},
$$

there exists a subspace $\mathcal{V}^{\prime}$ of $\mathcal{F}^{\prime}$ of the same dimension as $\mathcal{V}$ and such that

$$
\mathcal{E}[f]<\sigma[f]+\lambda \mu[f] \quad \text { for all } f \in \mathcal{V}^{\prime} \backslash\{0\} .
$$

Set $\nu:=\sigma+\lambda \mu$ and observe that by (2.52) the bilinear form $\nu(f, g)$ is an inner product in $\mathcal{V}$. We will regard $\mathcal{V}$ as a finite dimensional Euclidean space with this inner product. It follows from $(2.52)$ by a compactness argument that there exists $c<1$ such that

$$
\mathcal{E}[f] \leq c \nu[f] \quad \text { for any } f \in \mathcal{V} .
$$

Let $U$ be a precompact open neighborhood of $K$ in $X$ to be specified below. Since $\sup _{U}|f|$ is a semi-norm in $\mathcal{V}$ and any semi-norm in a finite dimensional space is dominated by any norm, there exists a constant $C=C_{U}$ such that

$$
\sup _{x \in U}|f(x)| \leq C \sqrt{\nu[f]} \text { for any } f \in \mathcal{V} .
$$


Set $\psi=1-\varphi$ where $\varphi \in \mathcal{T}(K, U)$, and define $\mathcal{V}^{\prime}$ by

$$
\mathcal{V}^{\prime}=\{\psi f: f \in \mathcal{V}\} \text {. }
$$

Clearly, $\mathcal{V}^{\prime} \subset \mathcal{F}^{\prime}$ and $\operatorname{dim} \mathcal{V}^{\prime} \leq \operatorname{dim} \mathcal{V}$. Let us show that if $U$ is small enough then $\operatorname{dim} \mathcal{V}^{\prime}=\operatorname{dim} \mathcal{V}$. Indeed, let $\left\{U_{n}\right\}_{n=1}^{\infty}$ be a shrinking sequence of precompact open neighborhoods of $K$ such that the intersection of all $U_{n}$ is $K$. The expression

$$
S_{n}(f):=\sup _{X \backslash U_{n}}|f|
$$

defines an increasing sequence $\left\{S_{n}\right\}$ of semi-norms in $\mathcal{V}$. Since $K$ has no interior, we obtain

$$
\lim _{n \rightarrow \infty} S_{n}(f)=\sup _{X \backslash K}|f|=\sup _{X}|f|,
$$

that is $\lim _{n \rightarrow \infty} S_{n}$ is a norm in $\mathcal{V}$. By a compactness argument, we conclude that $S_{n}$ is a norm in $\mathcal{V}$ for some finite $n$. So, choose $U=U_{n}$ for this $n$. Since $\psi=1$ on $X \backslash U$ we obtain, for any $f \in \mathcal{V}$,

$$
\sup _{X}|\psi f| \geq \sup _{X \backslash U}|f|=S_{n}(f),
$$

which implies that $\psi f \equiv 0$ if and only if $f \equiv 0$, and hence $\operatorname{dim} \mathcal{V}^{\prime}=\operatorname{dim} \mathcal{V}$.

We are left to prove (2.53), which is equivalent to

$$
\mathcal{E}[\psi f]<\nu[\psi f] \text { for any } f \in \mathcal{V} \backslash\{0\} .
$$

Indeed, using $\nabla \psi=-\nabla \varphi,(2.55)$, and (2.54), we obtain

$$
\begin{aligned}
\mathcal{E}[\psi f] & =\int_{X}|\nabla(\psi f)|^{2} d \mu \\
& =\int_{X}|\nabla f|^{2} \psi^{2} d \mu+\int_{U}\left(-2 f \psi \nabla f \cdot \nabla \varphi+f^{2}|\nabla \varphi|^{2}\right) d \mu \\
& \leq \mathcal{E}[f]+2 C \sqrt{\nu[f] \mathcal{E}[f] \mathcal{E}[\varphi]}+C^{2} \nu[f] \mathcal{E}[\varphi] \\
& =(\sqrt{\mathcal{E}[f]}+C \sqrt{\nu[f] \mathcal{E}[\varphi]})^{2} \\
& \leq(\sqrt{c}+C \sqrt{\mathcal{E}[\varphi]})^{2} \nu[f]
\end{aligned}
$$

and

$$
\begin{aligned}
\nu[\psi f] & =\int_{X} f^{2} \psi^{2} d \nu \\
& =\int_{X} f^{2} d \nu+\int_{U} f^{2}\left(\psi^{2}-1\right) d \nu \\
& \geq \nu[f]-\sup _{U}|f|^{2}|\nu|(U) \\
& \geq\left(1-C^{2}|\nu|(U)\right) \nu[f]
\end{aligned}
$$

whence

$$
\frac{\mathcal{E}[\psi f]}{\nu[\psi f]} \leq \frac{(\sqrt{c}+C \sqrt{\mathcal{E}[\varphi]})^{2}}{1-C^{2}|\nu|(U)} .
$$

Note that the best constant $C=C_{U}$ in (2.55) is decreasing when $U$ is shrinking. Since $|\nu|(U) \leq|\sigma|(U)+\lambda \mu(U),|\sigma|(K)=0$, and $\mu(K)=0$ (the latter is needed only in the case $\lambda \neq 0$ ), by choosing $U$ small enough we can make $C_{U}^{2}|\nu|(U)$ 
arbitrarily close to 0 . Since $\operatorname{cap}_{\mathcal{E}}(K, U)=0$ we can choose $\varphi$ to make $\mathcal{E}[\varphi]$ also arbitrarily close to 0 . Finally, using $c<1$, we see that the right hand side of (2.57) can be made smaller than 1 , whence (2.56) follows.

2.7. Fractal spaces. By fractal spaces we mean the fractal sets ${ }^{6}$ in $\mathbb{R}^{n}$, obtained by certain self-similar constructions, like the celebrated Sierpinski gasket. Recall that the Sierpinski gasket $S G$ is constructed from a unit equilateral triangle $T$ in $\mathbb{R}^{2}$ by, firstly, removing the triangle with the vertices in the middles of the sides of $T$, then removing similar middle triangles from the three remaining equilateral triangles with the sides $\frac{1}{2}$, and so on (see Fig. 4).

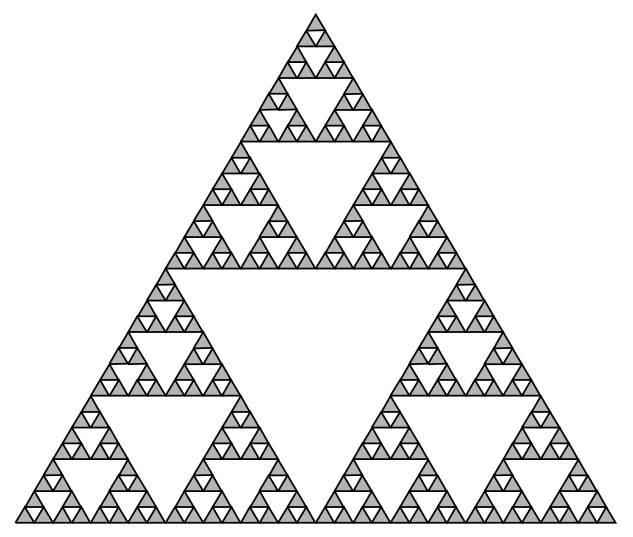

Figure 4. Construction of the Sierpinski gasket: after 5 steps.

One can define a distance function $d$ on $S G$ as the induced Euclidean distance from $\mathbb{R}^{2}$, and a measure $\mu$ on $S G$ as the Hausdorff measure $\mathcal{H}_{\alpha}$ where $\alpha$ is the Hausdorff dimension of $S G$ (in fact, $\alpha=\log _{2} 3$ ). Furthermore, approximating $S G$ by a sequence of graphs and considering a scaling limit of discrete energy forms on the approximating graphs, one defines a positive definite local energy form $(\mathcal{E}, \mathcal{F})$ on $S G$, which is closable in $L^{2}(S G, \mu)$. Moreover, its closure $(\mathcal{E}, \widetilde{\mathcal{F}})$ is a regular Dirichlet form (see for example [2]).

Similar structures can be introduced on most other fractals sets. Let $(X, d)$ be a metric space, $\mu$ be a Radon measure on $X$, and $(\mathcal{E}, \mathcal{F})$ be a positive definite energy form on $X$. If $X$ is obtained by a self-similar construction like above then normally the metric, measure, and the energy structures on $X$ exhibit certain homogeneity. Denote by $B(x, r)$ the metric ball of the radius $r$ centered $x \in X$. Typically the following estimates hold on fractal spaces, for some positive parameters $\alpha$ and $\beta$ :

$$
\mu(B(x, r)) \simeq r^{\alpha}
$$

and

$$
\operatorname{cap}_{\mathcal{E}}(B(x, r), B(x, 2 r)) \simeq r^{\alpha-\beta} .
$$

Here the sign $\simeq$ means that the ratio of the left hand side and the right hand side is bounded from above and below by positive constants, for the specified range of the arguments. The relations (2.58) and (2.59) are supposed to be true for all $x \in X$ and for some range $0<r<R$ of the radius.

\footnotetext{
${ }^{6}$ For detailed account of fractals we refer the reader to $[\mathbf{2}],[\mathbf{3}],[\mathbf{4}],[\mathbf{3 6}]$.
} 
The parameter $\alpha$ from (2.58) is equal to the Hausdorff dimension of $(X, d)$. The nature of the parameter $\beta$, which is called the walk dimension, is more complicated (see [26] or [25]). For example, for $S G$ we have $\beta=\log _{2} 5$. The Euclidean space $\mathbb{R}^{n}$ with the Lebesgue measure $\mu$ satisfies (2.58) with $\alpha=n$, and the standard energy form $\mathcal{E}_{\mu}$ in $\mathbb{R}^{n}$ satisfies $(2.59)$ with $\beta=2$. Moreover, the capacity of the form $\mathcal{E}_{\mu}^{(m)}$ in $\mathbb{R}^{n}$ (defined by $(2.49)$ ) satisfies

$$
\operatorname{cap}_{\mathcal{E}_{\mu}^{(m)}}(B(x, r), B(x, 2 r))=c_{n, m} r^{n-2 m},
$$

where $c_{n, m}>0$, that is $(2.59)$ with $\beta=2 m$ (see $[\mathbf{4 2}]$ ). Hence, in this case $\beta$ is equal to the order of the generator $(-\Delta)^{m}$.

Also in the general case $\beta$ can be regarded as the order of the generator of the energy form. For most fractal spaces one has $\beta>2$ although the generator is always a Markov operator (that is, satisfies the maximum principle) unlike the operators of order $>2$ in $\mathbb{R}^{n}$.

We will come back to fractal spaces in Section 4.3.

\section{Decomposition of a pseudometric space by annuli}

In this section we prove Theorem 3.5, which is the main technical tool of this paper.

Definition 3.1. Given a set $X$, we say that a function $d: X \times X \rightarrow[0,+\infty)$ is a pseudometric (or a pseudodistance function) if $d$ is symmetric, that is $d(x, y)=$ $d(y, x)$, and if $d$ satisfies the triangle inequality, that is

$$
d(x, y) \leq d(x, z)+d(z, y)
$$

for all $x, y, z \in X$.

So, unlike the notion of a metric, we allow $d(x, y)=0$ for distinct $x, y$. For any $x \in X$ and $r \geq 0$, define a ball $B(x, r)$ associated with a pseudometric $d$ as follows:

$$
B(x, r)=\{y \in X: d(x, y)<r\} .
$$

Definition 3.2. A couple $(X, d)$ is called a pseudometric space if $X$ is a topological space, $d$ is a pseudometric on $X$, and the function $y \mapsto d(x, y)$ is continuous for any $x \in X$ (consequently, all balls in a pseudometric space are open sets).

In particular, any metric space is a pseudometric space. Given any set $X$ and a pseudometric $d$ on it, one can define a topology on $X$ using the balls of $d$ as a base (although this topology is not necessarily Hausdorff). With this topology, $(X, d)$ is a pseudometric space. However, in applications the set $X$ may be a priori endowed with a different topology, for example, if $X$ is a manifold, in which case we require that this topology is richer that the one induced by $d$.

Typically, a pseudometric space arises as follows. Let $\left(X^{\prime}, d^{\prime}\right)$ be a metric space and let $T: X \rightarrow X^{\prime}$ be a continuous mapping from a topological space $X$ to $X^{\prime}$. Then the identity

$$
d(x, y)=d^{\prime}(T(x), T(y))
$$

defines a continuous pseudometric on $X$ so that $(X, d)$ is a pseudometric space.

Definition 3.3. Given $\kappa>1$ and a positive integer $N$, we say that a pseudometric space $(X, d)$ satisfies $(\kappa, N)$-covering property if, for any ball $B(x, r)$ in $X$, there exists a family of at most $N$ balls of radii $r / \kappa$, which cover $B(x, r)$. 
Lemma 3.4. If a pseudometric space $(X, d)$ satisfies $(\kappa, N)$-covering property then it satisfies $(\lambda, M)$-covering property for any $\lambda>1$ and some $M=M(\lambda, \kappa, N)$.

Proof. Indeed, let $n$ be a positive integer such that $\kappa^{n-1}<\lambda \leq \kappa^{n}$. Since the $(\lambda, M)$-covering property is monotone in $\lambda$, it suffices to assume that $\lambda=\kappa^{n}$. If $n=1$ then the the claim is trivial. Let us make the inductive step from $n$ to $n+1$. Indeed, by the inductive hypothesis, any ball $B(x, r)$ can be covered by at most $M_{n}$ balls $B\left(x_{i}, r / \kappa^{n}\right)$. By the assumption, each ball $B\left(x_{i}, r / \kappa^{n}\right)$ can be covered by at most $N$ balls of radius $r / \kappa^{n+1}$ each. Hence, $B(x, r)$ can be covered by at most $M_{n+1}:=N M_{n}$ balls of radius $r / \kappa^{n+1}$ each, which settles the claim.

It is useful to note that if $(X, d)$ admits a doubling measure $\mu$, that is, a Borel measure $\mu$ such that, for all $x \in X$ and $r>0$ and for some constant $C$,

$$
0<\mu(B(x, 2 r)) \leq C \mu(B(x, r))<\infty,
$$

then $(X, d)$ satisfies $(2, N)$ covering property with $N \leq C^{3}$.

In a pseudometric space $(X, d)$, for any $x \in X$ and $0 \leq r \leq R$ define the annulus

$$
A(x, r, R):=B(x, R)-B(x, r)=\{y \in X: r \leq d(x, y)<R\} .
$$

Note that $A(x, 0, R)=B(x, R)$.

For any annulus $A=A(x, r, R)$ and $\lambda \geq 1$ denote by $\lambda A$ the following annulus:

$$
\lambda A=A\left(x, \lambda^{-1} r, \lambda R\right) .
$$

Similarly, for $B=B(x, r)$ and $\lambda>0$, set $\lambda B=B(x, \lambda r)$.

Theorem 3.5. Let a pseudometric space $(X, d)$ satisfy $(2, N)$-covering property. Let $\nu$ be a Borel measure on $X$, and assume that there exist positive reals $v$ and $\rho$ such that

$$
\forall x \in X \quad \nu(B(x, \rho / 2)) \leq v \quad \text { and } \quad \exists x_{0} \in X \quad \nu\left(B\left(x_{0}, \rho\right)\right)>v .
$$

Then, for any $\lambda>1$, there exists a family $\mathcal{A}$ of $\left\lceil c \frac{\nu(X)}{v}\right\rceil$ annuli in $X$ satisfying the following properties:

(a) $\nu(A) \geq v$ for any $A \in \mathcal{A}$;

(b) the annuli $\{\lambda A\}_{A \in \mathcal{A}}$ are disjoint.

Here $c$ is a positive constant depending only on $\lambda$ and $N$ (for example, one can define it by $\left.c^{-1}=2+4 M\left(200 \lambda^{3}, 2, N\right)\right)$.

Remark 3.6. If $\nu(X)=\infty$ then we interpret $\left\lceil c \frac{\nu(X)}{v}\right\rceil$ as $\infty$. In this case we claim the existence of an infinite (countable) family $\mathcal{A}$ of annuli with the properties (a) and $(b)$.

If $\nu(X)<\infty$ then one cannot have more than $\frac{\nu(X)}{v}$ disjoint sets each with measure at least $v$. Hence, the number $\left\lceil c \frac{\nu(X)}{v}\right\rceil$ of disjoint annuli guarantied by Theorem 3.5 is optimal up to a constant factor.

The hypothesis (3.1) excludes, in particular, a situation when measure $\nu$ is concentrated in a few atoms, in which case the conclusion of Theorem 3.5 is no longer true. Lemma 3.10 will provide a simple sufficient condition for (3.1).

Remark 3.7. For some applications it is useful to know that each annulus in the family $\mathcal{A}$, which is constructed in the proof, either has the internal radius at least $\rho / 2$ or is a ball of the radius at least $\rho / 2$.

We precede the proof of Theorem 3.5 by an elementary lemma. 
Lemma 3.8. Let $B(x, r)$ and $B(y, s)$ be two balls in $(X, d)$ and $\lambda>1$ be a real such that

$$
\lambda B(x, r) \cap \frac{1}{2} B(y, s) \neq \emptyset \quad \text { and } \quad B(x, r) \backslash B(y, s) \neq \emptyset .
$$

Then the following inclusions take place:

$$
\begin{aligned}
B(x, r) & \subset B(y,(2 \lambda+2) r), \\
B(y, s) & \subset B(x,(4 \lambda+3) r), \\
B(y, \eta r) & \subset B(x,(\eta+2 \lambda+1) r) \quad \text { for any } \eta>0 .
\end{aligned}
$$

Proof. The hypotheses (3.2) imply that

$$
\begin{aligned}
d(x, y) & \leq \lambda r+\frac{s}{2} \\
s & \leq d(x, y)+r .
\end{aligned}
$$

(see Fig. 5).

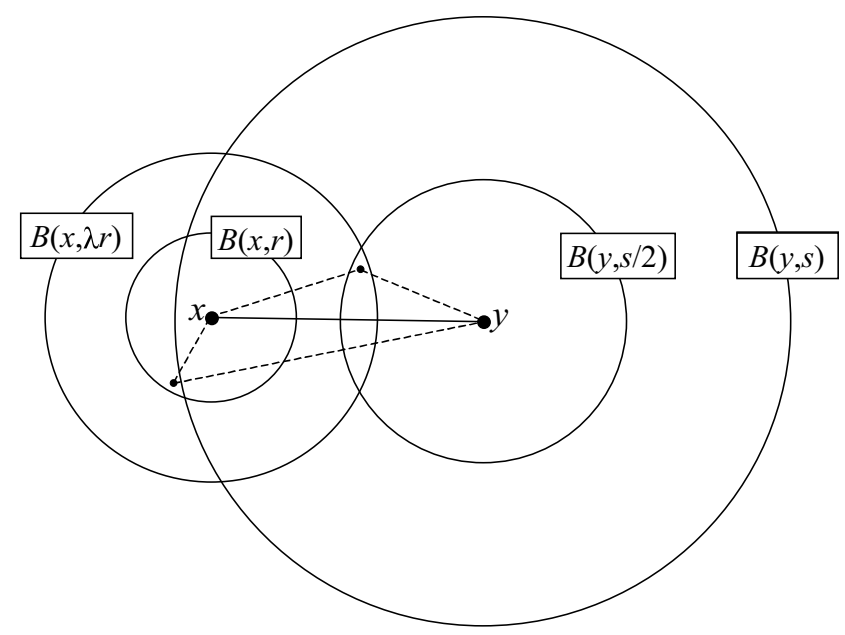

FiguRE 5. Illustration to Lemma 3.8

It follows that

$$
d(x, y) \leq \lambda r+\frac{d(x, y)+r}{2}
$$

and

$$
d(x, y) \leq(2 \lambda+1) r
$$

whence

$$
s \leq(2 \lambda+1) r+r=(2 \lambda+2) r .
$$

Using the above two inequalities, we obtain

$$
\begin{gathered}
B(x, r) \subset B(y, r+d(x, y)) \subset B(y,(2 \lambda+2) r) . \\
B(y, s) \subset B(x, s+d(x, y)) \subset B(x,(4 \lambda+3) r), \\
B(y, \eta r) \subset B(x, \eta r+d(x, y)) \subset B(x,(\eta+2 \lambda+1) r),
\end{gathered}
$$

which was to be proved. 
Proof of Theorem 3.5. Set for simplicity

$$
m(\kappa)=M(\kappa, 2, N),
$$

where $M(\kappa, 2, N)$ is the constant from Lemma 3.4. Hence, any ball of radius $r$ can be covered by at most $m(\kappa)$ balls of radii $r / \kappa$.

Let $n$ be a positive integer such that

$$
n \leq\left\lceil c \frac{\nu(X)}{v}\right\rceil .
$$

We will construct two sequences $\left\{\mathcal{A}_{i}\right\}$ and $\left\{\mathcal{B}_{i}\right\}$ where $i=1,2, \ldots, 2 n, \mathcal{A}_{i}$ is a family of annuli in $X$, and $\mathcal{B}_{i}$ is a family of balls in $X$. These families will satisfy the following properties, for all $i=1,2, \ldots, 2 n$ :

(i) for any $a \in \mathcal{A}_{i}$,

$$
\nu(a) \geq v
$$

(ii) the annuli $\{\lambda a\}_{a \in \mathcal{A}_{i}}$ are disjoint;

(iii) the following inclusion takes place:

$$
\bigcup_{a \in \mathcal{A}_{i}} \lambda a \subset \bigcup_{b \in \mathcal{B}_{i}} \frac{1}{2 \lambda} b ;
$$

(iv) the following inequality takes place:

$$
\nu\left(\bigcup_{b \in \mathcal{B}_{i}} b\right) \leq C v i
$$

where $C=m\left(200 \lambda^{3}\right)$;

(v) $\left|\mathcal{A}_{1}\right|=\left|\mathcal{B}_{1}\right|=1$, and if $i>1$ then

$$
\begin{aligned}
& \text { - either }\left|\mathcal{A}_{i}\right|=\left|\mathcal{A}_{i-1}\right|+1 \text { and }\left|\mathcal{B}_{i}\right| \leq\left|\mathcal{B}_{i-1}\right|+1, \\
& \text { - or }\left|\mathcal{A}_{i}\right|=\left|\mathcal{A}_{i-1}\right| \text { and }\left|\mathcal{B}_{i}\right| \leq\left|\mathcal{B}_{i-1}\right|-1 ;
\end{aligned}
$$

(vi) if $i>1$ then $\mathcal{A}_{i} \supset \mathcal{A}_{i-1}$;

(vii) each annulus in $\mathcal{A}_{i}$ either has the internal radius at least $\rho / 2$ or is a ball of the radius at least $\rho / 2$, and each ball in $\mathcal{B}_{i}$ has the radius at least $\rho$.

Before we actually construct these sequences, let us show how the existence of them proves the theorem. Indeed, each family $\mathcal{A}_{i}$ obviously satisfies $(a)$ and $(b)$. Let us show that one of the families $\mathcal{A}_{i}$ contains at least $n$ annuli. If $n=1$ then we have already $\left|\mathcal{A}_{1}\right|=1$. For an arbitrary $n$, let us verify that $\left|\mathcal{A}_{2 n}\right| \geq n$. To that end, observe that by $(v)$ the numerical sequence $\left\{2\left|\mathcal{A}_{i}\right|-\left|\mathcal{B}_{i}\right|\right\}_{i=1}^{2 n}$ is strictly increasing whereas $2\left|\mathcal{A}_{1}\right|-\left|\mathcal{B}_{1}\right|=1$. This implies

$$
2\left|\mathcal{A}_{2 n}\right|-\left|\mathcal{B}_{2 n}\right| \geq 2 n
$$

and hence $\left|\mathcal{A}_{2 n}\right| \geq n$. If $\nu(X)<\infty$ then this finishes the proof because we can take $n=\left\lceil c \frac{\nu(X)}{v}\right\rceil$. If $\nu(X)=\infty$ then we can do the above construction for any $n$. The union $\mathcal{A}=\bigcup_{i=1}^{\infty} \mathcal{A}_{i}$ is infinite and, since the sequence $\left\{\mathcal{A}_{i}\right\}$ is increasing, $\mathcal{A}$ satisfies the conditions $(a)$ and $(b)$, which finishes the proof in this case. The condition (vii) is not needed for the proof of Theorem 3.5 but settles the claim of Remark 3.7.

Now we construct the families $\mathcal{A}_{i}$ and $\mathcal{B}_{i}$ by induction in $i$. For the inductive basis $i=1$, fix $x_{0} \in X$ and $\rho>0$ satisfying (3.1). Define the set $\mathcal{A}_{1}$ to consist of a single annulus $A\left(x_{0}, 0, \rho\right)$, and the set $\mathcal{B}_{1}$ to consist of a single ball $B\left(x_{0}, 2 \lambda^{2} \rho\right)$. Then the properties $(i),(i i),(i i i),(v),(v i),(v i i)$ are trivially satisfied. To prove 
(iv), let us observe that $B\left(x_{0}, 2 \lambda^{2} \rho\right)$ is covered by at most $m\left(4 \lambda^{2}\right) \leq C$ balls of radii $\rho / 2$, and by (3.1) the measure of each ball of radius $\rho / 2$ is at most $v$. Hence,

$$
\nu\left(B\left(x_{0}, 2 \lambda^{2} \rho\right)\right) \leq C v
$$

which is exactly (3.9). If $n=1$ then the construction of $\mathcal{A}_{1}$ finishes the proof. Hence, in the sequel we assume $n>1$.

Assuming that $i \leq 2 n-1$ and that $\mathcal{A}_{i}$ and $\mathcal{B}_{i}$ are already defined, let us construct $\mathcal{A}_{i+1}$ and $\mathcal{B}_{i+1}$. Set

$$
\bar{r}:=\sup \left\{r: \nu\left(B(x, r) \backslash \bigcup_{b \in \mathcal{B}_{i}} b\right) \leq v \quad \text { for all } x \in X\right\}
$$

and observe that $\bar{r} \geq \rho / 2$ and hence $\bar{r}>0$. It follows from (3.7) and $n>1$ that

$$
n<c \frac{\nu(X)}{v}+1<2 c \frac{\nu(X)}{v} .
$$

Hence, we obtain from (3.9), (3.11), and $i<2 n$ that

$$
\nu\left(X \backslash \bigcup_{b \in \mathcal{B}_{i}} b\right)>\nu(X)-2 n C v>\left(\frac{1}{2 c}-2 C\right) n v>v
$$

because $c^{-1}=2+4 C$ by the definitions of $c$ and $C$. We conclude that there exists a ball $B(x, r)$ such that

$$
\nu\left(B(x, r) \backslash \bigcup_{b \in \mathcal{B}_{i}} b\right)>v
$$

and, furthermore, we can assume that $\bar{r} \leq r<2 \bar{r}$ (see Fig. 6). In particular, we have $r \geq \rho / 2$.

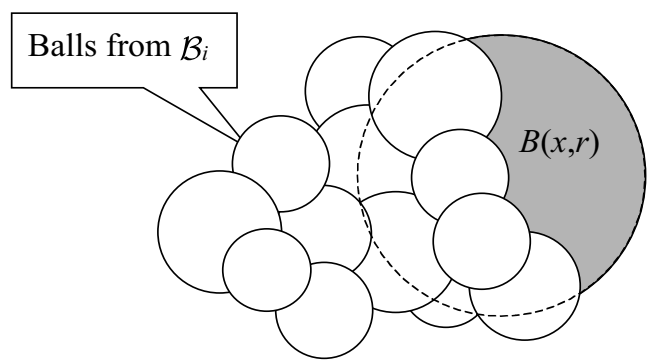

Figure 6 . Choosing ball $B(x, r)$ : the measure of the shaded region is larger than $\alpha$.

Set

$$
k:=\operatorname{card}\left\{b \in \mathcal{B}_{i}: B\left(x, \lambda^{\prime} r\right) \cap \frac{1}{2} b \neq \emptyset\right\}
$$

where

$$
\lambda^{\prime}:=7 \lambda^{2},
$$

and consider the following three cases. 
Case $k=0$. Since $1<\lambda<\lambda^{\prime}$, in this case we have

$$
B(x, \lambda r) \cap \frac{1}{2 \lambda} b=\emptyset \quad \text { for any } b \in \mathcal{B}_{i} .
$$

Define $\mathcal{A}_{i+1}$ and $\mathcal{B}_{i+1}$ by

$$
\mathcal{A}_{i+1}=\mathcal{A}_{i} \cup\{A(x, 0, r)\} \quad \text { and } \quad \mathcal{B}_{i+1}=\mathcal{B}_{i} \cup\left\{B\left(x, 2 \lambda^{2} r\right)\right\} .
$$

Then condition $(i)$ holds by (3.12), and conditions $(i i i),(v),(v i)$ are trivially satisfied. Condition (vii) is satisfied because $r \geq \rho / 2$.

Let us prove $(i i)$. By the inductive hypothesis, we have

$$
\bigcup_{a \in \mathcal{A}_{i}} \lambda a \subset \bigcup_{b \in \mathcal{B}_{i}} \frac{1}{2 \lambda} b
$$

(see Fig. 7)

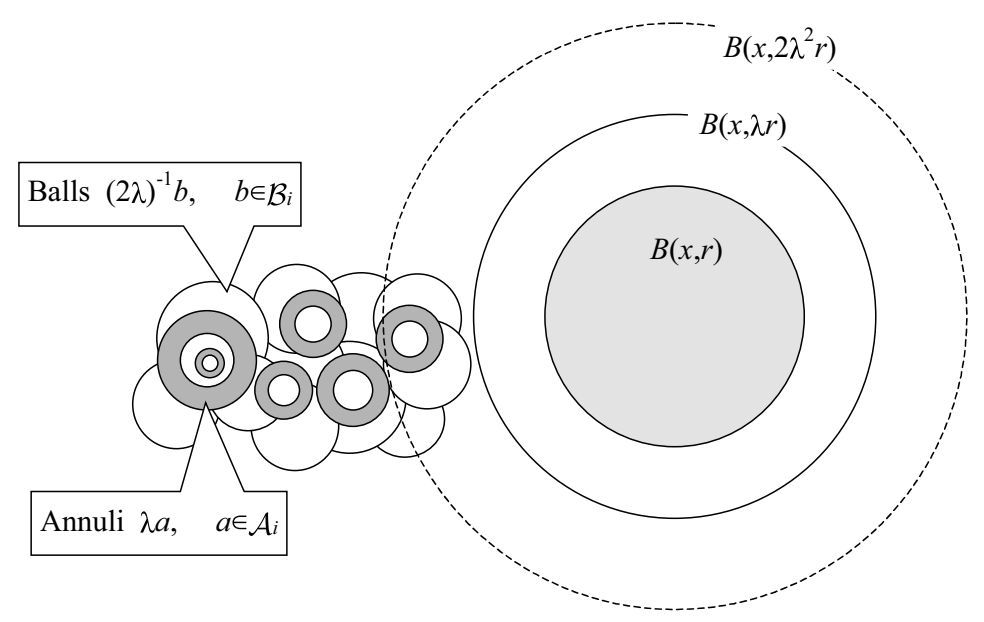

Figure 7. Ball $B(x, \lambda r)$ does not intersect $\frac{1}{2 \lambda} b$, whereas the union of all $\lambda a$ is covered by the union of all $\frac{1}{2 \lambda} b$.

Together with (3.14), this implies that $\lambda A(x, 0, r)=B(x, \lambda r)$ does not intersect any annulus $\lambda a, a \in \mathcal{A}_{i}$, whence (ii) follows.

Let us prove $(i v)$. Since $r / 2<\bar{r}$, it follows from (3.10) that, for any $z \in X$,

$$
\nu\left(B(z, r / 2) \backslash \bigcup_{b \in \mathcal{B}_{i}} b\right) \leq v .
$$

The ball $B\left(x, 2 \lambda^{2} r\right)$ can be covered by at most $m\left(4 \lambda^{2}\right) \leq C$ balls of radii $r / 2$ whence it follows that

$$
\nu\left(B\left(x, 2 \lambda^{2} r\right) \backslash \bigcup_{b \in \mathcal{B}_{i}} b\right) \leq C v .
$$

By the inductive hypothesis, we have

$$
\nu\left(\bigcup_{b \in \mathcal{B}_{i}} b\right) \leq C v i
$$


whence it follows that

$$
\nu\left(\bigcup_{b \in \mathcal{B}_{i+1}} b\right) \leq C v(i+1) .
$$

Case $k \geq 2$. Let us say that a ball $b \in \mathcal{B}_{i}$ is selected if

$$
B\left(x, \lambda^{\prime} r\right) \cap \frac{1}{2} b \neq \emptyset
$$

so that the number of selected balls is exactly $k$. In this case, let us set

$$
\mathcal{A}_{i+1}=\mathcal{A}_{i}
$$

and

where

$$
\mathcal{B}_{i+1}=\mathcal{B}_{i} \backslash\{\text { all selected balls }\} \cup\left\{B\left(x, \lambda^{\prime \prime} r\right)\right\}
$$

$$
\lambda^{\prime \prime}:=14 \lambda \lambda^{\prime}=98 \lambda^{3} \text {. }
$$

Conditions $(i),(i i),(v i),(v i i)$ are trivially satisfied. Condition $(v)$ is satisfied because the number $k$ of the selected balls removed from $\mathcal{B}_{i}$ is at least 2 , whereas only a single ball $B\left(x, \lambda^{\prime \prime} r\right)$ is added.

Let us prove $(i i i)$. Let $b \in \mathcal{B}_{i}$ be a selected ball. By definition, $B\left(x, \lambda^{\prime} r\right) \cap \frac{1}{2} b$ is non-empty and, by (3.12), $B(x, r) \backslash b$ is non-empty, too. Then Lemma 3.8 (inclusion (3.4)) yields

$$
b \subset B\left(x,\left(4 \lambda^{\prime}+3\right) r\right) \subset B\left(x, 7 \lambda^{\prime} r\right)=\frac{1}{2 \lambda} B\left(x, \lambda^{\prime \prime} r\right)
$$

(see Fig. 8).

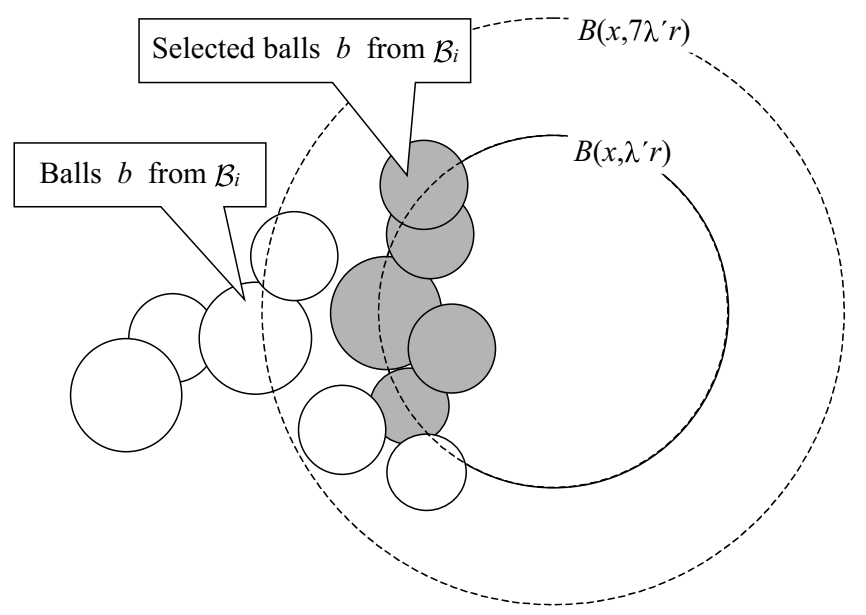

Figure 8. The selected balls (shaded) are inside $B\left(x, 7 \lambda^{\prime} r\right)$.

In particular, the union of all balls $\frac{1}{2 \lambda} b$, where $b$ is selected, is covered by a single ball $\frac{1}{2 \lambda} B\left(x, \lambda^{\prime \prime} r\right)$, whence we obtain

$$
\bigcup_{b \in \mathcal{B}_{i}} \frac{1}{2 \lambda} b \subset \bigcup_{b \in \mathcal{B}_{i+1}} \frac{1}{2 \lambda} b
$$


The claim of $(\mathrm{iii})$ follows then by the inductive hypothesis.

Let us prove $(i v)$. The ball $B\left(x, \lambda^{\prime \prime} r\right)$ can be covered by at most $m\left(2 \lambda^{\prime \prime}\right) \leq C$ balls of radii $r / 2$ whence it follows from (3.15) that

$$
\nu\left(B\left(x, \lambda^{\prime \prime} r\right) \backslash \bigcup_{b \in \mathcal{B}_{i}} b\right) \leq C v .
$$

By definition of $\mathcal{B}_{i+1}$ and by the inductive hypothesis, we obtain

$$
\bigcup_{b \in \mathcal{B}_{i+1}} b \subset\left(\bigcup_{b \in \mathcal{B}_{i}} b\right) \cup\left(B\left(x, \lambda^{\prime \prime} r\right) \backslash \bigcup_{b \in \mathcal{B}_{i}} b\right)
$$

and

$$
\nu\left(\bigcup_{b \in \mathcal{B}_{i+1}} b\right) \leq C v i+C v=C v(i+1) .
$$

Case $k=1$. Set

$$
k_{0}:=\operatorname{card}\left\{b \in \mathcal{B}_{i}: B(x, \lambda r) \cap \frac{1}{2} b \neq \emptyset\right\}
$$

and observe that $k_{0} \leq k$. Hence, either $k_{0}=0$ or $k_{0}=1$. If $k_{0}=0$ then the condition (3.14) is satisfied, and we can argue exactly as in the case $k=0$.

Let us consider the main case $k_{0}=1$. Let $B(y, s) \in \mathcal{B}_{i}$ be the unique ball such that

$$
B(x, \lambda r) \cap \frac{1}{2} B(y, s) \neq \emptyset .
$$

Set

$$
\begin{aligned}
\widetilde{a} & =A\left(y, \frac{1}{2} s, 4 \lambda r\right) \\
\widetilde{b} & =B\left(x, 2 \lambda \lambda^{\prime} r\right)
\end{aligned}
$$

(see Fig. 9) and define the families $\mathcal{A}_{i+1}, \mathcal{B}_{i+1}$ by

$$
\mathcal{A}_{i+1}=\mathcal{A}_{i} \cap\{\widetilde{a}\} \quad \text { and } \quad \mathcal{B}_{i+1}=\mathcal{B}_{i} \cup\{\widetilde{b}\} .
$$

By (3.12) the difference $B(x, r) \backslash B(y, s)$ is non-empty, which together with (3.17) shows that the hypotheses of Lemma 3.8 are satisfied. By inequality (3.6) obtained in the proof of this lemma, we see that $s / 2<4 \lambda r$ so that the annulus $\widetilde{a}$ is welldefined.

Conditions $(v),(v i)$ are obviously true. Condition (vii) is satisfied because by the inductive hypothesis the ball $B(y, s)$ (being an element of $\mathcal{B}_{i}$ ) has the radius $s \geq \rho$ and hence the annulus $\widetilde{a}$ has the internal radius at least $\rho / 2$.

Let us verify $(i)-(i v)$. By Lemma 3.8 we have

$$
B(x, r) \subset B(y,(2 \lambda+2) r) \subset B(y, 4 \lambda r) .
$$

Therefore,

$$
\widetilde{a}=B(y, 4 \lambda r) \backslash B\left(y, \frac{1}{2} s\right) \supset B(x, r) \backslash B(y, s),
$$

which together with (3.12) implies

$$
\nu(\widetilde{a}) \geq \nu\left(B(x, r) \backslash \bigcup_{b \in \mathcal{B}_{i}} b\right) \geq v,
$$




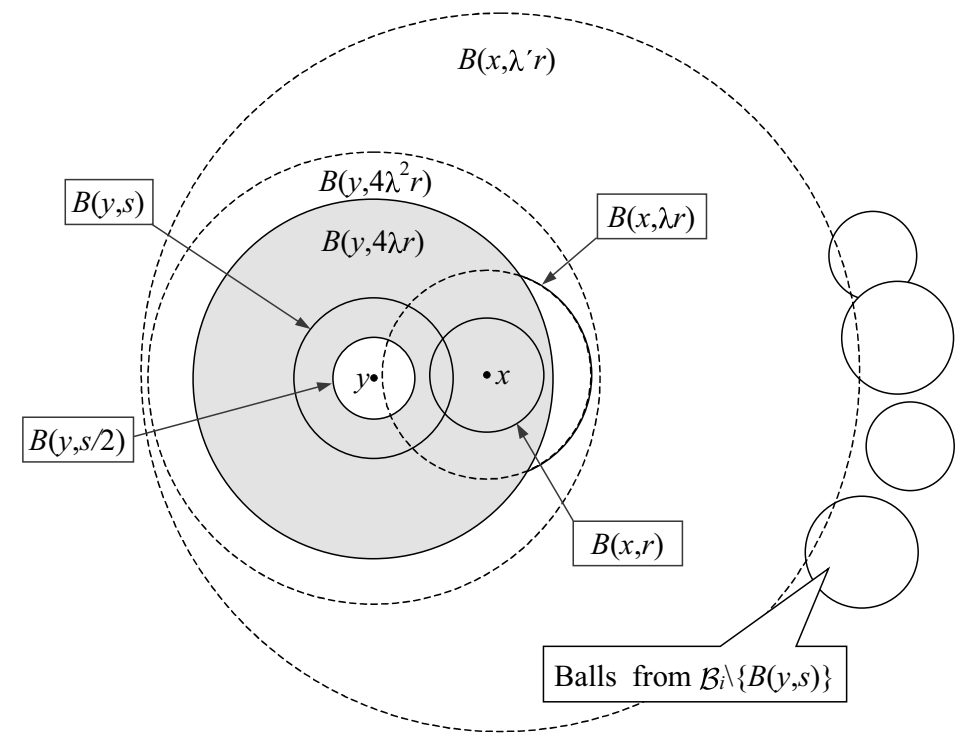

Figure 9. The annulus $\widetilde{a}=A\left(y, \frac{1}{2} s, 4 \lambda r\right)$ is shaded.

whence $(i)$ follows.

To verify $(i i)$ we need to prove that

$$
\lambda \widetilde{a} \cap \lambda a=\emptyset \quad \text { for all } a \in \mathcal{A}_{i} .
$$

It it suffices to prove that

$$
\lambda \widetilde{a} \cap \bigcup_{b \in \mathcal{B}_{i}} \frac{1}{2 \lambda} b=\emptyset,
$$

because by the inductive hypothesis,

$$
\lambda a \subset \bigcup_{b \in \mathcal{B}_{i}} \frac{1}{2 \lambda} b \quad \text { for all } a \in \mathcal{A}_{i} .
$$

To prove (3.18), observe that

$$
\lambda \widetilde{a}=A\left(y, \frac{1}{2 \lambda} s, 4 \lambda^{2} r\right)=B\left(y, 4 \lambda^{2} r\right) \backslash \frac{1}{2 \lambda} B(y, s) .
$$

Applying again Lemma 3.8 to the balls $B(x, r)$ and $B(y, s)$, we obtain by $(3.5)$

$$
B\left(y, 4 \lambda^{2} r\right) \subset B\left(x,\left(4 \lambda^{2}+2 \lambda+1\right) r\right) \subset B\left(x, \lambda^{\prime} r\right),
$$

whence

$$
\lambda \widetilde{a} \subset B\left(x, \lambda^{\prime} r\right) \backslash \frac{1}{2 \lambda} B(y, s) .
$$

In particular, we immediately see that

$$
\lambda \widetilde{a} \cap \frac{1}{2 \lambda} B(y, s)=\emptyset .
$$


By the hypothesis $k=1$, there is a unique ball $b \in \mathcal{B}_{i}$ such that $\frac{1}{2} b$ intersects $B\left(x, \lambda^{\prime} r\right)$, and this ball must be $B(y, s)$. Hence,

$$
B\left(x, \lambda^{\prime} r\right) \cap \frac{1}{2} b=\emptyset \quad \text { for any } b \in \mathcal{B}_{i} \backslash\{B(y, s)\},
$$

whence it follows that

$$
\lambda \widetilde{a} \cap \frac{1}{2 \lambda} b=\emptyset \quad \text { for any } b \in \mathcal{B}_{i} \backslash\{B(y, s)\} .
$$

Clearly, (3.18) follows from (3.20) and (3.21).

Let us verify $($ iii). Indeed, by (3.19) we have

$$
\lambda \widetilde{a} \subset B\left(x, \lambda^{\prime} r\right)=\frac{1}{2 \lambda} \widetilde{b},
$$

which together with the inductive hypothesis settles the claim.

Finally, let us verify $(i v)$. To that end, it suffices to show that

$$
\nu\left(\widetilde{b} \backslash \bigcup_{b \in \mathcal{B}_{i}} b\right) \leq C v .
$$

The ball $\widetilde{b}=B\left(x, 2 \lambda \lambda^{\prime} r\right)$ can be covered by at most $m\left(4 \lambda \lambda^{\prime}\right) \leq C$ balls of radii $r / 2$, whence the claim follows from (3.15).

Definition 3.9. A measure $\nu$ on a pseudometric space $(X, d)$ is called $d$-nonatomic if, for any $x \in X$,

$$
\lim _{r \rightarrow 0} \nu(B(x, r))=0 .
$$

In other words, this means that

$$
\nu(B(x, 0+))=0
$$

where

$$
B(x, 0+):=\bigcap_{r>0} B(x, r)=\{y \in X: d(x, y)=0\} .
$$

If $d$ is a metric then $B(x, 0+)=\{x\}$ and, hence, $\nu$ is $d$-non-atomic if and only if $\nu$ is non-atomic in the usual sense, that is $\nu(\{x\})=0$ for any point $x$.

The following lemma provides a sufficient condition for hypothesis (3.1).

Lemma 3.10. Let $(X, d)$ be a pseudometric space and $\nu$ be a Borel measure on $X$. Assume that

(i) all balls in $X$ are precompact;

(ii) measure $\nu$ is d-non-atomic and $0<\nu(X)<\infty$.

Then, for any $0<v<\nu(X)$, there exists $\rho>0$ satisfying (3.1), that is

$$
\forall x \in X \quad \nu(B(x, \rho / 2)) \leq v \quad \text { and } \exists x_{0} \in X \quad \nu\left(B\left(x_{0}, \rho\right)\right)>v .
$$

Proof. Define on the interval $(0, \infty)$ a function

$$
V(r):=\sup _{x \in X} \nu(B(x, r)) .
$$

It is obvious that $V(r) \rightarrow \nu(X)$ as $r \rightarrow \infty$. Let us verify that $V(r) \rightarrow 0$ as $r \rightarrow 0$. Assuming the contrary, we obtain that there exists a number $\varepsilon>0$ and sequences $\left\{x_{k}\right\}_{k=1}^{\infty} \subset X$ and $\left\{r_{k}\right\}_{k=1}^{\infty} \rightarrow 0$ such that $\nu\left(B\left(x_{k}, r_{k}\right)\right) \geq \varepsilon$ for all $k$. If there is a convergent subsequence $\left\{x_{k_{i}}\right\} \rightarrow x$ then this implies $\nu(B(x, r)) \geq \varepsilon$ for any $r>0$, which contradicts the hypothesis that $\nu$ is $d$-non-atomic. If there 
is no convergent subsequence of $\left\{x_{k}\right\}$ then the compactness of balls implies that the sequence $\left\{x_{k}\right\}$ eventually leaves every ball. Therefore, for any fixed $r>0$, there exists a subsequence $\left\{x_{k_{i}}\right\}$ such that the balls $B\left(x_{k_{i}}, r\right)$ are disjoint. By the finiteness of $\nu(X)$ we obtain that $\nu\left(B\left(x_{k_{i}}, r\right)\right) \rightarrow 0$, whence it follows that $\nu\left(B\left(x_{k_{i}}, r_{k_{i}}\right)\right) \rightarrow 0$.

Fix $r>0$ and set $r_{k}=r / 2^{k}, k=0,1,2, \ldots$ Then $V\left(r_{k}\right) \rightarrow 0$ as $k \rightarrow \infty$ and hence the union of the intervals $\left[V\left(r_{k+1}\right), V\left(r_{k}\right)\right)$ is $(0, V(r))$. Therefore, for any $v \in(0, V(r))$, there exists an index $k$ such that

$$
V\left(r_{k+1}\right) \leq v<V\left(r_{k}\right) \text {. }
$$

Letting $r \rightarrow \infty$, we obtain that for any $v \in(0, \nu(X))$ there exists $\rho>0$ such that

$$
V(\rho / 2) \leq v<V(\rho)
$$

whence (3.22) follows.

Let us mention for the record that the radius $\rho$ from (3.22) satisfies

$$
\rho \geq V^{-1}(v),
$$

where $V^{-1}$ is the generalized inverse to $V(r)$.

Corollary 3.11. Let $(X, d)$ be a pseudometric space and $\nu$ be a Radon measure on $X$. Assume that

(i) $X$ satisfies $(2, N)$-covering property:

(ii) all balls in $X$ are precompact;

(iii) measure $\nu$ is d-non-atomic.

Then, for any $0<v<\nu(X)$ and any positive integer $n \leq\left\lceil c \frac{\nu(X)}{v}\right\rceil$ there exists a family $\mathcal{A}$ of $n$ annuli in $X$ satisfying the following properties:

(a) $\nu(A) \geq v$ for any $A \in \mathcal{A}$;

(b) the annuli $\{2 A\}_{A \in \mathcal{A}}$ are disjoint.

Here $c$ is a positive constant depending only on $N$ (for example, one can define it by $\left.c^{-1}=2+4 M(1600,2, N)\right)$.

Proof. If $\nu(X)=0$ then the statement is void. If $0<\nu(X)<\infty$ then, by Lemma 3.10, the hypothesis (3.1) of Theorem 3.5 is satisfied, whence the claim follows.

If $\nu(X)=\infty$ then, for given $v$ and $n$, choose a ball $B \subset X$ so big that

$$
v<\nu(B) \text { and } n \leq\left\lceil c \frac{\nu(B)}{v}\right\rceil .
$$

Note that $\nu(B)<\infty$ because $\nu$ is a Radon measure and $B$ is precompact. Applying the previous case to the measure $\nu^{\prime}=\mathbf{1}_{B} \nu$, we obtain a family $\mathcal{A}$ of

$$
\left\lceil c \frac{\nu^{\prime}(X)}{v}\right\rceil=\left\lceil c \frac{\nu(B)}{v}\right\rceil \geq n
$$

annuli satisfying $(a)$ and $(b)$, which was to be proved.

Corollary 3.12. Under the hypotheses of Corollary 3.11, if in addition $0<$ $\nu(X)<\infty$ then, for any positive integer $n$, there exists a family $\mathcal{A}$ of $n$ annuli in $X$ such that

(a) $\nu(A) \geq c \frac{\nu(X)}{n}$ for any $A \in \mathcal{A}$;

(b) the annuli $\{2 A\}_{A \in \mathcal{A}}$ are disjoint.

Here $c$ is the same as in Corollary 3.11. 
Proof. Indeed, setting $v=c \frac{\nu(X)}{n}$ we immediately obtain the claim from Corollary 3.11.

Remark 3.13. In the case $0<\nu(X)<\infty$ the radius $\rho$ from (3.1) satisfies (3.24). Hence, by Remark 3.7, each annulus $A \in \mathcal{A}$ either has the internal radius $\geq \frac{1}{2} V^{-1}(v)$ or is a ball of the radius $\geq \frac{1}{2} V^{-1}(v)$.

\section{Estimating the counting function of an energy form}

In this section we will prove the main estimates of the counting function of an energy form on a pseudometric measure space.

4.1. Main estimate of the counting function. Let $\sigma$ be a signed measure on a topological space $X$, and let $\sigma_{+}$and $\sigma_{-}$be the positive and negative parts of $\sigma$, respectively, so that $\sigma=\sigma_{+}-\sigma_{-}$. The latter means that for any Borel set $A \subset X$ we have $\sigma(A)=\sigma_{+}(A)-\sigma_{-}(A)$, so that $\sigma(A)$ makes sense and takes value in $[-\infty,+\infty]$ provided at least one of the values $\sigma_{+}(A)$ and $\sigma_{-}(A)$ is finite (note that both $\sigma_{+}(A)$ and $\sigma_{-}(A)$ are finite if $\sigma$ is Radon and $A$ is compact). If $\sigma_{+}(A)=\sigma_{-}(A)=+\infty$ then, strictly speaking, $\sigma(A)$ is not defined, but for the sake of simplification of the statements we use here the convention that $\sigma(A)=-\infty$. The same convention we will use for the difference of any two measures.

For any signed measure and for a constant $0 \leq \delta \leq 1$, denote by $\sigma_{\delta}$ the following signed measure:

$$
\sigma_{\delta}:=\delta \sigma_{+}-\sigma_{-} .
$$

Clearly, we have $\left(\sigma_{\delta}\right)_{+}=\delta \sigma_{+}$and $\left(\sigma_{\delta}\right)_{-}=\sigma_{-}$.

Now we can state the main result of this paper.

Theorem 4.1. Let $(X, d)$ be a pseudometric space and $(\mathcal{E}, \mathcal{F})$ be an energy form on $X$. Assume that, for some positive constants $N, Q$, the following conditions hold:

(i) $(X, d)$ satisfies $(2, N)$-covering property;

(ii) the energy form $(\mathcal{E}, \mathcal{F})$ is local and positive definite, and for any ball $B$ in $X$, we have

$$
\operatorname{cap}_{\mathcal{E}}(B, 2 B) \leq Q
$$

Then, for any signed Radon measure $\sigma$ on $X$ such that $\sigma_{+}$is d-non-atomic, we have

$$
N e g(\mathcal{E}-\sigma) \geq\left\lfloor\frac{\sigma_{\delta}(X)}{5 Q}\right\rfloor,
$$

where $\delta \in(0,1)$ depends only on $N$.

We do not claim that the constants 5 and $\delta$ in (4.3) are sharp.

Example 4.2. Let $X$ be the Euclidean space $\mathbb{R}^{n}$ and $\mathcal{E}=\mathcal{E}_{\mu}^{(m)}$ be the $m$-th order energy form defined by (2.49) (where $\mu$ is the Lebesgue measure on $\mathbb{R}^{n}$ ). By (2.60), the hypothesis (4.2) holds provided $n=2 m$. Hence, for any non-atomic Radon measure $\sigma$ on $\mathbb{R}^{n}$, we obtain by Theorem 4.1

$$
\operatorname{Neg}(\mathcal{E}-\sigma) \geq\left\lfloor c \sigma\left(\mathbb{R}^{n}\right)\right\rfloor
$$

where $c>0$ is a constant depending on $n$ and $m$. In fact, one has here a better inequality

$$
\operatorname{Neg}(\mathcal{E}-\sigma) \geq c \sigma\left(\mathbb{R}^{n}\right),
$$


as will follow from Theorem 4.17. Since the generator of the form $\mathcal{E}-\sigma$ is the operator $(-\Delta)^{m}-\sigma$, the above inequalities give also lower bounds for $N e g\left((-\Delta)^{m}-\sigma\right)$.

The proof of Theorem 4.1 will be given in Section 4.4 after a preparatory result in Section 4.2. Here we show what consequences can be deduced from (4.3).

Lemma 4.3. Let $\sigma$ and $\nu$ be two signed measures and $0<\delta \leq 1$. Then the following is true.

(a) $(\sigma+\nu)_{\delta} \geq \sigma_{\delta}+\nu_{\sigma}$;

(b) $\delta \sigma_{\delta} \geq \sigma_{\delta^{2}}$;

(c) If a measurable set $A$ is such that either $\nu_{+}(A)=0$ or $\nu_{-}(A)=0$ then

$$
(\sigma+\nu)_{\delta}(A) \geq\left(\sigma_{\delta^{2}}+\delta \nu\right)(A)
$$

provided the right hand side of (4.4) is non-negative.

Proof. (a) Let us first show that if $\mu_{1}$ and $\mu_{2}$ are two measures then

$$
\left(\mu_{1}-\mu_{2}\right)_{\delta} \geq \delta \mu_{1}-\mu_{2} .
$$

Indeed, set $\mu:=\mu_{1}-\mu_{2}$ and observe that there exists a measure $\alpha$ such that

$$
\mu_{1}=\mu_{+}+\alpha \text { and } \mu_{2}=\mu_{-}+\alpha .
$$

Then

$$
\delta \mu_{1}-\mu_{2}=\delta\left(\mu_{+}+\alpha\right)-\left(\mu_{-}+\alpha\right)=\mu_{\delta}+(\delta-1) \alpha \leq \mu_{\delta},
$$

that is (4.5).

Applying (4.5) to $\mu_{1}=\sigma_{+}+\nu_{+}$and $\mu_{2}=\sigma_{-}+\nu_{-}$, we obtain

$$
(\sigma+\nu)_{\delta} \geq \delta\left(\sigma_{+}+\nu_{+}\right)-\left(\sigma_{-}+\nu_{-}\right)=\sigma_{\delta}+\nu_{\delta},
$$

which was to be proved.

(b) We have

$$
\delta \sigma_{\delta}=\delta^{2} \sigma_{+}-\delta \sigma_{-} \geq \delta^{2} \sigma_{+}-\sigma_{-}=\sigma_{\delta^{2}} .
$$

(c) By part $(a)$ we have

$$
(\sigma+\nu)_{\delta}(A) \geq \sigma_{\delta}(A)+\delta \nu_{+}(A)-\nu_{-}(A) .
$$

If $\nu_{-}(A)=0$ then

$$
(\sigma+\nu)_{\delta}(A) \geq \sigma_{\delta}(A)+\delta \nu(A) \geq\left(\sigma_{\delta^{2}}+\delta \nu\right)(A) .
$$

If $\nu_{+}(A)=0$ then

$$
(\sigma+\nu)_{\delta}(A) \geq \sigma_{\delta}(A)-\nu_{-}(A)=\sigma_{\delta}(A)+\nu(A) .
$$

By hypothesis, we have

$$
\sigma_{\delta^{2}}(A)+\delta \nu(A) \geq 0,
$$

which implies by part $(b)$ than

$$
\delta \sigma_{\delta}(A)+\delta \nu(A) \geq 0 .
$$

Hence, $\sigma_{\delta}(A)+\nu(A) \geq 0$ whence, by $\delta \leq 1$,

$$
\sigma_{\delta}(A)+\nu(A) \geq \delta \sigma_{\delta}(A)+\delta \nu(A) \geq \sigma_{\delta^{2}}(A)+\delta \nu(A) .
$$

Substituting into (4.6) we finish the proof. 
Lemma 4.4. Let $(\mathcal{E}, \mathcal{F})$ be an energy form on a pseudometric space $(X, d)$, and assume that, for any signed Radon measure $\sigma$ on $X$ such that $\sigma_{+}$is $d$-non-atomic,

$$
N e g(\mathcal{E}-\sigma) \geq\left\lfloor\frac{\sigma_{\delta}(X)}{C}\right\rfloor,
$$

where $0<\delta \leq 1$ and $C>0$ are some constant. If $\mu$ is a $d$-non-atomic measure on $X$ then, for all real $\lambda$,

$$
\mathcal{N}_{\lambda}(\mathcal{E}-\sigma, \mu) \geq\left\lfloor\frac{\sigma_{\delta^{2}}(X)+\delta \lambda \mu(X)}{C}\right\rfloor .
$$

If $0<\mu(X)<\infty$ then, for any $k=1,2, \ldots$,

$$
\lambda_{k}(\mathcal{E}-\sigma, \mu) \leq \frac{C k-\sigma_{\delta^{2}}(X)}{\delta \mu(X)} .
$$

Proof. Set $\widetilde{\sigma}=\sigma+\lambda \mu$. Then $\widetilde{\sigma}$ is a signed Radon measure on $X$, and $\widetilde{\sigma}_{+}$is $d$-non-atomic. By (2.41) we have

$$
\mathcal{N}_{\lambda}(\mathcal{E}-\sigma, \mu)=\operatorname{Neg}(\mathcal{E}-\tilde{\sigma}) .
$$

Applying (4.3) to $\widetilde{\sigma}$ instead of $\sigma$, we obtain

$$
\mathcal{N}_{\lambda}(\mathcal{E}-\sigma, \mu) \geq\left\lfloor\frac{(\sigma+\lambda \mu)_{\delta}(X)}{C}\right\rfloor .
$$

If $\sigma_{\delta^{2}}(X)+\delta \lambda \mu(X) \leq 0$ then (4.8) holds trivially. Otherwise, we have by Lemma 4.3 ,

$$
(\sigma+\lambda \mu)_{\delta} \geq \sigma_{\delta^{2}}(X)+\delta \lambda \mu(X)
$$

whence (4.8) follows. Finally, (4.9) follows from (4.8) by Lemma 2.8.

Remark 4.5. Hence, the estimates (4.8) and (4.9) are true in the setting of Theorem 4.1 with $C=5 Q$. It is not known yet whether the constant $\delta$ in Theorem 4.1 can be taken $\delta=1$. If so then $\sigma_{\delta}(X)$ and $\sigma_{\delta^{2}}(X)$ could be replaced by $\sigma(X)$, which would be most convenient for applications. If $\sigma \geq 0$ or $\sigma \leq 0$ then this can be achieved by changing the other constants. For example, if $\sigma \geq 0$ then $\sigma_{\delta^{2}}=\delta^{2} \sigma$ and (4.8) becomes

$$
\mathcal{N}_{\lambda}(\mathcal{E}-\sigma, \mu) \geq\left\lfloor\frac{\delta \sigma(X)+\lambda \mu(X)}{C^{\prime}}\right\rfloor,
$$

where $C^{\prime}=C / \delta$. Similarly, (4.9) becomes

$$
\lambda_{k}(\mathcal{E}-\sigma, \mu) \leq \frac{C^{\prime} k-\delta \sigma(X)}{\mu(X)} .
$$

It would be very useful to prove (4.11) and/or (4.12) also for a signed measure $\sigma$. In Section 5.5 we prove (4.12) for a signed measure $\sigma$ assuming in addition that $\mathcal{E}$ is a Riemannian energy form and the perturbed form $\mathcal{E}-\sigma$ is positive definite. We conjecture that (4.11) and (4.12) are true in general, without these assumptions.

Remark 4.6. If the floor function in (4.7) is replaced by the ceiling function (as will be in Theorem 4.17 below) then the floor function in (4.8) is also replaced by the ceiling function, and the term $C k$ in the right hand side of (4.9) is replaced by $C(k-1)$. 
4.2. Decomposition of a pseudometric space by capacitors. We say that a sequence $\left\{\left(F_{i}, G_{i}\right)\right\}_{i=1}^{n}$ of capacitors is disjoint if the sequence of sets $\left\{G_{i}\right\}_{i=1}^{n}$ is disjoint. The following result plays the crucial role in the proof of Theorem 4.1.

Theorem 4.7. Let $(X, d)$ be a pseudometric space, $\nu$ be a Radon measure on $X$, and $(\mathcal{E}, \mathcal{F})$ be an energy form on $X$. Assume that the following hypotheses are satisfied, for some positive constants $N, Q$ :

(i) $(X, d)$ satisfies $(2, N)$-covering property;

(ii) measure $\nu$ is d-non-atomic;

(iii) the energy form $(\mathcal{E}, \mathcal{F})$ is positive definite and, for any ball $B$ in $X$,

$$
\operatorname{cap}_{\mathcal{E}}(B, 2 B) \leq Q
$$

Then, for any $0<v<\nu(X)$ and any positive integer $n$ such that

$$
n \leq\left\lceil c \frac{\nu(X)}{v}\right\rceil,
$$

there exist $n$ disjoint capacitors $\left(F_{i}, G_{i}\right)$ in $X$ such that, for all $i=1,2, \ldots, n$,

$$
\nu\left(F_{i}\right) \geq v
$$

and

$$
\operatorname{cap}_{\mathcal{E}}\left(F_{i}, G_{i}\right) \leq 4 Q \text {. }
$$

Here $c$ is a positive constant depending only on $N$.

A version of this Theorem was proved in [27, Theorem 1] using an abstract version of Korevaar's argument [37]. The present Theorem 4.7 needs fewer assumptions about the energy form, unlike [27, Theorem 1], which required the energy form to satisfy the Markov property.

Proof. The finiteness of $\operatorname{cap}_{\mathcal{E}}(B, 2 B)$ implies that the ball $B$ is precompact. Indeed, the class $\mathcal{T}(B, 2 B)$ is non-empty, let $f \in \mathcal{T}(B, 2 B)$. Since supp $f$ is compact and $\left.f\right|_{B}=1$, it follows that $B \subset \operatorname{supp} f$ and $B$ is precompact.

Hence, all the hypotheses of Corollary 3.11 are satisfied. By Corollary 3.11, there exists a sequence $\left\{F_{i}\right\}_{i=1}^{n}$ of $n$ annuli such that $\nu\left(F_{i}\right) \geq v$ and the annuli $G_{i}:=2 F_{i}$ are disjoint.

We are left to verify (4.16). Indeed, let $F_{i}=A(x, r, R)$ and, hence, $G_{i}=$ $A(x, r / 2,2 R)$. Let us write for simplicity $B_{s}=B(x, s)$. If $r=0$ then by (4.13) we have

$$
\operatorname{cap}_{\mathcal{E}}\left(F_{i}, G_{i}\right)=\operatorname{cap}_{\mathcal{E}}\left(B_{R}, B_{2 R}\right) \leq Q .
$$

If $r>0$ then, by Lemma 2.3 and (4.13), we obtain

$$
\begin{aligned}
\operatorname{cap}_{\mathcal{E}}\left(F_{i}, G_{i}\right)^{1 / 2} & =\operatorname{cap}_{\mathcal{E}}\left(B_{R} \backslash B_{r}, B_{2 R} \backslash B_{r / 2}\right)^{1 / 2} \\
& \leq \operatorname{cap}_{\mathcal{E}}\left(B_{r / 2}, B_{r}\right)^{1 / 2}+\operatorname{cap}_{\mathcal{E}}\left(B_{R}, B_{2 R}\right)^{1 / 2} \\
& \leq 2 Q^{1 / 2}
\end{aligned}
$$

whence (4.16) follows.

Remark 4.8. Let $0<\nu(X)<\infty$. As follows from Remark 3.13, for each annulus $F_{i}=A(x, r, R)$ we have either $r \geq \frac{1}{2} V^{-1}(v)$ or $r=0$ and $R \geq \frac{1}{2} V^{-1}(v)$, where $V^{-1}$ is the generalized inverse to the function $V(r)$ defined by (3.23). Therefore, as we see from the above proof, the hypothesis (4.13) can be restricted to the balls of radii $\geq \frac{1}{4} V^{-1}(v)$. 
Corollary 4.9. Under the hypotheses of Theorem 4.7, if $0<\nu(X)<\infty$ then, for any positive integer $n$, there exist $n$ disjoint capacitors $\left(F_{i}, G_{i}\right)$ such that, for all $i=1,2, \ldots, n$,

$$
\nu\left(F_{i}\right) \geq c \frac{\nu(X)}{n}
$$

and

$$
\operatorname{cap}_{\mathcal{E}}\left(F_{i}, G_{i}\right) \leq 4 Q,
$$

where $c$ is the same as in Theorem 4.7 .

Proof. Indeed, given a positive integer $n$, define $v$ by

$$
v=c \frac{\nu(X)}{n},
$$

where $c$ is the constant from Theorem 4.7. Then $0<v<\nu(X)$, and the conclusion follows from Theorem 4.7.

Remark 4.10. As follows from Remark 4.8, the hypothesis (4.13) can be restricted to the balls of radii $\geq \frac{1}{4} V^{-1}\left(\frac{c \nu(X)}{n}\right)$, where $V(r)$ defined by (3.23).

To show an example of application of Corollary 4.9, let us give a direct proof of the estimate (4.9) in the case $\sigma=0$ (without using Theorem 4.1), which contains the main idea of the proof of Theorem 4.1.

Corollary 4.11. Let $(X, d)$ be a pseudometric space, $\nu$ be a Radon measure on $X$, and $(\mathcal{E}, \mathcal{F})$ be an energy form on $X$. Assume that, for some positive constants $N, Q$, the following conditions hold:

(i) $(X, d)$ satisfies $(2, N)$-covering property;

(ii) measure $\nu$ is a $d$-non-atomic and $0<\nu(X)<\infty$;

(iii) the energy form $(\mathcal{E}, \mathcal{F})$ is local and positive definite, and for any ball $B$ in $X$, we have

$$
\operatorname{cap}_{\mathcal{E}}(B, 2 B) \leq Q
$$

Then, for any $k=1,2, \ldots$,

$$
\lambda_{k}(\mathcal{E}, \nu) \leq C Q \frac{k}{\nu(X)}
$$

where the positive constant $C$ depends only on $N$.

Proof. By Corollary 4.9, there exists $k$ disjoint capacitors $\left(F_{i}, G_{i}\right)$ satisfying (4.17) and (4.18). For any $\varepsilon>0$ there exists a test function $f_{i} \in \mathcal{T}\left(F_{i}, G_{i}\right)$ such that

$$
\mathcal{E}\left[f_{i}\right]<\operatorname{cap}_{\mathcal{E}}\left(F_{i}, G_{i}\right)+\varepsilon \leq 4 Q+\varepsilon .
$$

Take $\varepsilon=Q$ and fix such a function $f_{i}$. Since $\left.f_{i}\right|_{F_{i}}=1$, we have

whence

$$
\nu\left[f_{i}\right] \geq \nu\left(F_{i}\right) \geq c \frac{\nu(X)}{k},
$$

where $C:=5 / c$.

$$
\frac{\mathcal{E}\left[f_{i}\right]}{\nu\left[f_{i}\right]}<\frac{5 Q}{c \frac{\nu(X)}{k}}=C Q \frac{k}{\nu(X)}=: \lambda,
$$


Hence, for any $f=f_{i}$ we have

$$
\mathcal{E}[f]<\lambda \nu[f] .
$$

Since the supports of the functions $f_{i}$ are disjoint and the form $\mathcal{E}$ is local, we conclude that (4.21) holds for any function $f \in \operatorname{span}\left(f_{1}, \ldots, f_{n}\right)$ except for $f=0$. By the definition of the counting function, we obtain $\mathcal{N}_{\lambda}(\mathcal{E}, \nu) \geq k$, whereas

$$
\mathcal{N}_{\lambda_{k}}(\mathcal{E}, \nu)=\sup \left\{n: \lambda_{n}<\lambda_{k}\right\} \leq k-1 .
$$

Therefore, $\lambda_{k} \leq \lambda$ which was to be proved.

Remark 4.12. As follows from Remark 4.10, in order to obtain (4.20) for a fixed index $k$, it suffices to assume the hypothesis (4.19) only for the balls of radii at least $\frac{1}{4} V^{-1}\left(\frac{c \nu(X)}{k}\right)$.

Example 4.13. Let $X$ be the Euclidean space $\mathbb{R}^{n}, \mu$ be the Lebesgue measure on $\mathbb{R}^{n}$, and $\mathcal{E}=\mathcal{E}_{\mu}^{(m)}$ be the $m$-th order energy form on $\mathbb{R}^{n}$ defined by $(2.49)$. If $n=2 m$ then the capacity of the form $\mathcal{E}$ satisfies (4.19) as follows from (2.60). Let $q$ be a smooth positive function on $\mathbb{R}^{n}$, and consider the measure $\nu$ defined by $d \nu=q d \mu$. A generator of the form $\mathcal{E}$ is the operator $\frac{1}{q}(-\Delta)^{m}$. We obtain from Corollary 4.11 that if $2 m=n$ and

$$
\nu\left(\mathbb{R}^{n}\right)=\int_{\mathbb{R}^{n}} q d \mu<\infty
$$

then

$$
\lambda_{k}\left(\frac{1}{q}(-\Delta)^{m}\right) \leq C \frac{k}{\nu\left(\mathbb{R}^{n}\right)} .
$$

4.3. Eigenvalues on fractal spaces. Let us show how Corollary 4.11 can be used in conjunction with Remark 4.12 to handle the case when the capacity uniform bound (4.19) is not available but, instead, one has (2.58) and (2.59). The next statement applies to most fractal spaces.

Corollary 4.14. Let $(X, d)$ be a pseudometric space, $\nu$ be a Radon measure on $X$, and $(\mathcal{E}, \mathcal{F})$ be an energy form on $X$. Assume that, for some positive constants $C_{1}, C_{2}, N$ the following conditions hold:

(i) $(X, d)$ satisfies $(2, N)$-covering property;

(ii) measure $\nu$ is a d-non-atomic, $0<\nu(X)<\infty$, and, for any ball $B(x, r)$ in $X$,

$$
\nu(B(x, r)) \leq C_{1} r^{\alpha},
$$

where $\alpha>0$;

(iii) the energy form $(\mathcal{E}, \mathcal{F})$ is local and positive definite, and, for any ball $B(x, r)$ in $X$, we have

$$
\operatorname{cap}_{\mathcal{E}}(B(x, r), B(x, 2 r)) \leq C_{2} r^{\alpha-\beta},
$$

where $\beta \geq \alpha$;

Then, for all $k=1,2, \ldots$,

$$
\lambda_{k}(\mathcal{E}, \nu) \leq C\left(\frac{k}{\nu(X)}\right)^{\beta / a}
$$

where the constant $C$ depends on $N, C_{1}, C_{2}, \alpha, \beta$. 
Remark 4.15. In the case $\alpha=\beta$ the hypothesis (4.23) becomes (4.19) and hence, by Corollary 4.11, the hypothesis (4.22) can be omitted. In the case $\alpha \neq \beta$ the hypothesis (4.22) is essential because the value of $\alpha-\beta$ from (4.23) does not allow to recover the value of $\beta / \alpha$ necessary for (4.24). It is not clear whether the condition $\beta \geq \alpha$ can be dropped here. whence

Proof. By (4.22) and by definition (3.23) of $V(r)$, we have $V(r) \leq C_{1} r^{\alpha}$

$$
V^{-1}(v) \geq\left(v / C_{1}\right)^{1 / \alpha} .
$$

Fix an index $k$. If $r \geq \frac{1}{4} V^{-1}\left(\frac{c \nu(X)}{k}\right)$ (where $c>0$ is the constant from Corollary 4.11) then

$$
r \geq \frac{1}{4}\left(\frac{c \nu(X)}{C_{1} k}\right)^{1 / \alpha}
$$

Therefore, using $\alpha-\beta \leq 0$ and (4.23), we obtain, for any ball $B$ of such a radius $r$,

$$
\operatorname{cap}_{\mathcal{E}}(B, 2 B) \leq C_{2} r^{\alpha-\beta} \leq C_{2}\left(\frac{1}{4}\left(\frac{c \nu(X)}{C_{1} k}\right)^{1 / \alpha}\right)^{\alpha-\beta}=C^{\prime}\left(\frac{k}{\nu(X)}\right)^{\beta / \alpha-1}=: Q
$$

By Remark 4.12, we can apply Corollary 4.11 and, hence, obtain by (4.20)

$$
\lambda_{k}(\mathcal{E}, \nu) \leq C Q \frac{k}{\nu(X)}=C C^{\prime}\left(\frac{k}{\nu(X)}\right)^{\beta / \alpha},
$$

which was to be proved.

4.4. Proof of the main estimate. Here we prove Theorem 4.1, that is the following estimate

$$
N e g(\mathcal{E}-\sigma) \geq\left\lfloor\frac{\sigma_{\delta}(X)}{5 Q}\right\rfloor=: k .
$$

If $k \leq 0$ then there is nothing to do, so we assume in the sequel that $k>0$. In particular, this implies $\sigma_{\delta}(X)>0$ and hence $\sigma_{-}(X)<\infty$.

Without loss of generality we can assume that also $\sigma_{+}(X)<\infty$. Indeed, if $\sigma_{+}(X)=\infty$ then consider a signed measure

$$
\sigma^{(r)}:=\mathbf{1}_{B(x, r)} \sigma_{+}-\sigma_{-},
$$

where $x \in X$ is fixed and $r>0$. Hypothesis (4.2) implies that the ball $B(x, r)$ is precompact (see the proof of Theorem 4.7). Since the measure $\sigma_{+}$is Radon, we see that

$$
\left|\sigma^{(r)}\right|(X)=\sigma_{+}(B(x, r))+\sigma_{-}(X)<\infty .
$$

If we can prove that

$$
N e g\left(\mathcal{E}-\sigma^{(r)}\right) \geq\left\lfloor\frac{\sigma_{\delta}^{(r)}(X)}{5 Q}\right\rfloor
$$

then passing to the limit as $r \rightarrow \infty$ and using

$$
\operatorname{Neg}(\mathcal{E}-\sigma) \geq N e g\left(\mathcal{E}-\sigma^{(r)}\right)
$$

we will obtain (4.25). Hence, we can assume in the sequel that $|\sigma|(X)<\infty$.

In order to prove (4.25) it suffices to construct $k$ linearly independent functions $f_{1}, f_{2}, \ldots, f_{k}$ in $\mathcal{F}$ such that

$$
\mathcal{E}[f]-\sigma[f]<0
$$


for any $f \in \operatorname{span}\left\{f_{i}\right\} \backslash\{0\}$. The functions $f_{i}$ in our construction will have disjoint supports. Hence, by the locality of $\mathcal{E}-\sigma$, we have $(\mathcal{E}-\sigma)\left(f_{i}, f_{j}\right)=0$ for all $i \neq j$, so it suffices to establish (4.26) for $f=f_{i}, i=1,2, \ldots, k$.

Suppose that we have $k$ disjoint capacitors $\left(F_{i}, G_{i}\right)$ in $X$ of finite capacity. Then we choose $f_{i} \in \mathcal{T}\left(F_{i}, G_{i}\right)$ to be a nearly optimal test function in the sense that

$$
\mathcal{E}\left[f_{i}\right]<\operatorname{cap}_{\mathcal{E}}\left(F_{i}, G_{i}\right)+\varepsilon,
$$

where $\varepsilon>0$ will be specified later on (see Fig. 10).

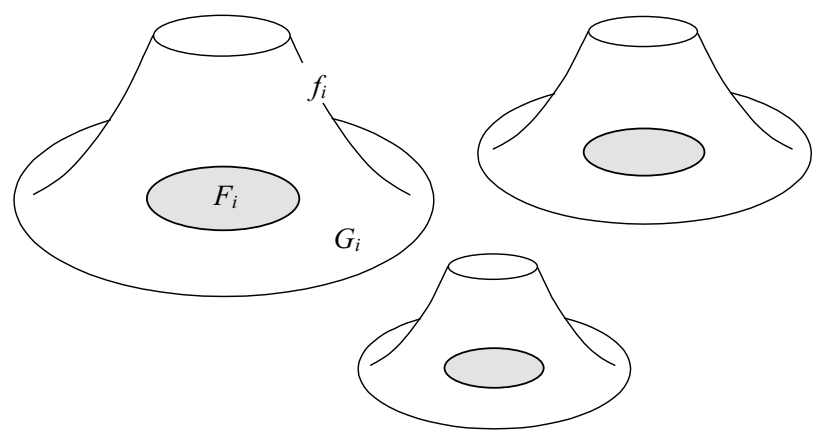

Figure 10. Capacitors $\left(F_{i}, G_{i}\right)$ and their test functions.

By the definition of $\mathcal{T}\left(F_{i}, G_{i}\right)$, we have $f_{i} \in \mathcal{F} \cap C_{0}\left(G_{i}\right), f_{i}=1$ on $F_{i}$, and $0 \leq f_{i} \leq 1$. Therefore,

$$
\sigma_{-}\left[f_{i}\right] \leq \sigma_{-}\left(G_{i}\right) \quad \text { and } \quad \sigma_{+}\left[f_{i}\right] \geq \sigma_{+}\left(F_{i}\right) .
$$

Then (4.26) will follow if we know that

$$
\operatorname{cap}_{\mathcal{E}}\left(F_{i}, G_{i}\right)+\varepsilon+\sigma_{-}\left(G_{i}\right) \leq \sigma_{+}\left(F_{i}\right) .
$$

The assumption $\sigma_{\delta}(X)>0$ implies $\sigma_{+}(X)>0$. Hence, the metric-measure space $\left(X, d, \sigma_{+}\right)$with the form $(\mathcal{E}, \mathcal{F})$ satisfies all the hypotheses of Corollary 4.9. By Corollary 4.9 , for any $k=1,2, \ldots$, there exists $n=2 k$ disjoint capacitors $\left(F_{i}, G_{i}\right)$ on $X$ such that

$$
\sigma_{+}\left(F_{i}\right) \geq c \frac{\sigma_{+}(X)}{2 k} \text { and } \operatorname{cap}_{\mathcal{E}}\left(F_{i}, G_{i}\right) \leq 4 Q
$$

Since

$$
\sum_{i=1}^{2 k} \sigma_{-}\left(G_{i}\right) \leq \sigma_{-}(X),
$$

there are at most $k$ sets $G_{i}$ for which

$$
\sigma_{-}\left(G_{i}\right)>\frac{\sigma_{-}(X)}{k}
$$

and, hence, there are at least $n-k=k$ sets $G_{i}$, for which

$$
\sigma_{-}\left(G_{i}\right) \leq \frac{\sigma_{-}(X)}{k} .
$$


We can assume that these sets are $G_{i}, i=1,2, \ldots, k$. Substituting (4.28) and (4.29) into (4.27) and choosing $\varepsilon=Q$, we see that (4.27) will be satisfied for capacitors $\left(F_{i}, G_{i}\right), i=1,2, \ldots, k$, provided

$$
5 Q+\frac{\sigma_{-}(X)}{k} \leq c \frac{\sigma_{+}(X)}{2 k} .
$$

Set $\delta:=c / 2$ and observe that (4.30) is equivalent to

$$
k \leq \frac{\delta \sigma_{+}(X)-\sigma_{-}(X)}{5 Q}=\frac{\sigma_{\delta}(X)}{5 Q},
$$

which is true by the choice of $k$.

Remark 4.16. In the case $|\sigma|(X)<\infty$, by Remark 4.10, the hypothesis (4.2) of Theorem 4.1 can be assumed only for the balls of radii $\geq \frac{1}{4} V^{-1}\left(c \frac{\sigma_{+}(X)}{2 k}\right)$ where $k=\left\lfloor\frac{\sigma_{\delta}(X)}{5 Q}\right\rfloor$ and $V^{-1}$ is the generalized inverse to the function

$$
V(r)=\sup _{x \in X} \sigma_{+}(B(x, r)) .
$$

4.5. Strongly local energy forms. In this section, we slightly improve the estimate of Theorem 4.1 for strongly local energy forms.

Theorem 4.17. Suppose that, under the hypotheses of Theorem 4.1 the energy form $(\mathcal{E}, \mathcal{F})$ is strongly local. Then the estimate (4.3) in the conclusion of Theorem 4.1 can be replaced by

$$
N e g(\mathcal{E}-\sigma) \geq\left\lceil\frac{\sigma_{\delta}(X)}{10 Q}\right\rceil
$$

The point of this theorem is that the floor function in the estimate (4.3) can replaced by the ceiling function as in (4.32) This improvement is only noticeable when, for example, $0<\frac{\sigma_{\delta}(X)}{10 Q}<1$. In this case, the estimate (4.3) becomes trivial whereas (4.32) still gives $N e g(\mathcal{E}-\sigma) \geq 1$. The strong locality hypothesis is essential for that, as it will be shown in the example at the end of this section.

Similarly to Lemma 4.4 (cf. Remark 4.6), the estimate (4.32) implies the following. If $\mu$ is a $d$-non-atomic Radon measure on $X$ then, for all real $\lambda$,

$$
\mathcal{N}_{\lambda}(\mathcal{E}-\sigma, \mu) \geq\left\lceil\frac{\sigma_{\delta^{2}}(X)+\delta \lambda \mu(X)}{10 Q}\right\rceil .
$$

If in addition $0<\mu(X)<\infty$ then, for any $k=1,2, \ldots$,

$$
\lambda_{k}(\mathcal{E}-\sigma, \mu) \leq \frac{10 Q(k-1)-\sigma_{\delta^{2}}(X)}{\delta \mu(X)} .
$$

The next lemma will be used in the proof of Theorem 4.17.

Lemma 4.18. Let $(\mathcal{E}, \mathcal{F})$ be a strongly local, positive definite energy form on a pseudometric space $(X, d)$. Assume that, for any ball $B$ in $X$,

$$
\operatorname{cap}_{\mathcal{E}}(B, 2 B) \leq Q,
$$

where $Q$ is a positive constant. Then, for any signed Radon measure $\sigma$ on $X$ such that $\sigma(X)>0$, we have

$$
N e g(\mathcal{E}-\sigma) \geq 1
$$


Proof. The hypothesis $\sigma(X)>0$ implies, in particular, that $\sigma_{-}(X)<\infty$. By the same argument as in the proof of Theorem 4.1, we can assume that also $\sigma_{+}(X)<\infty$.

Consider a sequence of balls $B_{n}=B\left(x, 2^{n}\right)$ where $x$ is a fixed reference point in $X$. By hypothesis (4.35), we have

$$
\operatorname{cap}_{\mathcal{E}}\left(B_{n}, B_{n+1}\right) \leq Q
$$

By Lemma 2.5, for all indices $n<m$,

$$
\operatorname{cap}_{\mathcal{E}}\left(B_{n}, B_{m}\right)^{-1} \geq \sum_{i=n}^{m-1} \operatorname{cap}_{\mathcal{E}}\left(B_{i}, B_{i+1}\right)^{-1} \geq(m-n) Q^{-1}
$$

whence

$$
\operatorname{cap}_{\mathcal{E}}\left(B_{n}, B_{m}\right) \leq \frac{Q}{m-n}
$$

Let $f \in \mathcal{T}\left(B_{n}, B_{m}\right)$ be such that

$$
\mathcal{E}[f]<\frac{2 Q}{m-n} .
$$

Using the fact that $0 \leq f \leq 1$ and $f=1$ on $B_{n}$, we obtain

$$
\begin{aligned}
\mathcal{E}[f]-\sigma[f] & <\frac{2 Q}{m-n}-\sigma\left(B_{n}\right)-\int_{X \backslash B_{n}} f^{2} d \sigma \\
& \leq \frac{2 Q}{m-n}-\sigma\left(B_{n}\right)+|\sigma|\left(X \backslash B_{n}\right) .
\end{aligned}
$$

Since $|\sigma|(X)<\infty$, for large enough $n$ and $m$ we obtain

$$
(\mathcal{E}-\sigma)[f]<-\sigma(X)+\varepsilon,
$$

where $\varepsilon>0$ is prescribed. Choosing $\varepsilon<\sigma(X) / 2$, we conclude $(\mathcal{E}-\sigma)[f]<0$ whence (4.36) follows.

Proof of Theorem 4.17. We need to prove (4.32), that is

$$
N e g(\mathcal{E}-\sigma) \geq\left\lceil\frac{1}{2} \kappa\right\rceil,
$$

where $\kappa=\frac{\sigma_{\delta}(X)}{5 Q}$. If $\kappa \leq 0$ then there is nothing to do, so assume $\kappa>0$. In this case $\sigma(X)>0$ and hence, by Lemma 4.18,

$$
\operatorname{Neg}(\mathcal{E}-\sigma) \geq 1 \text {. }
$$

On the other hand, by Theorem 4.1,

$$
\operatorname{Neg}(\mathcal{E}-\sigma) \geq\lfloor\kappa\rfloor .
$$

Then (4.39) follows by the elementary inequality

$$
\max (\lfloor\kappa\rfloor, 1) \geq\left\lceil\frac{1}{2} \kappa\right\rceil .
$$


Example 4.19. As in Example 4.13, let $X=\mathbb{R}^{n}, \mu$ be the Lebesgue measure on $\mathbb{R}^{n}$, and $\mathcal{E}=\mathcal{E}_{\mu}^{(m)}$ be the $m$-th order energy form on $\mathbb{R}^{n}$ defined by (2.49). Assume that $n=2 m$ so that the form $\mathcal{E}$ satisfies (4.35). Let $q$ be a non-negative $L_{\text {loc }}^{1}$-function in $\mathbb{R}^{n}$. Considering the measure $\sigma$ defined by $d \sigma=q d \mu$, we obtain by (4.32) that

$$
N e g\left((-\Delta)^{m}-q\right) \geq c \int_{\mathbb{R}^{n}} q d \mu
$$

where $c=c(n)>0$.

Example 4.20. Let us show that if the form $\mathcal{E}$ is local but not strongly local it can happen that $\sigma \geq 0, \sigma(X)>0$ but $N e g(\mathcal{E}-\sigma)=0$. Indeed, take $X=\mathbb{R}^{2}$ with the Euclidean distance $d$, and consider the form $\mathcal{E}=\mathcal{E}_{\mu}+\nu$ with domain $\operatorname{Lip}_{0}\left(\mathbb{R}^{2}\right)$, that is

$$
\mathcal{E}(f, g)=\int_{\mathbb{R}^{2}} \nabla f \cdot \nabla g d \mu+\int_{\mathbb{R}^{2}} f g d \nu
$$

where $\mu$ is the Lebesgue measure on $\mathbb{R}^{2}$ and $\nu$ is a measure on $\mathbb{R}^{2}$ such that

$$
0<\nu\left(\mathbb{R}^{2}\right)<\infty .
$$

It is easy to see that, for any capacitor $(F, G)$ in $\mathbb{R}^{2}$,

$$
\operatorname{cap}_{\mathcal{E}}(F, G) \leq \operatorname{cap}_{\mathcal{E}_{\mu}}(F, G)+\nu(G)
$$

whence it follows that, for any ball $B$,

$$
\operatorname{cap}_{\mathcal{E}}(B, 2 B) \leq \operatorname{cap}_{\mathcal{E}_{\mu}}(B, 2 B)+\nu\left(\mathbb{R}^{2}\right)=\text { const. }
$$

Therefore, all the hypotheses of Theorem 4.1 are satisfied for the form $\mathcal{E}$. However, the claim of Lemma 4.18 (and that of Theorem 4.17) is not true in this case. Indeed, just take $\sigma=\nu$ so that $\mathcal{E}-\sigma=\mathcal{E}_{\mu}$. Then $\sigma\left(\mathbb{R}^{2}\right)>0$ but $N e g(\mathcal{E}-\sigma)=\operatorname{Neg}\left(\mathcal{E}_{\mu}\right)=$ 0 .

Let us show that the hypothesis (4.35) is also essential for Lemma 4.18. For that, consider $X=\mathbb{R}^{3}$ with the standard form $\mathcal{E}_{\mu}$, for which (4.35) does not hold. The form $\mathcal{E}_{\mu}$ is strongly local, but nevertheless there exists a positive measure $\sigma$ in $\mathbb{R}^{3}$ such that $\operatorname{Neg}\left(\mathcal{E}_{\mu}-\sigma\right)=0$. For example, this is the case whenever $\sigma$ satisfies the estimate

because of the Hardy inequality

$$
\frac{d \sigma}{d \mu}(x) \leq \frac{1}{4|x|^{2}},
$$

$$
\int_{\mathbb{R}^{3}} \frac{1}{4|x|^{2}} f^{2}(x) d \mu(x) \leq \int_{\mathbb{R}^{3}}|\nabla f|^{2} d \mu,
$$

which is true for any $f \in C_{0}^{\infty}\left(\mathbb{R}^{3}\right)$ (see, for example, [50, Section X.2]).

\section{Eigenvalues on Riemannian manifolds}

Let $X$ be a Riemannian manifold and $d_{0}$ be the geodesic distance on $X$ (note that $d_{0}$ may take value $\infty$ if $X$ is disconnected).

Definition 5.1. A pseudometric $d$ on $X$ is called Riemannian if $d$ is dominated by $d_{0}$, that is

$$
d(x, y) \leq d_{0}(x, y) \quad \text { for all } x, y \in X
$$

The condition (5.1) and the triangle inequality imply that, for any $x \in X$, the function $y \mapsto d(x, y)$ is locally Lipschitz and $|\nabla d(x, \cdot)| \leq 1$. 
Definition 5.2. A pseudometric space $(X, d)$ is called Riemannian if $X$ is a Riemannian manifold and $d$ is a Riemannian pseudometric on $X$.

For example, if $X$ is a connected Riemannian manifold then $\left(X, d_{0}\right)$ is a Riemannian (pseudo)metric space. Let $T: X \rightarrow X^{\prime}$ be an isometric immersion of a Riemannian manifold $X$ into a connected Riemannian manifold $X^{\prime}$, and let $d^{\prime}$ be the geodesic distance on $X^{\prime}$. Then the identity

$$
d(x, y)=d^{\prime}(T(x), T(y))
$$

defines the extrinsic metric on $X$, which obviously is a Riemannian pseudometric. Hence, $(X, d)$ is a Riemannian pseudometric space.

In this section, we adapt the results of the previous sections to a Riemannian pseudometric space $(X, d)$. As before, we denote by $B(x, r)$ the balls of the pseudometric $d$. Set $\mathcal{F}=\operatorname{Lip}_{0}(X)$ and recall that any Radon measure $\mu$ on $X$ induces a strongly local positive definite energy form $\left(\mathcal{E}_{\mu}, \mathcal{F}\right)$ on the weighted manifold $(X, \mu)$ as follows:

$$
\mathcal{E}_{\mu}(f, g)=\int_{X} \nabla f \cdot \nabla g d \mu
$$

\subsection{Quadratic volume growth.}

Theorem 5.3. Let $(X, d)$ be a Riemannian pseudometric space, $\mu$ be a Radon measure on $X$, and $\mathcal{E}=\mathcal{E}_{\mu}$. Assume that the following properties are satisfied, for some positive constants $M, N$ :

(a) space $(X, d)$ satisfies $(2, N)$-covering property;

(b) all balls in $(X, d)$ are precompact;

(c) for all $x \in X$ and $r>0$

$$
\mu(B(x, r)) \leq M r^{2} .
$$

Then, for any signed Radon measure $\sigma$ on $X$ such that $\sigma_{+}$is d-non-atomic,

$$
N e g(\mathcal{E}-\sigma) \geq \frac{\sigma_{\delta}(X)}{100 M}
$$

where $\delta \in(0,1)$ depends only on $N$.

The estimate (5.5) implies, by Lemma 4.4, that for any $d$-non-atomic Radon measure $\nu$ on $X$ and for any real $\lambda$,

$$
\mathcal{N}_{\lambda}(\mathcal{E}-\sigma, \nu) \geq \frac{\sigma_{\delta^{2}}(X)+\delta \lambda \nu(X)}{100 M}
$$

If $0<\nu(X)<\infty$ then for all $k=1,2, \ldots$,

$$
\lambda_{k}(\mathcal{E}-\sigma, \nu) \leq \frac{C(k-1)-\sigma_{\delta^{2}}(X)}{\nu(X)}
$$

where $C=C(N)$. In particular, these estimate hold for $\nu=\mu$ because $\mu$ is $d$-nonatomic by (5.4).

Proof. Let us show that (5.4) implies

$$
\operatorname{cap}_{\mathcal{E}}(B, 2 B) \leq 11 M \text {. }
$$


Fix a point $x \in X$ and denote for simplicity $B_{r}=B(x, r)$ and $V_{r}=\mu(B(x, r))$. For all $0<r<R$, the following inequality is always true:

$$
\operatorname{cap}_{\mathcal{E}}\left(B_{r}, B_{R}\right) \leq 2\left(\int_{r}^{R} \frac{(s-r) d s}{V_{s}-V_{r}}\right)^{-1}
$$

(see [54] or [24, Theorem 7.1] - note that the proof of (5.8) uses the fact that $|\nabla d| \leq 1$, which is the case by (5.1)). By (5.8) and (5.4), we obtain

$$
\operatorname{cap}_{\mathcal{E}}\left(B_{r}, B_{2 r}\right) \leq 2\left(\int_{r}^{2 r} \frac{(s-r) d s}{M s^{2}}\right)^{-1}=2 M\left(\log 2-\frac{1}{2}\right)^{-1}<11 M,
$$

which was claimed.

Given (5.7), we see that all hypotheses of Theorem 4.17 are satisfied, so that (5.5) follows from (4.32). We need only to mention that (4.32) and (5.7) yield the coefficient 110 in (5.5), while we prefer 100, for the obvious esthetic reason. However, as one can see from the proof of Theorems 4.1 and 4.17, the constant factor 10 in (4.32) can be replaced by any number $>8$, for example, by 9 , which is enough to achieve the factor 100 in (5.5).

5.2. Riemann surfaces. Denote by $\Sigma_{\gamma}$ a closed orientable Riemann surface of genus $\gamma$.

Theorem 5.4. Let $g$ be a Riemannian metric on $\Sigma_{\gamma}$. Let $\mu$ be the Riemannian measure on the Riemannian manifold $X=\left(\Sigma_{\gamma}, g\right)$, and $\mathcal{E}=\mathcal{E}_{\mu}$ be the Riemannian energy form on $X$. Then, for any signed Radon measure $\sigma$ on $X$ such that $\sigma_{+}$is non-atomic,

$$
\operatorname{Neg}(\mathcal{E}-\sigma) \geq \frac{\sigma_{\delta}(X)}{C(\gamma+1)},
$$

where $C>0$ and $0<\delta<1$ are absolute constants.

Consequently, we obtain from (5.10) by Lemma 4.4 that for any non-atomic Radon measure $\nu$ on $X$ such that $0<\nu(X)<\infty$, and for any real $\lambda$, we have

$$
\mathcal{N}_{\lambda}(\mathcal{E}-\sigma, \nu) \geq \frac{\sigma_{\delta^{2}}(X)+\delta \lambda \nu(X)}{C(\gamma+1)}
$$

and, for any $k=1,2, \ldots$,

$$
\lambda_{k}(\mathcal{E}-\sigma, \nu) \leq \frac{C(\gamma+1)(k-1)-\sigma_{\delta^{2}}(X)}{\delta \nu(X)} .
$$

Remark 5.5. Already the case $\sigma=0, \nu=\mu$ of (5.12) is highly non-trivial. In this case (5.12) becomes

$$
\lambda_{k}(-\Delta) \leq C^{\prime}(\gamma+1) \frac{k-1}{\mu(X)}
$$

where $\Delta$ is the Laplace-Beltrami operator of $X$ (which is a generator of $\mathcal{E}$ ) and $C^{\prime}=C / \delta$. It is not difficult to prove that if $X$ is a connected compact $n$-dimensional Riemannian manifold then

$$
\lambda_{k}(-\Delta) \leq C_{X}\left(\frac{k-1}{\mu(X)}\right)^{2 / n} .
$$

However, the constant $C_{X}$ in (5.14), as it is suggested by the notation, depends on various geometric properties of $X$ (cf. Theorem 5.9 below) whereas the constant 
$C^{\prime}$ in (5.13) is universal, and only the genus $\gamma$ reflects the geometry (or rather the topology) of $X$ in (5.13). The estimate (5.13) for $k=2$ was first proved by Hersch [29] in the case $\gamma=0$ and by Yang and Yau [56] for any $\gamma$. For $k>2$ it was conjectured by Yau [57] and was eventually proved by Korevaar [37]. It was shown by Colbois and Dodziuk [11] that in the case $n>2$ one cannot have (5.14) with a universal constant $C$ instead of $C_{X}$.

Proof. The Riemannian metric $g$ determines a conformal class of $\Sigma_{\gamma}$. A wellknown consequence of the Riemann-Roch theorem says that the Riemann surface $\Sigma_{\gamma}$ (with a fixed conformal class) admits a non-constant meromorphic function of the topological degree at most $D:=\gamma+1$. Hence, there exists a conformal mapping $T: X \rightarrow \mathbb{S}^{2}$ of the topological degree $\leq D$ (see for example [56]).

Here we consider $\mathbb{S}^{2}$ as a Riemannian manifold with the canonical Riemannian metric. Let $d^{\prime}$ be the geodesic distance on $\mathbb{S}^{2}, \mu^{\prime}$ the Riemannian measure on $\mathbb{S}^{2}$, and $\mathcal{E}^{\prime}=\mathcal{E}_{\mu^{\prime}}$ be the Riemannian energy form on $\mathbb{S}^{2}$. Since the conformal mapping of two-dimensional Riemannian manifolds locally preserves the Riemannian energy form and the mapping $T$ has topological degree $\leq D$, we see that $T$ has the energy degree at most $D$, in the sense of Definition 2.11. Hence, by Lemma 2.12, we have

$$
N e g(\mathcal{E}-\sigma) \geq N e g\left(D \mathcal{E}^{\prime}-\sigma^{\prime}\right)=N e g\left(\mathcal{E}^{\prime}-D^{-1} \sigma^{\prime}\right),
$$

where $\sigma^{\prime}(\cdot):=\sigma\left(T^{-1}(\cdot)\right)$.

Obviously, $\left(\mathbb{S}^{2}, d^{\prime}\right)$ admits $(2, N)$-covering property with an absolute constant $N$, all balls on $\mathbb{S}^{2}$ are precompact, and, for any ball $B(x, r)$ on $\mathbb{S}^{2}$,

$$
\mu^{\prime}(B(x, r)) \leq \pi r^{2} .
$$

Applying Theorem 5.3 to the Riemannian metric space $\left(\mathbb{S}^{2}, d^{\prime}\right)$ and a signed measure $D^{-1} \sigma^{\prime}$ (clearly, $\sigma_{+}^{\prime}$ is non-atomic) we conclude

$$
N e g\left(\mathcal{E}^{\prime}-D^{-1} \sigma^{\prime}\right) \geq \frac{D^{-1} \sigma_{\delta}^{\prime}\left(\mathbb{S}^{2}\right)}{100 \pi}=\frac{\sigma_{\delta}(X)}{C D},
$$

where $C=100 \pi$ and $\delta \in(0,1)$ is an absolute constant. Combining with (5.15) we obtain (5.10).

The estimate (5.10) admits the following extension.

Corollary 5.6. Let $g$ be a Riemannian metric on $\Sigma_{\gamma}$ and let $X$ be a Riemannian manifold conformal to $\left(\Sigma_{\gamma} \backslash P, g\right)$ where $P$ is a finite subset of $\Sigma_{\gamma}$. Let $\mathcal{E}$ be the Riemannian energy form on $X$. Then, for any signed Radon measure $\sigma$ on $X$ such that $\sigma_{+}$is non-atomic, we have

$$
N e g(\mathcal{E}-\sigma) \geq \frac{\sigma_{\delta}(X)}{C(\gamma+1)}
$$

where $\delta$ and $C$ are the same as in Theorem 5.4.

Proof. Consider the manifold $X^{\prime}=\left(\Sigma_{\gamma}, g\right)$ and let $\mathcal{E}^{\prime}$ be the Riemannian energy form on $X^{\prime}$ with the domain $\mathcal{F}^{\prime}=\operatorname{Lip}_{0}\left(X^{\prime}\right)$. The conformal mapping identifies $X$ with $X^{\prime} \backslash P$. Set $\mathcal{F}=\operatorname{Lip}_{0}(X)$ and observe that $\mathcal{F} \subset \mathcal{F}^{\prime}$ and $\mathcal{E}[f]=$ $\mathcal{E}^{\prime}[f]$ for any $f \in \mathcal{F}$, because the Riemannian metric of $X$ and the metric $g$ are conformal.

Let $\sigma^{\prime}$ be the extension of the measure $\sigma$ to $X^{\prime}$ by setting $\left.\sigma^{\prime}\right|_{P}=0$. Then $\sigma^{\prime}$ is a signed Radon measure on $X^{\prime}$ such that $\sigma_{+}^{\prime}$ is non-atomic. Since $\left|\sigma^{\prime}\right|(P)=0$ and 
$\operatorname{cap}_{\mathcal{E}^{\prime}}(P, U)=0$ for any open set $U \subset X^{\prime}$ containing $P$, Lemma 2.13 and Theorem 5.4 yield

$$
N e g(\mathcal{E}-\sigma)=N e g\left(\mathcal{E}^{\prime}-\sigma^{\prime}\right) \geq \frac{\sigma_{\delta}\left(X^{\prime}\right)}{C(\gamma+1)}=\frac{\sigma_{\delta}(X)}{C(\gamma+1)}
$$

Example 5.7. Let $X=\left(\Sigma_{\gamma}, g\right)$ and let $K=K(x)$ be the Gauss curvature of the metric $g$ on $X$. Fix a real constant $\alpha$ and define a signed measure $\sigma$ on $X$ by $d \sigma=-\alpha K d \mu$ where $\mu$ is the the Riemannian measure on $X$. The energy form $(\mathcal{E}-\sigma, \mathcal{F})$ (where $\left.\mathcal{F}=\operatorname{Lip}_{0}(X)\right)$ is closable in $L^{2}(X, \mu)$ and its generator

$$
H=-\Delta+\alpha K
$$

has a discrete spectrum that can be estimated by (5.12) as follows. Observe that, by the Gauss-Bonnet formula,

$$
\sigma(X)=-\alpha \int_{X} K d \mu=-2 \pi \chi \alpha,
$$

where $\chi=2-2 \gamma$. Hence, Theorem 5.4 yields the following estimates, for all $k=1,2, \ldots$ :

If $\alpha K(x) \geq 0$ for all $x \in X$ (and hence $\sigma \leq 0$ and $\sigma_{\delta^{2}}=\sigma$ ) then

$$
\lambda_{k}(H) \leq \frac{C(\gamma+1)(k-1)+2 \pi \chi \alpha}{\delta \mu(X)} .
$$

If $\alpha K(x) \leq 0$ all $x \in X$ (and hence $\sigma \geq 0$ and $\sigma_{\delta^{2}}=\delta^{2} \sigma$ ) then

$$
\lambda_{k}(H) \leq \frac{C(\gamma+1)(k-1)+2 \delta^{2} \pi \chi \alpha}{\delta \mu(X)} .
$$

Example 5.8. Let $X=\left(\Sigma_{\gamma} \backslash P, g\right)$ where card $P=\kappa$, and $H$ be as above a generator of the energy form $(\mathcal{E}-\sigma, \mathcal{F})$ in $L^{2}(X, \mu)$. In this case (5.17) still holds but with the Euler characteristic $\chi=2-2 \gamma-\kappa$. Let $K(x) \leq 0$ on $X$, and $K \not \equiv 0$. Then, for any $\alpha>0$, we have $\sigma \geq 0$, and Corollary 5.6 yields

$$
N e g(H) \geq \frac{\delta \sigma(X)}{C(\gamma+1)}=\frac{2 \delta \pi(\kappa+2 \gamma-2) \alpha}{C(\gamma+1)} .
$$

By hypotheses we have $\chi<0$ and hence $\kappa+2 \gamma \geq 3$, which implies

$$
\kappa+2 \gamma-2 \geq \frac{1}{2}(\gamma+1) .
$$

Indeed, if (5.19) fails then $2 \kappa+3 \gamma \leq 4$, which is not compatible with $\kappa+2 \gamma \geq 3$. Substituting (5.19) into (5.18) we obtain that

$$
N e g(H) \geq \frac{\delta \pi}{C} \alpha=c \alpha,
$$

where $c$ is an absolute positive constant.

\subsection{Manifolds of higher dimension.}

Theorem 5.9. Let $\left(X, g_{0}\right)$ be a Riemannian manifold of dimension $n \geq 2, \mu_{0}$ be its Riemannian measure, and $d$ be a Riemannian pseudometric on $X$. Assume that that the following properties are satisfied, for some positive constants $M, N$ :

(a) space $(X, d)$ satisfies $(2, N)$-covering property;

(b) all balls in $(X, d)$ are precompact; 
(c) for any $r>0$ and for any $d$-ball $B_{r}$ of radius $r$ on $X$,

$$
\mu_{0}\left(B_{r}\right) \leq M r^{n} .
$$

Let $g$ be another metric on $X$, which is conformal to $g_{0}, \mu$ be the Riemannian measure of $g$, and $\mathcal{E}=\mathcal{E}_{\mu}$ be the Riemannian energy form of $g$. If $\mu(X)<\infty$ then, for any non-atomic Radon measure $\sigma$ on $X$,

$$
N e g(\mathcal{E}-\sigma) \geq\left\lfloor c \frac{\sigma(X)^{n / 2}}{\mu(X)^{n / 2-1}}\right\rfloor,
$$

where $c=c(n, N, M)>0$.

Remark 5.10. Applying (5.22) to $\lambda \sigma$ instead of $\sigma$, we obtain, for any $\lambda \geq 0$,

$$
\mathcal{N}_{\lambda}(\mathcal{E}, \sigma)=N e g(\mathcal{E}-\lambda \sigma) \geq\left\lfloor c \frac{\sigma(X)^{n / 2}}{\mu(X)^{n / 2-1}} \lambda^{n / 2}\right\rfloor .
$$

Similarly to Lemma 2.8, one obtains from (5.23) that, for all $k=1,2, \ldots$,

$$
\lambda_{k}(\mathcal{E}, \sigma) \leq C \frac{\mu(X)^{1-2 / n}}{\sigma(X)} k^{2 / n}
$$

where $C=C(n, N, M)$.

Remark 5.11. Theorem 5.9 is to some extent a higher order generalization of Theorem 5.3. Indeed, assuming that in Theorem $5.9 n=2$ and $g=g_{0}$, and that in Theorem $5.3 \mu$ is the Riemannian measure, we obtain the same statements. However, in general Theorem 5.3 is not reduced to Theorem 5.9 because in the former the measure $\mu$ does not have to be Riemannian, and the measure $\sigma$ can be signed.

Example 5.12. Let $\left(X, g_{0}\right)$ be a compact connected $n$-manifold and $d$ be the geodesic distance on $X$. Then the hypotheses $(a),(b),(c)$ are automatically satisfied with the constants $N, M$ depending on the metric $g_{0}$. The estimate (5.22) of $N e g\left(\mathcal{E}_{\mu}-\sigma\right)$ depends on the measures $\mu$ and $\sigma$ only via their total mass, provided $\mu$ is the Riemannian measure of a metric $g$ that is conformal to $g_{0}$. The constant $c$ in (5.22) depends on the metric $g$ only via its conformal class.

In the compact case the floor function in (5.22) can be dropped, that is the following is true:

$$
\operatorname{Neg}(\mathcal{E}-\sigma) \geq c \frac{\sigma(X)^{n / 2}}{\mu(X)^{n / 2-1}} .
$$

Indeed, if $\sigma(X)>0$ then the function $\varphi \equiv 1 \in \operatorname{Lip}_{0}(X)$ satisfies $\mathcal{E}[\varphi]<\sigma[\varphi]$ so that $\operatorname{Neg}(\mathcal{E}-\sigma) \geq 1$. Combining with (5.22) we obtain (5.25).

Note that in the case of a compact manifold and $\sigma=\mu$, the estimate (5.24) was first proved by Korevaar [37].

Example 5.13. Let $X=\mathbb{R}^{n}, g_{0}$ be the standard Euclidean metric, and $d$ be the Euclidean distance. Then all the hypotheses $(a),(b),(c)$ are satisfied. Let $a(x)$ be a smooth positive function on $\mathbb{R}^{n}, n>2$, and set $g=a g_{0}$ so that $d \mu=a^{n / 2} d \mu_{0}$. Let measure $\sigma$ be defined by $d \sigma=b d \mu_{0}$, where $b(x)$ is a continuous positive function on $\mathbb{R}^{n}$. Then the following operator

$$
L=\frac{1}{b} \operatorname{div}\left(a^{n / 2-1} \nabla\right)
$$


is a generator of the form $\mathcal{E}_{\mu}$ in $L^{2}(X, \sigma)$ (where $\nabla$ and div are related to $g_{0}$ ). Hence, (5.24) yields

$$
\lambda_{k}(L) \leq C \frac{\left(\int_{\mathbb{R}^{n}} a^{n / 2} d \mu_{0}\right)^{1-2 / n}}{\int_{\mathbb{R}^{n}} b d \mu_{0}} k^{2 / n},
$$

provided the both integrals are finite.

Proof of Theorem 5.9. Recall that for any capacitor $(F, G)$ on $(X, g)$, the capacity associated with the energy form $\mathcal{E}$ is defined by

$$
\operatorname{cap}(F, G)=\inf _{\mathcal{T}} \int_{X}\left|\nabla_{g} \varphi\right|^{2} d \mu,
$$

where $\mathcal{T}=\mathcal{T}(F, G)$ is the class of test functions, and $\nabla_{g}$ is the gradient associated with the metric $g$. Consider also the $n$-capacity defined by

$$
\operatorname{cap}^{(n)}(F, G)=\inf _{\mathcal{T}} \int_{X}\left|\nabla_{g} \varphi\right|^{n} d \mu .
$$

Since $n$ is the dimension of $X$, the $n$-capacity is preserved by a conformal change of the metric, that is

$$
\operatorname{cap}^{(n)}(F, G)=\inf _{\mathcal{T}} \int_{X}\left|\nabla_{g_{0}} \varphi\right|^{n} d \mu_{0} .
$$

In the metric $g_{0}$, the $n$-capacity of the capacitor $\left(B_{r}, R_{R}\right)$ (where $0<r<R$ and the balls $B_{r}, B_{R}$ are concentric) admits the following estimate

$$
\operatorname{cap}^{(n)}\left(B_{r}, R_{R}\right) \leq C\left(\int_{r}^{R}\left(\frac{s}{\mu_{0}\left(B_{s}\right)}\right)^{\frac{1}{n-1}} d s\right)^{1-n},
$$

(see $[\mathbf{1 4}],[\mathbf{3 2}])$, whence by $(5.21)$

$$
\operatorname{cap}^{(n)}\left(B_{r}, B_{2 r}\right) \leq C M
$$

(here $C$ denotes any positive constant depending only on $n, N$, and the value of $C$ may be different at different occurrences).

By the Hölder inequality, we obtain, for any $\varphi \in \mathcal{T}(F, G)$,

$$
\int_{X}\left|\nabla_{g} \varphi\right|^{2} d \mu \leq\left(\int_{G}\left|\nabla_{g} \varphi\right|^{n} d \mu\right)^{2 / n} \mu(G)^{1-2 / n},
$$

whence it follows that

$$
\operatorname{cap}(F, G) \leq\left(\operatorname{cap}^{(n)}(F, G)\right)^{2 / n} \mu(G)^{1-2 / n} .
$$

Similarly to the proof of Theorem 4.1, we can assume in the sequel that $0<$ $\sigma(X)<\infty$. By Corollary 3.12, for any positive integer $k$, there exists a family $\left\{A_{i}\right\}_{i=1}^{2 k}$ of annuli in $(X, d)$ such that

$$
\sigma\left(A_{i}\right) \geq c \frac{\sigma(X)}{k} \text { for all } i=1,2, \ldots, 2 k,
$$

and the annuli $\left\{2 A_{i}\right\}_{i=1}^{2 k}$ are disjoint. It follows from (5.26) that

$$
\operatorname{cap}^{(n)}\left(A_{i}, 2 A_{i}\right) \leq C M
$$

(cf. the proof of Theorem 4.7), whence (5.27) implies

$$
\operatorname{cap}\left(A_{i}, 2 A_{i}\right) \leq C M^{2 / n} \mu\left(2 A_{i}\right)^{1-2 / n} .
$$


Since

$$
\sum_{i=1}^{2 k} \mu\left(2 A_{i}\right) \leq \mu(X)
$$

there exists at least $k$ sets $2 A_{i}$ such that

$$
\mu\left(2 A_{i}\right) \leq \frac{\mu(X)}{k} .
$$

Without loss of generality, we can assume that this is the case for $i=1,2, \ldots, k$, whence it follows that

$$
\operatorname{cap}\left(A_{i}, 2 A_{i}\right) \leq C M^{2 / n}\left(\frac{\mu(X)}{k}\right)^{1-2 / n} \text { for } i=1,2, \ldots, k .
$$

Assume for a moment that the following inequality is true:

$$
C M^{2 / n}\left(\frac{\mu(X)}{k}\right)^{1-2 / n} \leq \frac{c}{2} \frac{\sigma(X)}{k},
$$

which implies by (5.29) and (5.28)

$$
\operatorname{cap}\left(A_{i}, 2 A_{i}\right) \leq \frac{1}{2} \sigma\left(A_{i}\right) \text { for } i=1,2, \ldots, k .
$$

Then choosing nearly optimal test functions for the capacitors $\left(A_{i}, 2 A_{i}\right), i=$ $1,2, . ., k$, we obtain a $k$-dimensional subspace $\mathcal{V} \subset \operatorname{Lip}_{0}(X)$ such that $\mathcal{E}[\varphi]<\sigma[\varphi]$ for any $\varphi \in \mathcal{V} \backslash\{0\}$, whence $\operatorname{Neg}(\mathcal{E}-\sigma) \geq k$.

Finally, noticing that the inequality (5.30) holds for any $k$ such that

$$
k \leq \frac{c^{\prime}}{M} \frac{\sigma(X)^{n / 2}}{\mu(X)^{n / 2-1}},
$$

we obtain (5.22).

5.4. Boundary surfaces. Let $X$ be a Riemannian manifold. In this section, we use the notation

$$
\lambda_{k}(X)=\lambda_{k}\left(\mathcal{E}_{\mu}, \mu\right)
$$

where $\mu$ is the Riemannian measure on $X$ and $\mathcal{E}_{\mu}$ is the energy form given by (5.3) with the domain $\mathcal{F}=\operatorname{Lip}_{0}(X)$.

Recall that a Riemannian $M$ is called a Cartan-Hadamard manifold if $M$ is complete, non-compact, simply connected manifold of non-positive sectional curvature. In particular, $\mathbb{R}^{n}$ and $\mathbb{H}^{n}$ are Cartan-Hadamard manifolds.

Theorem 5.14. Let $\Omega$ be a bounded open set in a 3-dimensional CartanHadamard manifold $M$, and let the boundary $\Gamma$ of $\Omega$ be smooth so that $\Gamma$ is a compact oriented Riemannian 2-manifold. Let $\gamma$ be the genus of $\Gamma$. Then, for all positive integers $k, m$,

$$
\lambda_{k}(\Omega)^{3 / 2} \geq \frac{c}{(\gamma+1)^{3 / 2}} \lambda_{k+1}(\Gamma) \sqrt{\frac{\lambda_{m+1}(\Gamma)}{m}},
$$

where $c>0$ is an absolute constant.

In particular, for all $k=1,2, \ldots$,

$$
\lambda_{k}(\Omega) \geq \frac{c}{\gamma+1} \frac{\lambda_{k+1}(\Gamma)}{k^{1 / 3}} .
$$


Proof. By a theorem of Hoffman and Spruck [31], a Cartan-Hadamard 3manifold admits the following isoperimetric inequality:

$$
V(\Omega) \leq C A(\Gamma)^{3 / 2},
$$

where $V$ stands for the volume in $M, A$ is the area on $\Gamma$, and $C$ is an absolute constant. It follows from (5.33) that $\lambda_{k}(\Omega)$ admits the lower bound

$$
\lambda_{k}(\Omega) \geq c\left(\frac{k}{V(\Omega)}\right)^{2 / 3}
$$

(see for example $[\mathbf{1 0}],[\mathbf{4 1}],[\mathbf{2 2}]$ ), where $c>0$ is an absolute constant.

On the other hand, by (5.13) we have the following upper bound for $\lambda_{k+1}(\Gamma)$ :

$$
\lambda_{k+1}(\Gamma) \leq C(\gamma+1) \frac{k}{A(\Gamma)},
$$

with an absolute constant $C$. Combining (5.35) and (5.33) we obtain

$$
\lambda_{k+1}(\Gamma) \sqrt{\frac{\lambda_{m+1}(\Gamma)}{m}} \leq\left(\frac{C(\gamma+1)}{A(\Gamma)}\right)^{3 / 2} k \leq \frac{C^{5 / 2}(\gamma+1)^{3 / 2} k}{V(\Omega)},
$$

which together with (5.34) implies (5.31). Clearly, (5.31) implies (5.32) for $m=k$.

5.5. Positive definite perturbations. The purpose of this section is to present a partial result towards the conjecture that the constant $\delta$ in Theorem 4.1 can be taken to be 1 .

Theorem 5.15. Let $X$ be a Riemannian manifold, $d$ be a pseudometric on $X$, $\mu$ be a Radon measure on $X$, and $\mathcal{E}=\mathcal{E}_{\mu}$. Assume that the following conditions hold, for some positive constants $N, Q$ :

(i) $(X, d)$ satisfies $(2, N)$-covering property;

(ii) measure $\mu$ is $d$-non-atomic and $0<\mu(X)<\infty$;

(iii) for any $d$-ball $B$ in $X$,

$$
\operatorname{cap}_{\mathcal{E}}(B, 2 B) \leq Q
$$

Let $\sigma$ be a finite signed Radon measure on $X$ such that the form $(\mathcal{E}-\sigma, \mathcal{F})$ is positive definite (where $\mathcal{F}=\operatorname{Lip}_{0}(X)$ ). Then, for any $\lambda \geq 0$,

$$
\mathcal{N}_{\lambda}(\mathcal{E}-\sigma, \mu) \geq \frac{\sigma(X)+\varepsilon \lambda \mu(X)}{10 Q},
$$

where $0<\varepsilon<1$ is a constant depending only on $N$. Also, for any $k=1,2, \ldots$,

$$
\lambda_{k}(\mathcal{E}-\sigma, \mu) \leq \frac{10 Q(k-1)-\sigma(X)}{\varepsilon \mu(X)} .
$$

Proof. By Lemma 4.18, if $\sigma(X)>0$ then $N e g(\mathcal{E}-\sigma) \geq 1$ which contradicts the hypothesis that $\mathcal{E}-\sigma$ is positive definite. Therefore, $\sigma(X) \leq 0$. Assuming that $\varepsilon^{-1} \sigma(X)+\lambda \mu(X)>0$ (otherwise, (5.36) is trivial), we obtain $\sigma(X)+\lambda \mu(X)>0$, whence by Lemma 4.18,

$$
\mathcal{N}_{\lambda}(\mathcal{E}-\sigma, \mu)=\operatorname{Neg}(\mathcal{E}-(\sigma+\lambda \mu)) \geq 1
$$

We will show that there exist $k$ functions $f_{i} \in \mathcal{F}$ with disjoint supports such that

$$
(\mathcal{E}-\sigma)\left[f_{i}\right]<\lambda \mu\left[f_{i}\right]
$$


where

$$
k:=\left\lfloor\frac{\sigma(X)+\varepsilon \lambda \mu(X)}{5 Q}\right\rfloor
$$

This will imply

$$
\mathcal{N}_{\lambda}(\mathcal{E}-\sigma, \mu) \geq k
$$

which together with (5.38) yields

$$
\mathcal{N}_{\lambda}(\mathcal{E}-\sigma, \mu) \geq \max (1, k) \geq \frac{\sigma(X)+\varepsilon \lambda \mu(X)}{10 Q},
$$

thus finishing the proof of (5.36). Clearly, the estimate (5.37) follows from (5.36) by Lemma 2.8 .

To prove the above claim observe that, by Corollary 4.9 , there exist $2 k$ disjoint capacitors $\left(F_{i}, G_{i}\right)$ on $X$ such that

$$
\mu\left(F_{i}\right) \geq c \frac{\mu(X)}{2 k} \text { and } \operatorname{cap}_{\mathcal{E}}\left(F_{i}, G_{i}\right) \leq 4 Q,
$$

where $c \in(0,1)$ depends only on $N$. Choose a test function $2 f_{i} \in \mathcal{T}\left(F_{i}, G_{i}\right)$ such that $\mathcal{E}\left[2 f_{i}\right]<5 Q$. Recall that $2 f_{i} \in \mathcal{T}\left(F_{i}, G_{i}\right)$ implies

$$
f \in C_{0}\left(G_{i}\right),\left.f\right|_{F_{i}}=\frac{1}{2}, 0 \leq f_{i} \leq \frac{1}{2} .
$$

Hence, we have, for $\varepsilon:=c / 8$,

$$
\mu\left[f_{i}\right] \geq \varepsilon \frac{\mu(X)}{k} \quad \text { and } \quad \mathcal{E}\left[f_{i}\right]<\frac{5}{4} Q
$$

which, in particular, implies

$$
\sum_{i=1}^{2 k} \mathcal{E}\left[f_{i}\right]<\frac{5}{2} Q k
$$

Let us prove that

$$
\sigma(X)-\sum_{i=1}^{2 k} \sigma\left[f_{i}\right] \leq \sum_{i=1}^{2 k} \mathcal{E}\left[f_{i}\right] .
$$

Assume for the moment that (5.43) has been proved. Then (5.42) and (5.43) imply

$$
\sigma(X)+\sum_{i=1}^{2 k}(\mathcal{E}-\sigma)\left[f_{i}\right]<\frac{5}{2} Q k+\sum_{i=1}^{2 k} \mathcal{E}\left[f_{i}\right] \leq 5 Q k
$$

and, hence,

$$
\sum_{i=1}^{2 k}(\mathcal{E}-\sigma)\left[f_{i}\right] \leq 5 Q k-\sigma(X) .
$$

Since $(\mathcal{E}-\sigma)\left[f_{i}\right] \geq 0$, there exists at least $k$ functions $f_{i}$ such that

$$
(\mathcal{E}-\sigma)\left[f_{i}\right]<\frac{5 Q k-\sigma(X)}{k}
$$

By (5.40) we have

$$
5 Q k \leq \sigma(X)+\varepsilon \lambda \mu(X),
$$

whence by (5.41)

$$
\frac{5 Q k-\sigma(X)}{k} \leq \lambda \mu\left[f_{i}\right]
$$


Together with (5.44), this yields (5.39).

We are left to prove (5.43). Define a function $h \geq 0$ on $X$ by the identity

$$
h^{2}+\sum_{i} f_{i}^{2}=1
$$

Since the supports of $f_{i}$ are disjoint and $0 \leq f_{i} \leq 1 / 2$, we obtain $h>1 / 2$. Integrating (5.45) against $\sigma$, we obtain

$$
\sigma(X)-\sum_{i} \sigma\left[f_{i}\right]=\sigma[h] .
$$

Since the form $\mathcal{E}-\sigma$ is positive definite and $h>1 / 2$, we obtain

$$
\sigma[h] \leq \mathcal{E}[h] \leq 2 \inf |h| \mathcal{E}[h] \leq \mathcal{E}\left[h^{2}\right] .
$$

Next, it follows from (5.45) that

$$
\nabla\left(h^{2}\right)=-\sum_{i} \nabla\left(f_{i}^{2}\right)
$$

whence

$$
\mathcal{E}\left[h^{2}\right]=\sum_{i} \mathcal{E}\left[f_{i}^{2}\right]
$$

Using

we obtain

$$
\mathcal{E}\left[f_{i}^{2}\right] \leq 2 \sup \left|f_{i}\right| \mathcal{E}\left[f_{i}\right] \leq \mathcal{E}\left[f_{i}\right]
$$

Combining (5.46), (5.47), (5.48), and (5.49) we obtain (5.43).

\section{Eigenvalues of the Jacobi operator}

Throughout this section, except for Subsection $6.3, X$ will be an oriented twodimensional manifold immersed into a three dimensional Riemannian manifold $M$. For simplicity of notation, we will not distinguish between the points of $X$ and their images in $M$ (although some points in $X$ may merge in $M$ ). We assume that $X$ is endowed with the induced Riemannian metric, and denote by $\mu$ the Riemannian measure on $X$. Let $K$ be the Gauss curvature of $X, R_{M}$ be the scalar curvature of $M$, and $\operatorname{Ric}_{M}$ be the Ricci curvature of $M$. Let $\mathbf{n}$ be an orthonormal vector field on $X$ in $M$.

Let $A$ be the operator of the second fundamental form of $X$, that is, at any point $x \in X, A=A(x)$ is a linear operator in $T_{x} X$ acting by $A \xi=-\nabla_{\xi} \mathbf{n}$. Denote $\|A\|^{2}:=\operatorname{trace}\left(A A^{*}\right)$, set

$$
q:=\operatorname{Ric}_{M}(\mathbf{n}, \mathbf{n})+\|A\|^{2},
$$

and consider the energy form

$$
\mathcal{A}[f]:=\int_{X}\left(|\nabla f|^{2}-q f^{2}\right) d \mu
$$

with the domain $\mathcal{F}=\operatorname{Lip}_{0}(X)$. In other words, $\mathcal{A}=\mathcal{E}-\sigma$ where $\mathcal{E}$ is the Riemannian energy form on $X$ and $\sigma$ is a signed measure defined by $d \sigma=q d \mu$.

It is known that the energy form $\mathcal{A}$ determines the second variation of the area functional under the normal deformation of $X$ (see for example $[\mathbf{1 2}]$, $[\mathbf{3 9}$, 
Section 6], [47]), while the first variation is determined by the mean curvature. We will be concerned with estimates of the counting function of the energy form $\mathcal{A}$, in particular, $N e g(\mathcal{A})$. If in addition $X$ is a minimal surface (that is, the mean curvature of $X$ vanishes everywhere) then the number $N e g(\mathcal{A})$ is called the stability index of $X$ and is denoted by $\operatorname{ind}(X)$. The minimal surface $X$ is called stable if ind $(X)=0$.

A generator of the form $(\mathcal{A}, \mathcal{F})$ in $L^{2}(X, \mu)$ is the following operator

$$
L:=-\Delta-q=-\Delta-\left(\operatorname{Ric}_{M}(\mathbf{n}, \mathbf{n})+\|A\|^{2}\right),
$$

which is called the stability operator or the Jacobi operator.

6.1. Riemann surfaces. Let $X$ and $M$ be as above.

Theorem 6.1. Assume that Ric $_{M} \geq 0$ and let $X$ be conformally equivalent to $\left(\Sigma_{\gamma} \backslash P, g\right)$, where $g$ is a Riemannian metric on $\Sigma_{\gamma}$ and $P$ is a finite subset of $\Sigma_{\gamma}$. Then

$$
N e g(\mathcal{A}) \geq \frac{c_{0}}{\gamma+1}\left(\int_{X} R_{M} d \mu-\int_{X} K d \mu\right)
$$

where $c_{0}$ is an absolute positive constant.

If, in addition, $\mu(X)<\infty$ then, for any $k=1,2, \ldots$,

$$
\lambda_{k}(\mathcal{A}, \mu) \leq \frac{C(\gamma+1) k}{\mu(X)}-\frac{c}{\mu(X)} \int_{X} R_{M} d \mu
$$

where $C$ and $c$ are absolute positive constants.

Remark 6.2. Recall that by the Gauss-Bonnet formula

$$
\int_{X} K d \mu=2 \pi \chi
$$

where $\chi$ is the Euler characteristic of $X$. In the present setting we have

$$
\chi=2-2 \gamma-\kappa,
$$

where $\kappa:=\operatorname{card} P$.

Remark 6.3. In the case when $X$ is compact we have by Theorem 5.4 that

$$
\lambda_{k}(\mathcal{E}, \mu) \leq \frac{C(\gamma+1) k}{\mu(X)} .
$$

The additional non-negative term $\int_{X} R_{M} d \mu$ in (6.3) reflects the distinction between the Jacobi operator and the Laplace operator.

Proof. We use the following identity on $X$ :

$$
R_{M}-\operatorname{Ric}_{M}(\mathbf{n}, \mathbf{n})=K+\frac{\|A\|^{2}}{2}-\frac{|H|^{2}}{2},
$$

where $H$ is the mean curvature vector of $X$. It implies

$$
q=\operatorname{Ric}_{M}(\mathbf{n}, \mathbf{n})+\|A\|^{2}=R_{M}-K+\frac{\|A\|^{2}}{2}+\frac{|H|^{2}}{2} \geq R_{M}-K,
$$

whence

$$
\sigma(X)=\int_{X} q d \mu \geq \int_{X} R_{M} d \mu-\int_{X} K d \mu=\int_{X} R_{M} d \mu-2 \pi \chi
$$


By $\operatorname{Ric}_{M} \geq 0$ and (6.1) we have $q \geq 0$ and hence $\sigma \geq 0$. In particular, $\sigma_{\delta}=\delta \sigma$, and by Corollary 5.6 we conclude

$$
N e g(\mathcal{A})=N e g(\mathcal{E}-\sigma) \geq \frac{\delta \sigma(X)}{C(\gamma+1)}
$$

whence (6.2) follows with $c_{0}=\delta / C$.

The second claim follows from (5.12) and (6.6) using also $\chi \leq 2-2 \gamma$ :

$$
\begin{aligned}
\lambda_{k}(\mathcal{A}, \mu) & \leq \frac{C(\gamma+1)(k-1)-\delta^{2} \sigma(X)}{\delta \mu(X)} \\
& \leq \frac{C(\gamma+1) k-\delta^{2} \int_{X} R_{M} d \mu-\left[C(\gamma+1)+\delta^{2} 2 \pi(2 \gamma-2)\right]}{\delta \mu(X)} \\
& \leq \frac{C}{\delta} \frac{(\gamma+1) k}{\mu(X)}-\frac{\delta}{\mu(X)} \int_{X} R_{M} d \mu,
\end{aligned}
$$

provided $C \geq 4 \pi \delta^{2}$, which can be assumed to be true. Renaming the constants we obtain (6.3).

Remark 6.4. The hypothesis $\operatorname{Ric}_{M} \geq 0$ is only needed to conclude that $q \geq 0$. One can also obtain $q \geq 0$ using different assumptions. For example, it is true provided $R_{M} \geq 0$ and $K \leq 0$, as one can see from (6.5).

Theorem 6.1 may have many applications. For example, (6.2) and (6.4) imply the following statement.

Corollary 6.5. Under the hypotheses of Theorem 6.1 , if in addition $R_{\min }:=$ $\inf _{X} R_{M}>0$ and $X$ is immersed in $M$ as a minimal surface then

$$
\mu(X) \leq \frac{C_{0}(\gamma+1) \text { ind }(X)+2 \pi \chi}{R_{\min }},
$$

where $C_{0}$ is an absolute positive constant.

6.2. Minimal surfaces in $\mathbb{R}^{3}$ with finite total curvature. In this section, we assume by default that $X$ is an oriented immersed minimal surface in $\mathbb{R}^{3}$. Then we have $\|A\|^{2}=-2 K$ and, hence, the second variation form $\mathcal{A}$ is given by $\mathcal{A}=\mathcal{E}-\sigma$ where $\sigma$ is defined by

$$
d \sigma=-2 K d \mu .
$$

In particular, we have $\sigma \geq 0$ and

$$
\sigma(X)=2 K_{\text {total }}(X)
$$

where

$$
K_{\text {total }}(X):=\int_{X}|K| d \mu .
$$

The first result related ind $(X)$ to the total curvature is due to Barbosa and do Carmo [1] who proved that

$$
K_{\text {total }}(X)<2 \pi \quad \Longrightarrow \quad \text { ind }(X)=0 .
$$

A number of authors [5], [18], [49] independently proved the following extension of Bernstein's theorem: the only complete stable minimal surface is a plane. In other words, if $X$ is complete then

$$
K_{\text {total }}(X) \equiv 0 \quad \Longleftrightarrow \quad \text { ind }(X)=0 .
$$


Fischer-Colbrie [17] proved that if $X$ is complete then

$$
K_{\text {total }}(X)<\infty \Longleftrightarrow \text { ind }(X)<\infty .
$$

These results suggests that there may exist inequalities relating $\operatorname{ind}(X)$ and $K_{\text {total }}(X)$. Indeed, it was proved by Tysk [55] that if $X$ is compete then

$$
\operatorname{ind}(X) \leq c_{0} K_{\text {total }}(X)
$$

where $c_{0} \approx 0.6133$. Two of the authors proved in $[\mathbf{2 8}]$ that $(6.7)$ holds for any (not necessarily complete) minimal surface $X$, although with a very large constant $c_{0}$.

For a complete $X$ the estimate (6.7) was improved by Micallef [44]: if $X$ is complete and not a plane then

$$
\operatorname{ind}(X) \leq \frac{1}{\pi} K_{\text {total }}(X)+2 \gamma-3,
$$

where $\gamma$ is the genus of $X$. If in addition $K_{\text {total }}<\infty$ and all branching values of the extended Gauss map of $X$ lie on an equator of $\mathbb{S}^{2}$, then by a theorem of Montiel and Ros $[45]$,

$$
\text { ind }(X)=\frac{1}{2 \pi} K_{\text {total }}(X)-1 \text {. }
$$

It was conjectured in [7] and [30] that if $X$ is complete and non-planar then

$$
\text { ind }(X) \leq \frac{1}{2 \pi} K_{\text {total }}(X)-1 \text {. }
$$

The known examples of complete oriented minimal surfaces suggest that ind $(X)$ may admit also a lower bound via $K_{\text {total }}(X)$. We prove here some weak versions of this conjecture. Before we do so, let us briefly recall some results about the structure of minimal surfaces in $\mathbb{R}^{3}$. We refer a reader to the surveys by Hoffman and Karcher [30] and by Meeks and Perez [43] for more details.

Let $X$ be a complete minimal surface. Since we are interested in lower estimates of ind $(X)$, we can assume that ind $(X)<\infty$ and hence $K_{\text {total }}(X)<\infty$. Then, by a theorem of Huber [33] (see also [48]) $X$ is conformally equivalent to $\Sigma_{\gamma} \backslash P$ where $P=\left\{p_{1}, \ldots, p_{k}\right\}$ is a finite subset of $\Sigma_{\gamma}$. Moreover, a punctured neighborhood in $\Sigma_{\gamma}$ of each point $p_{i}$ corresponds to an end $E_{i}$ of $X$.

Let $n(x)$ be a normal unit vector field on $X$ in $\mathbb{R}^{3}$. When a point $x \in E_{i}$ escapes to $\infty$ along $E_{i}$ then $n(x)$ has a limit, say $n_{i}$. Denote by $C_{i}$ the large circle on the unit sphere $\mathbb{S}^{2}$ such that the plane through $C_{i}$ has the normal $n_{i}$.

For any $r>0$, consider the set

$$
C(r):=\mathbb{S}^{2} \cap \frac{1}{r} X
$$

where $\frac{1}{r} X$ is the scaling transformation of $X$ in $\mathbb{R}^{3}$. By a theorem of Jorge and Meeks [35], for large enough $r$, the set $C(r)$ consists of $k$ immersed closed curves on $\mathbb{S}^{2}$, say $C_{1}(r), \ldots, C_{k}(r)$ (assuming that the ordering of $\gamma_{r}^{i}$ matches that of $E_{i}$ ), and when $r \rightarrow \infty$, the curve $C_{i}(r)$ converges in $C^{\infty}$-sense to the circle $C_{i}$, with a multiplicity $m_{i}$, where $m_{i}$ is a positive integer (see Fig. 11).

In particular, the length of the circle $C_{i}$ is $2 \pi m_{i}$. We say that the end $E_{i}$ has multiplicity $m_{i}$. It is known that $m_{i}=1$ if and only if the end $E_{i}$ is embedded.

Theorem 6.6. Let $X$ be a complete oriented minimal surface immersed in $\mathbb{R}^{3}$. If ind $(X)<\infty$ then

$$
\operatorname{ind}(X) \geq \frac{c}{m} K_{\text {total }}(X)
$$




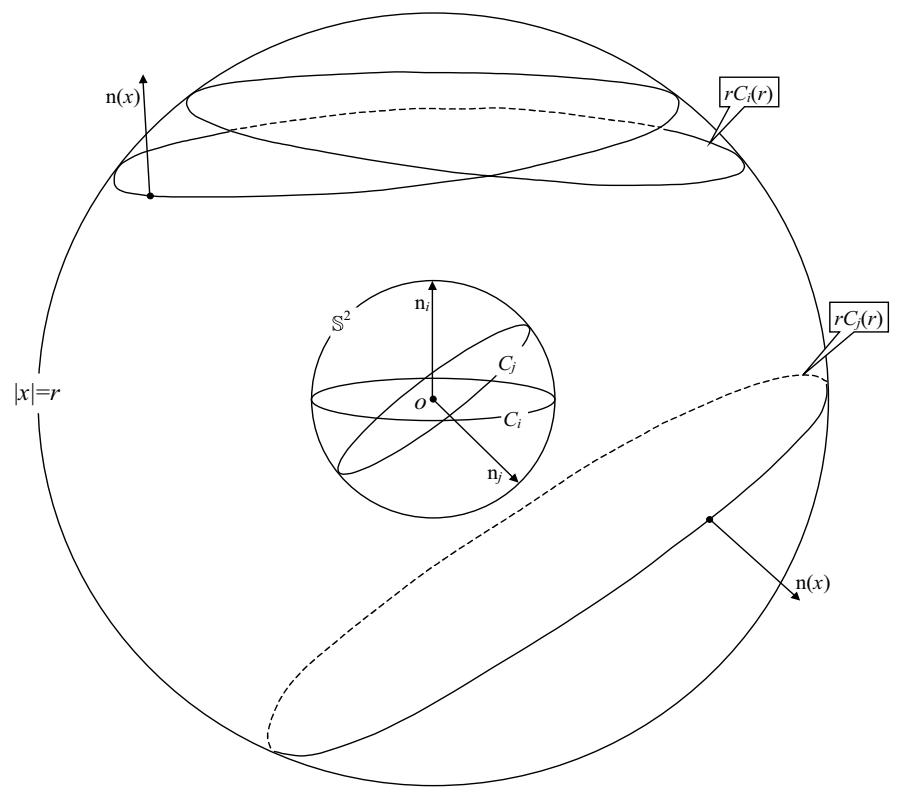

Figure 11. Circle $C_{i}$ with multiplicity 2 and circle $C_{j}$ with multiplicity 1.

where $m=m_{1}+\ldots+m_{k}$ is the total multiplicity of the ends of $X$, and $c$ is an absolute positive constant.

If in addition the ends of $X$ are embedded then

$$
\operatorname{ind}(X) \geq \frac{c}{k} K_{\text {total }}(X),
$$

where $k$ is the number of ends of $X$.

Proof. Let $d_{0}$ be the geodesic distance on $X$ with respect to the induced metric. Denote by $d$ the extrinsic distance on $X$, that is the restriction to $X$ of the Euclidean distance in $\mathbb{R}^{3}$. Then $(X, d)$ is a Riemannian pseudometric space (see Section 5). Let $\mu$ be the Riemannian measure on $X$ and $\mathcal{E}$ be the Riemannian energy form on $X$. Let us show that the hypotheses $(a)$ - $(c)$ of Theorem 5.3 are satisfied.

Let $B(x, r)$ be a $d$-ball on $X$, that is $B(x, r)$ is the intersection of the Euclidean ball $\widetilde{B}(x, r)$ in $\mathbb{R}^{3}$ with $X$. The ball $\widetilde{B}(x, r)$ can be covered by at most $N$ euclidean balls in $\mathbb{R}^{3}$ of radii $r / 4$, where $N$ is an absolute constant. Select out of them those balls that have non-empty intersection with $X$, and let their centers be $y_{1}, y_{2}, \ldots, y_{k}$, where $k \leq N$. Let $x_{i}$ be a point in the intersection of $\widetilde{B}\left(y_{i}, r / 4\right)$ with $X$. Then $\widetilde{B}\left(x_{i}, r / 2\right)$ covers $\widetilde{B}\left(y_{i}, r / 4\right)$ whence it follows that all balls $B\left(x_{i}, r / 2\right)$ cover $B(x, r)$. Hence, $(X, d)$ satisfies $(2, N)$-covering property, that is the hypothesis $(a)$ holds.

Since $X$ is complete and $K_{\text {total }}(X)<\infty$, the immersion of $X$ into $\mathbb{R}^{3}$ is proper, that is the intersection of any compact set in $\mathbb{R}^{3}$ with $X$ is compact in the topology of $X$ (see [43, Section 2.3]). This immediately implies that $d$-balls in $X$ are precompact, that is the hypothesis $(b)$. 
Let us prove that, for any $d$-ball $B(x, r)$ in $X$,

$$
\mu(B(x, r)) \leq \pi m r^{2},
$$

which will settle the hypothesis $(c)$. It is a consequence of the minimality of $X$ that the function

$$
r \mapsto \frac{\mu(B(x, r))}{r^{2}}
$$

is increasing (see [53, p.84]). Therefore, it suffices to prove (6.10) asymptotically, that is

$$
\mu(B(x, r)) \sim \pi m r^{2} \quad \text { as } r \rightarrow \infty,
$$

for any fixed $x \in X$. Without loss of generality, we will prove this for $x=o$, where $o$ is the origin of $\mathbb{R}^{3}$.

Set $S(r)=\partial B(o, r), \rho(x)=|x|\left(\right.$ where $\left.x \in \mathbb{R}^{3}\right)$ and observe that by the coarea formula,

$$
\mu(B(o, R))=\int_{0}^{R}\left(\int_{S(r)}|\nabla \rho|^{-1} d l\right) d r,
$$

where $\nabla$ is the Riemannian gradient on $X$ and $d l$ is the length element on $S(r)$. Let $\widetilde{\nabla}$ be the Euclidean gradient in $\mathbb{R}^{3}$. Then $\nabla \rho(x)$ is the projection of $\widetilde{\nabla} \rho(x)$ onto $T_{x} X$ (see Fig. (12)) and since $n(x)$ is a normal to $T_{x} X$, we obtain

$$
|\nabla \rho|^{2}=|\widetilde{\nabla} \rho|^{2}-(\widetilde{\nabla} \rho \cdot n)^{2}=1-\left(\frac{x}{\rho} \cdot n\right)^{2} .
$$

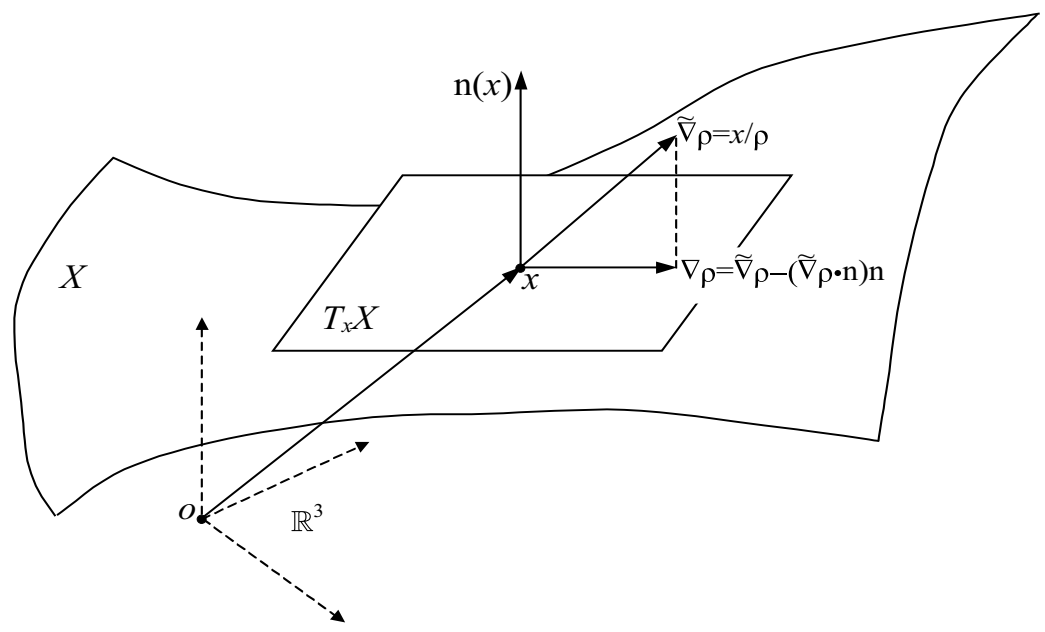

FiguRE 12. Gradients $\nabla \rho$ and $\widetilde{\nabla} \rho$

If $x \rightarrow \infty$ along the end $E_{i}$, then $x / \rho \in C_{i}(\rho)$ and hence $x / \rho$ tends on $\mathbb{S}^{2}$ to the circle $C_{i}$ whereas $n(x)$ tends to $n_{i}$. Since $n_{i}$ is orthogonal to $C_{i}$, we obtain

$$
\frac{x}{\rho} \cdot n \longrightarrow 0
$$


and hence $|\nabla \rho| \rightarrow 1$. For large enough $r, S(r)$ is the union of the curves $r C_{i}(r)$. Therefore, we obtain that, for $r \rightarrow \infty$,

$$
\int_{S(r)}|\nabla \rho|^{-1} d l \sim l(S(r))=\sum_{i=1}^{k} l\left(r C_{i}(r)\right) \sim \sum_{i=1}^{k} l\left(C_{i}\right) r=2 \pi m r,
$$

whence by $(6.12)$

$$
\mu(B(o, R)) \sim \int_{0}^{R} 2 \pi m r d r=\pi m R^{2} .
$$

This finishes the proof of (6.11) and hence (6.10).

Finally, we claim that measure $\sigma$ on $X$ (given by $d \sigma=-2 K d \mu$ ) is $d$-nonatomic. Let $I$ be the immersion in question of the manifold $X$ into $\mathbb{R}^{3}$. It follows from the definition of the extrinsic distance $d$ that, for any $x \in X$,

$$
\{y \in X: d(x, y)=0\}=I^{-1}(x) .
$$

By the definition of an immersion, for any point $y \in X$ there is an open neighbourhood $U$ of $y$ in $X$ such that $\left.I\right|_{U}$ is an injection. Therefore, $I^{-1}(x)$ consists of isolated points and hence $\sigma\left(I^{-1}(x)\right)=0$, that is $\sigma$ is $d$-non-atomic.

Applying Theorem 5.3 we obtain

$$
\text { ind }(X)=N e g(\mathcal{E}-\sigma) \geq \frac{\delta \sigma(X)}{100 \pi m}=\frac{c}{m} K_{\text {total }}(X),
$$

where $c=\frac{\delta}{50 \pi}$ is an absolute positive constant. In the case when the ends of $X$ are embedded, we have $m=k$, whence (6.9) follows.

Note that by Corollary 5.6 we have also in the above setting that

$$
\text { ind }(X)=N e g(\mathcal{E}-\sigma) \geq \frac{\sigma_{\delta}(X)}{C(\gamma+1)}=\frac{c^{\prime}}{\gamma+1} K_{\text {total }}(X),
$$

where $c^{\prime}=2 \sigma / C$. However, in most applications (6.9) gives a better lower bound for ind $(X)$ than (6.13).

Theorem 6.7. Let $X$ be a connected complete oriented minimal surface embedded in $\mathbb{R}^{3}$. If ind $(X)<\infty$ then

$$
\operatorname{ind}(X) \geq k-1
$$

where $k$ is the number of ends of $X$. niques.

This theorem will be proved Section 6.4 after introducing the necessary tech-

Corollary 6.8. For any connected complete oriented minimal surface $X$ embedded in $\mathbb{R}^{3}$, we have

$$
\operatorname{ind}(X) \geq c^{\prime} \sqrt{K_{\text {total }}(X)}
$$

and

$$
\text { ind }(X) \geq c^{\prime \prime} \sqrt{\text { genus }(X)},
$$

where $c^{\prime}, c^{\prime \prime}$ are absolute positive constants. 
Proof. If ind $(X)=\infty$ then there is nothing to prove, so assume ind $(X)<\infty$ and hence $K_{\text {total }}(X)<\infty$. Let $k$ be the number of ends of $X$. If $k=1$ then $(6.15)$ follows from (6.9) and the fact that ind $(X)$ is an integer. If $k \geq 2$ then $(6.9)$ and (6.14) imply

$$
\operatorname{ind}(X) \geq \frac{1}{2}\left(\frac{c}{k} K_{\text {total }}+\frac{k}{2}\right) \geq c^{\prime} \sqrt{K_{\text {total }}}
$$

where $c^{\prime}=\frac{1}{2} \sqrt{c / 2}$.

To prove (6.16) observe that, by a theorem of Osserman (see also [35]), we have

$$
K_{\text {total }}(X)=4 \pi(\gamma+k-1) \geq 4 \pi \gamma,
$$

where $\gamma=$ genus $(X)$. Hence, (6.16) follows from (6.15) with $c^{\prime \prime}=c^{\prime} \sqrt{4 \pi}$.

Let us mention for comparison the following result of Jorge and Meeks [35]: there exists a function $F:[0,+\infty) \rightarrow[0,+\infty)$ such that if $M$ is a properly embedded minimal surface in $\mathbb{R}^{3}$ then

$$
\text { ind }(X) \leq F(\text { genus }(X)) \text {. }
$$

Here no assumption is made about finiteness of the total curvature.

6.3. Counting functions of subsets. In this section, we assume that $X$ is a Riemannian manifold, $d$ is a Riemannian pseudometric on $X$, and $\mu$ is a Radon measure on $X$ having a continuous positive density with respect to the Riemannian measure. Let $\mathcal{E}=\mathcal{E}_{\mu}$ be the associated energy form with the domain $\mathcal{F}=\operatorname{Lip}_{0}(X)$. As was already mentioned, the form $(\mathcal{E}, \mathcal{F})$ is closable in $L^{2}(X, \mu)$, and its generator is $-\Delta_{\mu}$.

Let $\sigma$ be another Radon measure on $X$ defined by

$$
d \sigma=q d \mu
$$

where $q$ is a positive continuous function on $X$. The operator $-\frac{1}{q} \Delta_{\mu}$ is a generator of the form $(\mathcal{E}, \mathcal{F})$ in $L^{2}(X, \sigma)$. For any open set $\Omega \subset X$, consider the form $\mathcal{E}$ with the domain $\mathcal{F}(\Omega):=\mathcal{F} \cap C_{0}(\Omega)=\operatorname{Lip}_{0}(\Omega)$. The form $(\mathcal{E}, \mathcal{F}(\Omega))$ is closable in $L^{2}(\Omega, \sigma)$. Let $H_{\Omega}$ be its self-adjoint generator, and $\widetilde{\mathcal{F}}(\Omega)$ be the domain of the closure. Set

$$
N(\Omega)=\sup \{\operatorname{dim} \mathcal{V}: \mathcal{V} \prec \mathcal{F}(\Omega) \quad \text { and } \quad \mathcal{E}[f]<\sigma[f] \quad \forall f \in \mathcal{V} \backslash\{0\}\}
$$

and

$$
N^{*}(\Omega)=\sup \{\operatorname{dim} \mathcal{V}: \mathcal{V} \prec \widetilde{\mathcal{F}}(\Omega) \quad \text { and } \quad \mathcal{E}[f] \leq \sigma[f] \quad \forall f \in \mathcal{V}\} .
$$

By Lemma 2.7, we have

$$
N(\Omega)=\operatorname{dim} \operatorname{Im} \mathbf{1}_{(-\infty, 1)}\left(H_{\Omega}\right)
$$

and

$$
N^{*}(\Omega)=\operatorname{dim} \operatorname{Im} \mathbf{1}_{(-\infty, 1]}\left(H_{\Omega}\right) .
$$

Lemma 6.9. Let $(X, \mu)$ be a connected weighted manifold, and let $\Omega_{0}, \Omega_{1}, \ldots, \Omega_{n}$ be non-empty disjoint open sets in $X$. Then

$$
N(X) \geq N\left(\Omega_{0}\right)+\sum_{i=1}^{n} N^{*}\left(\Omega_{i}\right) .
$$


Proof. The proof follows an argument of Montiel and Ros [45]. Set $\mathcal{V}=$ $\operatorname{Im} \mathbf{1}_{(-\infty, 1)}(H)$ where $H$ is the self-adjoint generator of the form $(\mathcal{E}, \mathcal{F})$ in $L^{2}(X, \sigma)$. Let also $\mathcal{V}_{i}$ be finite dimensional linear spaces such that

$$
\mathcal{V}_{0} \subset \operatorname{Im} \mathbf{1}_{(-\infty, 1)}\left(H_{\Omega_{0}}\right)
$$

and

$$
\mathcal{V}_{i} \subset \operatorname{Im} \mathbf{1}_{(-\infty, 1]}\left(H_{\Omega_{i}}\right) \quad \text { for } i=1,2, \ldots, n .
$$

Since $\Omega_{i}$ are disjoint sets, the spaces $\mathcal{V}_{i}$ are all mutually orthogonal in $L^{2}(X, \sigma)$.

To prove (6.17), it suffices to show that

$$
\operatorname{dim} \mathcal{V} \geq \operatorname{dim} \mathcal{V}_{0}+\sum_{i=1}^{n} \operatorname{dim} \mathcal{V}_{i}=: m
$$

Assume from the contrary that $\operatorname{dim} \mathcal{V}<m$. Then there exists a non-zero function $v \in \bigoplus_{i=0}^{n} \mathcal{V}_{i}$ such that $v$ is orthogonal to $\mathcal{V}$ in $L^{2}(X, \sigma)$. Therefore,

$$
v=\int_{[1,+\infty)} d E_{t}(v)
$$

where $\left\{E_{t}\right\}_{t \in \mathbb{R}}$ is the spectral resolution of the operator $H$. Similarly to (2.26) we have

$$
\sigma[v]=\int_{[1,+\infty)} d\left\|E_{t} v\right\|^{2} \quad \text { and } \quad \mathcal{E}[v]=\int_{[1,+\infty)} t d\left\|E_{t} v\right\|^{2}
$$

whence

$$
\mathcal{E}[v] \geq \sigma[v] .
$$

On the other hand, for any $f \in \mathcal{V}_{i}$ we have $\mathcal{E}[f] \leq \sigma[f]$. Since $v$ is a linear combination of functions from $\mathcal{V}_{i} \subset \mathcal{F}\left(\Omega_{i}\right)$ and the sets $\Omega_{i}$ are disjoint, we obtain

$$
\mathcal{E}[v] \leq \sigma[v] .
$$

Hence, $\mathcal{E}[v]=\sigma[v]$ which is only possible if the measure $d\left\|E_{t} v\right\|^{2}$ does not charge $(1,+\infty)$, that is

$$
v=\int_{\{1\}} d E_{t}(v)
$$

so that $v$ is an eigenfunction of $H$ with the eigenvalue 1 . In particular, $v$ satisfies on $X$ the elliptic equation

$$
\Delta_{\mu} v+q v=0 .
$$

On the other hand, since for any $f \in \mathcal{V}_{0} \backslash\{0\}$ we have

$$
\mathcal{E}[f]<\sigma[f],
$$

the projection of $v$ onto $\mathcal{V}_{0}$ must vanish (otherwise, we would get $\mathcal{E}[v]<\sigma[v]$ ). This means that $v \in \bigoplus_{i=1}^{n} \mathcal{V}_{i}$ and hence $v \equiv 0$ in $\Omega_{0}$. Since $X$ is connected, the well-known property of solutions to the elliptic equations yields that $v \equiv 0$ in $X$, which contradicts the construction of $v$.

Definition 6.10. A weighted manifold $(X, \mu)$ is called parabolic if $\operatorname{cap}_{\mathcal{E}}(K, X)=$ 0 for any compact set $K \subset X$. 
Lemma 6.11. A weighted manifold $(X, \mu)$ is parabolic provided anyone of the following conditions is satisfied:

(a) There exists a constant $Q$ such that for any ball $B$ in $X$,

$$
\operatorname{cap}_{\mathcal{E}}(B, 2 B) \leq Q \text {. }
$$

(b) All balls of pseudometric $d$ are precompact and there exists a constant $C$ such that, for any ball $B(x, r)$,

$$
\mu(B(x, r)) \leq C r^{2} .
$$

Proof. Assume that $(a)$ holds. Any compact set in $X$ is bounded and hence is covered by a ball. Therefore, it suffices to show that $\operatorname{cap}_{\mathcal{E}}(B, X)=0$ for any ball $B$. It was shown in the proof of Lemma 4.18 that for balls $B_{n}=B\left(x, 2^{n}\right)$ the following inequality holds:

$$
\operatorname{cap}_{\mathcal{E}}\left(B_{n}, B_{m}\right) \leq \frac{Q}{m-n}
$$

where $m>n$ are positive integers (see (4.37). Letting $m \rightarrow \infty$ we obtain $\operatorname{cap}_{\mathcal{E}}\left(B_{n}, X\right)=$ 0 , which settles the claim.

The fact that $(b)$ implies the parabolicity of $X$ was essentially proved in [6] (see also [21], [24], [54]). Alternatively, one can use that $(b) \Longrightarrow(a)$, which was shown in the proof of Theorem 5.3 (cf. inequality (5.9)).

Definition 6.12. A non-empty open set $\Omega \subset X$ is called $q$-massive if there exists a function $u \in C^{2}(\Omega) \cap C(\bar{\Omega})$ such that

$$
0 \leq u \leq 1, \quad\left(\Delta_{\mu}+q\right) u \geq 0 \text { in } \Omega,\left.\quad u\right|_{\partial \Omega}=0, \quad u \not \equiv 0 .
$$

Lemma 6.13. Let the weighted manifold $(X, \mu)$ be geodesically complete and parabolic. If $\Omega \subset X$ is a q-massive open set and $\sigma(\Omega)<\infty$ then $N^{*}(\Omega) \geq 1$.

Proof. We shall prove that $u \in \widetilde{\mathcal{F}}(\Omega)$ and $\mathcal{E}[u] \leq \sigma[u]$, which will imply $N^{*}(\Omega) \geq 1$. Fix a smooth non-negative function $\eta: \mathbb{R} \rightarrow \mathbb{R}$ such that $0 \leq \eta^{\prime} \leq 1$ and $\left.\eta\right|_{(-\infty, \varepsilon]} \equiv 0$, for some $\varepsilon>0$ (see Fig. 13).

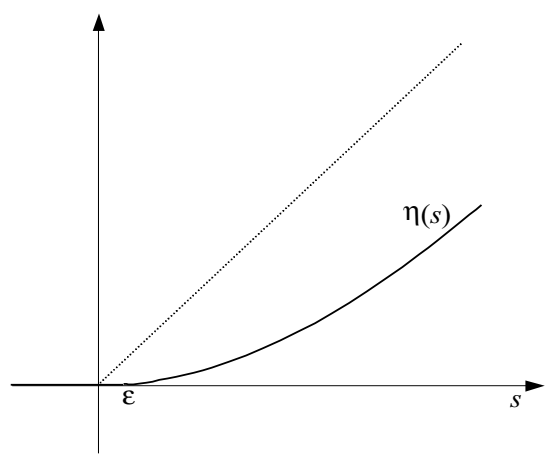

Figure 13. A function $\eta(s)$

Set $v=\eta(u)$ and observe that the function $v$ vanishes in a neighborhood of $\partial \Omega$. For any non-negative function $\varphi \in C_{0}^{\infty}(X)$, multiplying the inequality

$$
\Delta_{\mu} u+q u \geq 0
$$


by $v \varphi^{2}$ and integrating by parts in $\Omega$, we obtain

$$
\int_{\Omega} \nabla u \cdot \nabla v \varphi^{2} d \mu+2 \int_{\Omega} \nabla u \cdot \nabla \varphi v \varphi d \mu \leq \int_{\Omega} q u v \varphi^{2} d \mu .
$$

Let us allow the parameter $\varepsilon$ in the definition of function $\eta$ to vary and to tend to 0 so that we have a functional family $\left\{\eta_{\varepsilon}\right\}_{\varepsilon>0}$ and respectively the family $v_{\varepsilon}=\eta_{\varepsilon}(u)$. The function $v_{\varepsilon} \varphi$ is clearly in the class $\mathcal{F}(\Omega)=\operatorname{Lip}_{0}(\Omega)$, for any $\varepsilon>0$. Choose the family $\left\{\eta_{\varepsilon}\right\}$ so that

$$
\eta_{\varepsilon}^{\prime}(s) \rightarrow 1 \quad \text { as } \varepsilon \rightarrow 0, \text { for all } s>0 .
$$

In particular, $\eta_{\varepsilon}(s) \leq s$ and $\eta_{\varepsilon}(s) \rightarrow s$ as $\varepsilon \rightarrow 0$. This implies, by the dominated convergence theorem, that the following convergences take place, both in $L^{2}(\Omega, \mu)$ and $L^{2}(\Omega, \sigma)$ :

$$
v_{\varepsilon} \varphi \rightarrow u \varphi, \quad \varphi \nabla v_{\varepsilon} \rightarrow \varphi \nabla u, \quad \nabla\left(v_{\varepsilon} \varphi\right) \rightarrow \nabla(u \varphi) .
$$

In particular, it follows that $u \varphi \in \widetilde{\mathcal{F}}(\Omega)$. Setting $v=v_{\varepsilon}$ in $(6.23)$ and letting $\varepsilon \rightarrow 0$ we obtain

$$
\int_{\Omega}|\nabla u|^{2} \varphi^{2} d \mu+2 \int_{\Omega} \nabla u \cdot \nabla \varphi u \varphi d \mu \leq \int_{\Omega} q u^{2} \varphi^{2} d \mu .
$$

Adding $\int_{\Omega} u^{2}|\nabla \varphi|^{2} d \mu$ to the both sides, we obtain in the left hand side a complete square, that is

$$
\int_{\Omega}|\nabla(u \varphi)|^{2} d \mu \leq \int_{\Omega} q u^{2} \varphi^{2} d \mu+\int_{\Omega} u^{2}|\nabla \varphi|^{2} d \mu .
$$

Finally, using $|u| \leq 1$, we obtain

$$
\mathcal{E}[u \varphi] \leq \sigma[u \varphi]+\int_{\Omega}|\nabla \varphi|^{2} d \mu .
$$

Next, let us construct by induction a sequence of functions $\left\{\varphi_{n}\right\} \subset \mathcal{F}$ such that

$$
0 \leq \varphi_{n} \leq 1, \quad \varphi_{n} \leq \varphi_{n+1}, \quad \varphi_{n} \not \equiv 0, \quad \text { and } \mathcal{E}\left[\varphi_{n}\right]<1 / n .
$$

Indeed, fix a point $x \in X$ and set $B_{r}=B(x, r)$. Since $\operatorname{cap}_{\mathcal{E}}\left(B_{1}, X\right)=0$, we can choose a test function $\varphi_{1} \in \mathcal{T}\left(B_{1}, X\right)$ so that $\mathcal{E}\left[\varphi_{1}\right]<1$. Assuming that $\varphi_{n}$ is already constructed, find such a large number $r$ that $\operatorname{supp} \varphi_{n} \subset B_{r}$. Since $\operatorname{cap}_{\mathcal{E}}\left(B_{r}, X\right)=0$, we can choose a test function $\varphi_{n+1} \in \mathcal{T}\left(B_{n}, X\right)$ so that $\mathcal{E}\left[\varphi_{n+1}\right]<1 /(n+1)$. Finally, the monotonicity condition $\varphi_{n} \leq \varphi_{n+1}$ is satisfied because $\varphi_{n} \leq 1$ while $\varphi_{n+1}=1$ on $\operatorname{supp} \varphi_{n}$.

Setting $u_{n}=u \varphi_{n}$ and using (6.24), (6.25), we obtain

$$
\mathcal{E}\left[u_{n}\right] \leq \sigma\left[u_{n}\right]+\frac{1}{n}
$$

By construction, the sequence $\left\{u_{n}\right\}$ is monotone increasing and converges to $u$ pointwise. By the dominated convergence theorem, we obtain that $u_{n} \rightarrow u$ in $L^{2}(\Omega, \sigma)$. Let us prove that also $\mathcal{E}\left[u_{n}-u\right] \rightarrow 0$. Indeed, by the construction of the functions $\varphi_{n}$, we have

$$
\nabla u_{n}=\varphi_{n} \nabla u+u \nabla \varphi_{n} \longrightarrow \nabla u \quad \text { pointwise as } n \rightarrow \infty .
$$

By Fatou's lemma and (6.26), we obtain

$$
\mathcal{E}[u] \leq \liminf _{n \rightarrow \infty} \mathcal{E}\left[u_{n}\right] \leq \liminf _{n \rightarrow \infty} \sigma\left[u_{n}\right]=\sigma[u] \leq \sigma(\Omega) .
$$


On the other hand, we have

$$
\begin{aligned}
\mathcal{E}\left[u-u_{n}\right] & =\int_{\Omega}\left|\nabla\left(\left(1-\varphi_{n}\right) u\right)\right|^{2} d \mu \\
& \leq 2 \int_{\Omega}\left|\nabla \varphi_{n}\right|^{2} u^{2} d \mu+2 \int_{\Omega}\left(1-\varphi_{n}\right)^{2}|\nabla u|^{2} d \mu \\
& \leq \frac{2}{n}+2 \int_{\Omega}\left(1-\varphi_{n}\right)^{2}|\nabla u|^{2} d \mu .
\end{aligned}
$$

Since the sequence $\left\{1-\varphi_{n}\right\}_{n=1}^{\infty}$ is bounded and goes to 0 pointwise, while by (6.27) the measure $|\nabla u|^{2} d \mu$ is finite, we obtain by the dominated convergence theorem that $\mathcal{E}\left[u-u_{n}\right] \rightarrow 0$. Since $u_{n} \in \widetilde{\mathcal{F}}(\Omega)$, we conclude that also $u \in \widetilde{\mathcal{F}}(\Omega)$. By $(6.27)$ we have $\mathcal{E}[u] \leq \sigma[u]$, which finishes the proof.

Corollary 6.14. Let the weighted manifold $(X, \mu)$ be geodesically complete, connected, and parabolic, and let $\sigma(X)<\infty$. Assume that, for some positive integer $n$, there exist disjoint non-empty open sets $\Omega_{0}, \Omega_{1}, \ldots, \Omega_{n}$ in $X$ such that $\Omega_{i}$ are $q$-massive for all $i=1,2, \ldots, n$. Then

$$
N e g(\mathcal{E}-\sigma) \geq n
$$

Proof. Indeed, by Lemma 6.13 , we have, for any $i=1,2, \ldots, n$,

$$
N^{*}\left(\Omega_{i}\right) \geq 1
$$

and, by Lemma 6.9,

$$
N e g(\mathcal{E}-\sigma)=\mathcal{N}_{1}(\mathcal{E}, \sigma)=N(X) \geq \sum_{i=1}^{n} N^{*}\left(\Omega_{i}\right) \geq n .
$$

6.4. Lower bound of the stability index via the number of ends. Here we prove Theorem 6.7. We assume throughout that $X$ is a connected complete oriented minimal surface embedded into $\mathbb{R}^{3}$ such that ind $(X)<\infty$ and hence $K_{\text {total }}(X)<\infty$. As before, let $\mu$ be the Riemannian measure on $X$ and $\mathcal{E}$ be the Riemannian energy form on $X$. Set $q:=-2 K \geq 0$ and define a measure $\sigma$ on $X$ by $d \sigma=q d \mu$. We need to prove (6.14), that is

$$
N e g(\mathcal{E}-\sigma) \geq k-1,
$$

where $k$ is the number of ends of $X$.

Let $d$ be the extrinsic distance on $X$. It was shown in the proof of Theorem 6.6 that, for any $d$-ball $B(x, r)$ on $X$,

$$
\mu(B(x, r)) \leq \text { const } r^{2} .
$$

Therefore, by Lemma $6.11, X$ is parabolic. Hence, all the hypotheses of the first sentence of Corollary 6.14 are satisfied. Therefore, (6.30) will be proved if we construct $k$ disjoint non-empty open sets $\Omega_{1}, \ldots, \Omega_{k}$ on $X$ such that each $\Omega_{i}$ is $q$-massive ${ }^{7}$.

\footnotetext{
${ }^{7}$ For application of Corollary 6.14 , it suffices to show that $k-1$ sets out of the family $\left\{\Omega_{i}\right\}_{i=1}^{k}$ are $q$-massive. In our construction all $k$ sets $\Omega_{i}$ happen to be $q$-massive. However, this does not imply ind $(X) \geq k$ because the closures $\overline{\Omega_{i}}$ may cover all $X$ so that there may be no place for one more non-empty open set as it is required by Corollary 6.14 .
} 
Fix a normal unit vector field $n(x)$ on $X$, a unit vector $v \in \mathbb{R}^{3}$, and consider the following function on $X$

$$
u(x):=n(x) \cdot v,
$$

which is known to satisfy on $X$ the Jacobi equation

$$
\Delta u+q u=0 .
$$

Consider the open set

$$
\Omega:=\{x \in X: u(x) \neq 0\},
$$

and let $\Omega^{\prime}$ be a connected component of $\Omega$. Then either $u$ or $-u$ satisfies (6.22) in $\Omega^{\prime}$ so that $\Omega^{\prime}$ is $q$-massive. Hence, it suffices to show that, for an appropriate choice of the vector $v$, the set $\Omega$ has at least $k$ connected components.

For that we will use the additional information about the structure of the ends of $X$, which comes from the fact that $X$ is embedded. By a result of Schoen [52], after a rigid rotation of $X$ in $\mathbb{R}^{3}$, each end $E$ of $X$ can be represented (far enough from the origin) as the graph in $\mathbb{R}^{3}$ of the following function

$$
x_{3}=a+b \log r+\frac{c^{\prime} x_{1}+c^{\prime \prime} x_{2}}{r^{2}}+O\left(r^{-2}\right),
$$

where $r=\sqrt{x_{1}^{2}+x_{2}^{2}}$ and $a, b, c^{\prime}, c^{\prime \prime}$ are real constants. If $b=0$ then the end $E$ is asymptotic to the horizontal plane $x_{3}=a$, whereas in the case $b \neq 0$ the end $E$ is asymptotic to the catenoid

$$
r=2 \cosh \left(\frac{x_{3}-a}{b}\right) .
$$

In the former case, we refer to $E$ as a planar end, and in the latter case - as a catenoidal end (see Fig. 14).

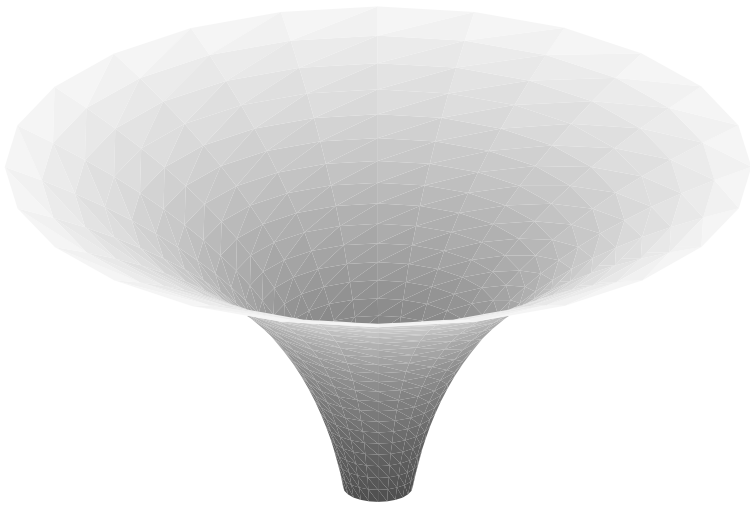

Figure 14. A catenoidal end.

All ends of $X$ are naturally ordered by the way they intersect a remote vertical line $l$. Namely, let $h(E)$ be the $x_{3}$-coordinate of the point where $E$ meets $l$ (see Fig. 15).

Definition 6.15. We say that the end $E$ is below the end $E^{\prime}$ if $h(E)<h\left(E^{\prime}\right)$. 


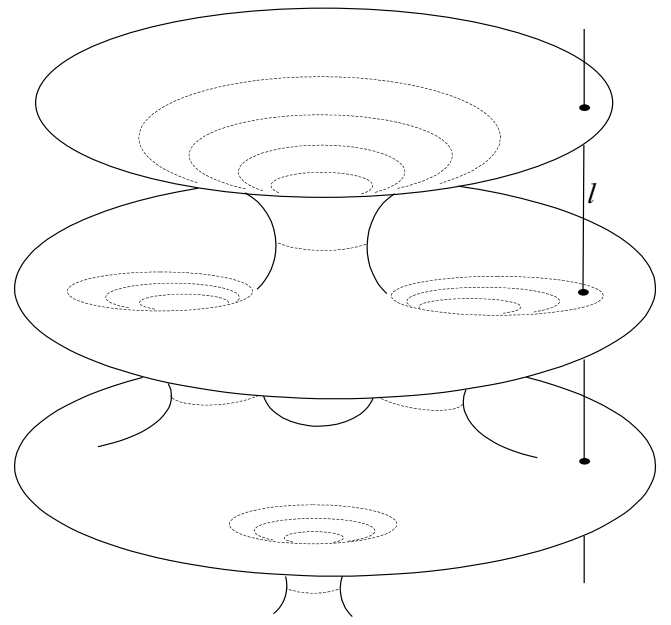

FIGURE 15. Ordering the ends of an embedded minimal surface according to their intersections with a vertical line $l$.

Clearly, this definition does not depend on the choice of $l$ provided the distance from $l$ to the origin $o$ is large enough (for a more general result on ordering of the ends of embedded minimal surfaces see [19]). We say that the ends $E$ and $E^{\prime}$ are neighbors if there is no end $E^{\prime \prime}$ between $E$ and $E^{\prime}$ in the sense of the order "below".

For any end $E$, the normal vector field $n(x)$ has the limit as $x$ goes to $\infty$ along $E$, so let $n(E)$ denote this limit. Clearly, $n(E)$ is vertical, that is $n(E)=(0,0,1)$ or $n(E)=(0,0,-1)$.

Lemma 6.16. If $E$ and $E^{\prime}$ are two ends of $X$, which are neighbors, then $n(E)=-n\left(E^{\prime}\right)$.

Proof. Let $x$ and $y$ be the points of intersection of respectively $E$ and $E^{\prime}$ with a remote vertical line $l$. Choose $l$ far enough so that $n(x)$ and $n(y)$ are "nearly" vertical and that the segment $[x, y]$ of $l$ does not intersect $X$ except for the points $x, y$. Since $X$ is connected, there is a path $\gamma:[0,1] \rightarrow X$ connecting $x$ and $y$ on $X$. Fix $\varepsilon>0$ and consider the deformed path in $\mathbb{R}^{3}$

$$
\gamma_{\varepsilon}(t)=\gamma(t)+\varepsilon n(\gamma(t)), \quad t \in[0,1] .
$$

The path $\gamma_{\varepsilon}$ connects in $\mathbb{R}^{3}$ the points $x_{\varepsilon}$ and $y_{\varepsilon}$ where

$$
x_{\varepsilon}=x+\varepsilon n(x) \text { and } y_{\varepsilon}=y+\varepsilon n(y) .
$$

If $\varepsilon$ is small enough then $\gamma_{\varepsilon}(t)$ does not intersect $X$ (see Fig. 16). Therefore, any other path from $x_{\varepsilon}$ to $y_{\varepsilon}$ must have even number of intersections with $X$.

Contrary to what we need to prove, assume that

$$
n(E)=n\left(E^{\prime}\right) \text {. }
$$

Then there is a path from $x_{\varepsilon}$ to $y_{\varepsilon}$ that crosses $X$ exactly once, at the point $y$ : this path is obtained by slightly modifying the path $\left[x_{\varepsilon}, x\right] \#[x, y] \#\left[y, y_{\varepsilon}\right]$ near the point $x$ so that it does not meet $X$ in a neighborhood of $x$. This contradiction finishes the proof. 


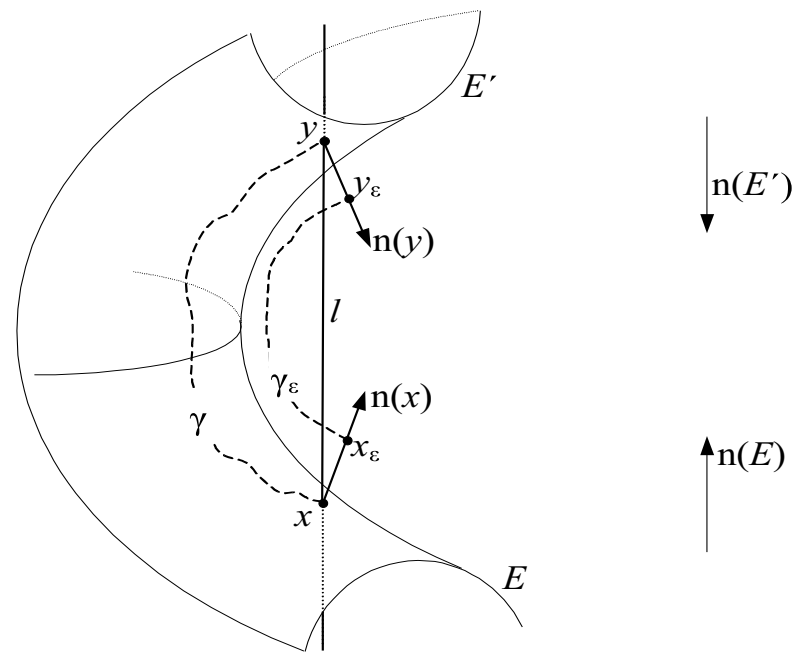

Figure 16. Paths $\gamma$ and $\gamma_{\varepsilon}$.

Choose the vector $v$ in (6.31) as follows: $v=(0,0,1)$. If $x \rightarrow \infty$ along an end $E$ then $u(x) \rightarrow n(E) \cdot v= \pm 1$. Let us say that an end $E$ is positive if $u(x) \rightarrow 1$ on $E$ and $E$ is negative of $u(x) \rightarrow-1$ on $E$. It follows from Lemma 6.16 that positive and negative ends alternate relative to the order "below". Let $E_{1}, E_{2}, \ldots, E_{k}$ be all ends of $X$ and let $\Omega_{i}$ be the connected component of the set $\Omega=\{u \neq 0\}$ containing a neighborhood of $\infty$ in $E_{i}$. Clearly, if the end $E_{i}$ is positive then $u>0$ in $\Omega_{i}$, and if $E_{i}$ is negative then $u<0$ in $\Omega_{i}$. This implies that the components $\Omega_{i}$ and $\Omega_{j}$, which correspond to neighboring ends $E_{i}$ and $E_{j}$, are disjoint. Therefore, all components $\Omega_{i}, i=1,2, \ldots, k$, are disjoint, which finishes the proof of Theorem 6.7.

Remark 6.17. The main point of the above proof was to show that the function $u=n(x) \cdot v$ has at least $k$ components of constant sign. Having proved that, we could have used instead of Corollary 6.14 a result of Choe [7] about a vision number, which says that if this particular function $u$ has $k$ components of constant sign then ind $(X) \geq k-1$. We have preferred a more general approach based on $q$-massive sets, because this approach does not need a function $u$ to be defined on the entire manifold $X$. This approach might work for immersed minimal surfaces where one can expect to be able to construct a function $u$ satisfying (6.22) separately for each end.

\section{References}

[1] Barbosa J.L., do Carmo M., On the size of a stable minimal surface in $\mathbb{R}^{3}$, Amer. J. Math., 98 (1976) no.2, 515-528.

[2] Barlow M.T., Diffusions on fractals, in: "Lectures on Probability Theory and Statistics, Ecole d'été de Probabilités de Saint-Flour XXV - 1995", Lecture Notes Math. 1690, Springer, 1998. 1-121.

[3] Barlow M.T., Heat kernels and sets with fractal structure, in: "Heat kernels and analysis on manifolds, graphs, and metric spaces", Contemporary Mathematics, 338 (2003) 11-40.

[4] Barlow M.T., Anomalous diffusion and stability of Harnack inequalities, in: "Surveys in Differential Geometry", 2004. 
[5] do Carmo M., Peng C.K., Stable minimal surfaces in $\mathbb{R}^{3}$ are planes, Bulletin of the AMS, 1 (1979) 903-906.

[6] Cheng S.Y., Yau S.-T., Differential equations on Riemannian manifolds and their geometric applications, Comm. Pure Appl. Math., 28 (1975) 333-354.

[7] Choe J., Index, vision number and stability of complete minimal surfaces, Arch. Rat. Mech. Anal., 109 (1990) no.3, 195-212.

[8] Chung F.R.K., Grigor'yan A., Yau S.-T., Upper bounds for eigenvalues of the discrete and continuous Laplace operators, Advances in Math., 117 (1996) 165-178.

[9] Chung F.R.K., Grigor'yan A., Yau S.-T., Eigenvalues and diameters for manifolds and graphs, in: "Tsing Hua Lectures on Geometry and Analysis", Ed. S.-T.Yau, International Press, 1997. 79-105.

[10] Chung F.R.K., Grigor'yan A., Yau S.-T., Higher eigenvalues and isoperimetric inequalities on Riemannian manifolds and graphs, Comm. Anal. Geom, 8 (2000) no.5, 969-1026.

[11] Colbois B., Dodziuk J., Riemannian metrics with large $\lambda_{1}$, Proceedings of AMS, 122 (1994) no.3, 905-906.

[12] Colding T.H., Minicozzi W.P. II, "Minimal surfaces", Courant Lecture Notes in Math.4, 1999.

[13] Colding T.H., Minicozzi W.P. II, An excursion into geometric analysis, in: "Surveys in Differential Geometry", 2004.

[14] Coulhon T., Holopainen I., Saloff-Coste L., Harnack inequality and hyperbolicity for subelliptic $p$-Laplacians with applications to Picard type theorems, Geom. Funct. Anal., 11 (2001)

[15] Davies E.B., "Heat kernels and spectral theory", Cambridge University Press, 1989.

[16] Davies E.B., "Spectral theory and differential operators", Cambridge University Press, 1995.

[17] Fischer-Colbrie D., On complete minimal surfaces with finite Morse index in three manifolds, Invent. Math., 82 (1985) 121-132.

[18] Fischer-Colbrie D., Schoen R., The structure of complete stable minimal surfaces in 3-manifolds of non-negative scalar curvature, Comm. Pure Appl. Math., 33 (1980) 199-211.

[19] Frohman C., Meeks W.H. III, The ordering theorem for the ends of properly embedded minimal surfaces, Topology, 36 (1997) no.3, 605-617.

[20] Fukushima M., Oshima Y., Takeda M., "Dirichlet forms and symmetric Markov processes", Studies in Mathematics 19, De Gruyter, 1994.

[21] Grigor'yan A., On the existence of positive fundamental solution of the Laplace equation on Riemannian manifolds, (in Russian) Matem. Sbornik, 128 (1985) no.3, 354-363. Engl. transl. Math. USSR Sb., 56 (1987) 349-358.

[22] Grigor'yan A., Heat kernel upper bounds on a complete non-compact manifold, Revista Matemática Iberoamericana, 10 (1994) no.2, 395-452.

[23] Grigor'yan A., Isoperimetric inequalities and capacities on Riemannian manifolds, Operator Theory: Advances and Applications, 109 (1999) 139-153.

[24] Grigor'yan A., Analytic and geometric background of recurrence and non-explosion of the Brownian motion on Riemannian manifolds, Bull. Amer. Math. Soc., 36 (1999) 135-249.

[25] Grigor'yan A., Heat kernels and function theory on metric measure spaces, in: "Heat kernels and analysis on manifolds, graphs, and metric spaces", Contemporary Mathematics, 338 (2003) 143-172.

[26] Grigor'yan A., Hu J., Lau K.S., Heat kernels on metric-measure spaces and an application to semi-linear elliptic equations, Trans. Amer. Math. Soc., 355 (2003) no.5, 2065-2095.

[27] Grigor'yan A., Yau S.-T., Decomposition of a metric space by capacitors, in: "Differential Equations: La Pietra 1996", Ed. Giaquinta et. al., Proceeding of Symposia in Pure Mathematics, 65 1999. 39-75.

[28] Grigor'yan A., Yau S.-T., Isoperimetric properties of higher eigenvalues of elliptic operator, Amer. J. Math, 125 (2003) 893-940.

[29] Hersch J., Quatre properiétés isopérimétriques de membranes sphériques homogènes, C.R. Acad. Sci. Paris, 270 (1970) 1645-1648.

[30] Hoffman D., Karcher H., Complete embedded minimal surfaces of finite total curvature, in: "Geometry, V", Encyclopaedia Math. Sci. 90, Springer, Berlin, 1997. 5-93, 267-272.

[31] Hoffman D., Spruck J., Sobolev and isoperimetric inequalities for Riemannian submanifolds, Comm. Pure Appl. Math., 27 (1974) 715-727. See also "A correction to: Sobolev 
and isoperimetric inequalities for Riemannian submanifolds", Comm. Pure Appl. Math., 28 (1975) no.6, 765-766.

[32] Holopainen I., Volume growth, Green's functions and parabolicity of ends, Duke Math. J., 97 (1999) no.2, 319-346.

[33] Huber A., On subharmonic functions and differential geometry in the large, Comment. Math. Helvetici, 32 (1957) 181-206.

[34] Jakobson D., Nadirashvili N., Polterovich I., Extremal metric for the first eigenvalue on a Klein bottle, to appear in Canad. J. Math

[35] Jorge L., Meeks W.H. III, The topology of complete minimal surfaces of finite total Gaussian curvature, Topology, 22 (1983) no.2, 203-221.

[36] Kigami J., "Analysis on fractals", Cambridge University Press, 2001.

[37] Korevaar N., Upper bounds for eigenvalues of conformal metric, J. Diff. Geom., 37 (1993) 73-93.

[38] Levin D., Solomyak M., The Rozenblum-Lieb-Cwikel inequality for Markov generators, J. d'Analyse Math., 71 (1997) 173-193.

[39] Li P., Treibergs A., Applications of eigenvalue techniques to geometry, in: "Contemporary Geometry", Univ. Ser. Math., Plenum, New York, 1991. 21-52.

[40] Li P., Yau S.-T., A new conformal invariant and its applications to the Willmore conjecture and the first eigenvalue of compact surfaces, Invent. Math., 69 (1982) 269-291.

[41] Li P., Yau S.-T., On the Schrödinger equation and the eigenvalue problem, Comm. Math. Phys., 88 (1983) 309-318.

[42] Maz'ya V.G., “Sobolev spaces”, (in Russian) Izdat. Leningrad Gos. Univ. Leningrad, 1985. Engl. transl. Springer, 1985.

[43] Meeks W. H. III, Pérez J., Conformal properties in classical minimal surface theory, in: "Surveys in Differential Geometry", 2004.

[44] Micallef M., Comparison of index of energy with index of area of minimal surfaces, in preparation

[45] Montiel S., Ros A., Schrödinger operators associated to a holomorphic map, in: "Global Differental Geometry and Global Analysis", Lecture Notes Math. 1481, Springer, 1990. 147174.

[46] Nadirashvili N., Berger's isoperimetric problem and minimal immersions of surfaces, Geom. Funct. Anal., 6 (1996) 877-897.

[47] Nitsche J.C.C., "Lectures on minimal surfaces, vol.1", Cambridge University Press, 1989.

[48] Osserman R., "A survey of minimal surfaces", Dover, New York, 1986.

[49] Pogorelov A.V., On the stability of minimal surfaces, Soviet Math. Dokl., 24 (1981) 274-276.

[50] Reed M., Simon B., "Methods of modern mathematical physics. II: Fourier analysis, self-adjointness", Academic Press, 1975.

[51] Saloff-Coste L., "Aspects of Sobolev inequalities", LMS Lecture Notes Series 289, Cambridge Univ. Press, 2002.

[52] Schoen R., Uniqueness, symmetry, and embeddedness of minimal surfaces, J. Diff. Geom., 18 (1983) 791-809.

[53] Simon L.M., "Lectures on geometric measure theory", Proceedings of the Centre for Mathematical Analysis, Australian National University, 3, Australian National University, Centre for Mathematical Analysis, Canberra, 1983.

[54] Sturm K-Th., Sharp estimates for capacities and applications to symmetrical diffusions, Probability theory and related fields, 103 (1995) no.1, 73-89.

[55] Tysk J., Eigenvalue estimates with applications to minimal surfaces, Pacific J. Math., 128 (1987) 361-366.

[56] Yang P., Yau S.-T., Eigenvalues of the Laplacian of compact Riemann surfaces and minimal submanifolds, Ann. Scuola Norm. Sup. Pisa Cl. Sci. (4), 7 (1980) 55-63.

[57] Yau S.-T., Survey on partial differential equations in differential geometry, Ann. Math. Studies, 102 (1982) 3-70. 
Imperial College London, London SW7 2AZ, United Kingdom

E-mail address: a.grigoryan@imperial.ac.uk

University of Bristol, University Walk, Bristol, BS8 1TW, United Kingdom

E-mail address: y.netrusov@bristol.ac.uk

Harvard University, CAMbridge MA 02138, USA

E-mail address: yau@math.harvard.edu 